\title{
A Pareto-efficient combustion framework with submodel assignment for predicting complex flame configurations
}

\author{
Hao $\mathrm{Wu}^{*}$, Yee Chee See, Qing Wang, Matthias Ihme \\ Department of Mechanical Engineering, Stanford University, Stanford, CA 94305
}

\begin{abstract}
The selection of an appropriate combustion model for the numerical prediction of reacting flows remains an outstanding issue. Often, expert knowledge or experimental data is required to make an informed decision in selecting a suitable model. Furthermore, the computational cost that is associated with the application of certain combustion models introduces another constraint in the selection process. By addressing these issues, the objective of this work is to develop a Pareto-efficient combustion (PEC) framework for application to complex chemically reacting flows under consideration of user-specific input about quantities of interest, desired simulation accuracy and computational cost, and a set of combustion models. PEC utilizes a Pareto efficiency, and introduces a manifold drift term as a measure for determining the adequacy of using a certain combustion-manifold model to predict selected quantities of interest. Since underlying model assumptions are encoded in the manifold, PEC restricts the application of submodels within its intended use. Further, the proposed approach for evaluating the manifold drift provides a rigorous method for combining different combustion models - as long as they can be described by a manifold. As such, this formulation represents a general description for the selection of combustion models, thereby overcoming potential limitations of flame-topology indicators and regime-specific combustion models. The capability of the PEC-framework is demonstrated in application to a tribrachial flame. By considering combustion models from the class of reaction-transport manifolds (inert mixing, equilibrium, flamelet/progress variable, and flame-prolongation in ILDM) and chemistry manifolds (using detailed and skeletal mechanisms), it is shown that PEC locally adapts the submodel fidelity within the user-defined threshold for selected quantities of interest. A parametric analysis is conducted to illustrate the dynamic range of the PEC-framework in accommodating Pareto-efficient submodel arrangements.
\end{abstract}

Keywords: Pareto-efficient combustion framework; Combustion modeling; Manifold; Turbulent combustion; Multi-regime combustion

\section{Contents}

\section{Introduction}

2 Governing equations and manifold models 5

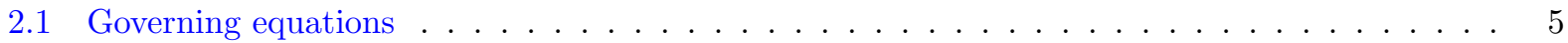

2.2 Manifold methods . . . . . . . . . . . . . . . . . . . . . . . . . 5

3 Pareto-efficient combustion modeling framework 6

3.1 Preliminaries . . . . . . . . . . . . . . . . . . . . . . 6

\footnotetext{
${ }^{*}$ Corresponding author

Email addresses: wuhao@stanford.edu (Hao Wu), seeyc@stanford.edu (Yee Chee See), wangqing@stanford.edu (Qing
} Wang), mihme@stanford.edu (Matthias Ihme) 
3.2 Pareto efficiency of combustion models . . . . . . . . . . . . . . . . . . 6

3.3 Computational cost and error estimation . . . . . . . . . . . . . . . . . . 8

3.3.1 Drift from manifold . . . . . . . . . . . . . . . . . . . 8

3.3.2 Cost function for model error . . . . . . . . . . . . . . . . . . . . . . 9

3.4 Coupling strategy at the interface of subdomains . . . . . . . . . . . . . . . . . 10

3.4.1 Re-construction, compression, and transformation . . . . . . . . . . . . . . . 10

3.4.2 Generalized differential operators across subdomains . . . . . . . . . . . . . . . 11

3.4 .3 Smoothness . . . . . . . . . . . . . . . . . . . . . . 11

3.5 Determination of model assignment . . . . . . . . . . . . . . . . . . . . . 12

3.6 Algorithmic details of PEC-implementation . . . . . . . . . . . . . . . . . . . . . . 13

3.7 Summary of the PEC-formulation . . . . . . . . . . . . . . . . . . . . . . . 14

5.1 Reference simulation . . . . . . . . . . . . . . . . . . . . . . 16

5.1 Detailed chemistry . . . . . . . . . . . . . . . . . . . . 16

5.1 .2 Skeletal chemistry . . . . . . . . . . . . . . . . . . . . 17

5.1 .3 Single-regime combustion models . . . . . . . . . . . . . . . . . . . . . 18

5.1 .4 Flame index and regime indicator . . . . . . . . . . . . . . . . . . . . 18

5.2 Pareto-efficient combustion modeling results . . . . . . . . . . . . . . . . . . . 19

5.2.1 Tradeoff between accuracy and efficiency . . . . . . . . . . . . . . . . . . 21

5.2 .2 Quantities of interest . . . . . . . . . . . . . . . . . . . . 23

5.2.3 Consideration of reaction-transport and chemistry manifolds . . . . . . . . . . . . 25

5.2 .4 Dynamic model assignment . . . . . . . . . . . . . . . . . . . . . 26

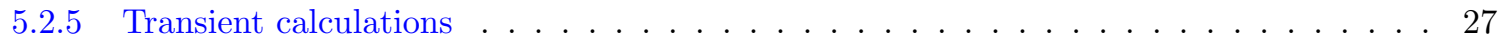

Appendix A Nomenclature

Appendix B Drift from the reaction-transport manifolds

$\begin{array}{lll}\text { Appendix } & \text { D } \quad \text { Illustration of drift operator } & \mathbf{3 6}\end{array}$

Appendix D.1 Autoignition on an inert manifold . . . . . . . . . . . . . . . . 36

Appendix D.2 Bootstrap error estimation of manifold . . . . . . . . . . . . . . . . 37

Appendix D.3 Submodel transition at interface . . . . . . . . . . . . . . . . 37

\section{Introduction}

Despite significant progress in combustion modeling, considerable challenges remain in the mathematical description and the simulation of chemically reacting flows. Reasons for this are the physico-chemical complexity, which is associated with the consideration of fluid dynamics and turbulence, the conversion of a large number of chemical species that evolve at vastly different spatio-temporal scales and concentration magnitudes, heat-release and dilatational effects due to exothermic reactions, the description of multiphase processes associated with liquid spray-phase and supercritical combustion processes, and long-range effects such as radiation and thermoacoustic interactions. While often only a subset of these processes are examined in laboratory experiments, they require consideration in practical combustion systems such as gas turbines, internal combustion engines, rocket motors, and furnaces.

Because of the computational complexity that is required to represent the oxidation of realistic fuels using detailed chemical kinetic models, lower-dimensional manifold representations are frequently used to reduce 
the dimensional complexity [1]. Common to these techniques is the representation of the thermochemical state space in terms of a reduced set of scalars who's evolution is described by the solution of transport equations. Different manifold techniques for combustion applications have been developed [1], and they can be distinguished in chemistry manifolds $[2,3,4,5]$, reaction-transport manifolds $[6,7,8,9]$, thermodynamic manifolds $[10,11,12]$, and empirical manifolds [13, 14, 15, 16].

Over recent years, several combustion models have been proposed, and many of those models rely on fundamentally different modeling approaches and approximation levels. Different categorizations have been introduced to distinguish among those models. The most common categorization is based on the combustionregime representation $[17,18,19,20,21]$, distinguishing between the asymptotic limits of premixed and nonpremixed combustion. More recently, concept-based categorizations have been introduced by differentiating between so-called flamelet-like and PDF-like methods [1]. A more general classification is to distinguish between topology-free and topology-based combustion models.

Topology-free combustion models make limited assumptions about the flame structure, and are therefore agnostic to the underlying combustion regime. These models require the solution of transport equations for all species, and employ different representations to describe scalar mixing and reaction chemistry. Examples for topology-free combustion models are finite-rate chemistry models, the eddy-dissipation concept [22], and probability-density function (PDF) methods [23, 24]. Since these models are not constrained to a particular combustion regime, they are considered to be applicable to a wider range of combustion problems. Topology-free models enable the consideration of different combustion-physical processes, such as the higher-dimensional manifold representation of the reaction chemistry, multi-stream systems, or non-adiabatic effects. However, these models are computationally expensive, require special treatment of non-local diffusion processes, and employ special discretization methods to overcome the higher-dimensional formulation in space-time-composition space. Often, topology-free models make use of chemistry manifolds to reduce the computational complexity.

Topology-based combustion models exploit the topological structure of the flame. The flame structure is then represented in terms of reaction-transport or mixing manifolds, which are obtained from the solution of representative flame configurations, such as laminar counterflow diffusion flames, freely propagating premixed flames, or one-dimensional embedded flame elements. The solution of these representative flame configurations is either evaluated prior to the simulation and stored in chemistry libraries or during the simulation to incorporate specific flow-field effects. Examples of topology-based combustion models are the class of flamelet models, including the Burke-Schumann solution [25], the steady laminar flamelet (SLF) formulation [26], the flame-prolongation in intrinsic lower-dimensional manifold (FPI) [7], the flamelet-generated manifold (FGM) method [27], and the flamelet/progress variable (FPV) formulation [9, 28].

The construction of reaction-transport manifold models introduces assumptions that are specific to the representation of the flame topology. It is therefore important to appreciate that these assumptions are directly encoded in the topology of the manifold. As such, the trust region of a particular combustion model is defined by its manifold.

Reaction-transport manifolds are parameterized in terms of a reduced set of scalars, typically consisting of mixture fraction, progress variable, enthalpy, strain or scalar dissipation rate. Because of the reduced dimensionality, these models are limited in describing certain combustion processes. To accommodate additional phenomena, these reaction-transport manifolds have been extended to consider effects of wall-heat losses [29, 30, 31], radiation [32], autoignition [33, 34, 35], multi-stream systems [36, 37, 38], and the representation of mixed- and multi-mode combustion regimes [39, 40]. While promising, these approaches face potential shortcomings: First, the validation of these extensions relies on data that might not be representative for the practical problem under consideration. Second, these model extensions may substantially increase the complexity, thereby deteriorating the accuracy in capturing the combustion behavior for which the original model was intended. Third, without prior knowledge it is not known which combustion-physical processes require consideration. Fourth, the manifold extension introduces additional unclosed contributions such as cross-dissipation terms or correlations that require modeling. Lastly, extended manifold models are applied globally although the combustion-physical processes for which they are developed are confined to a localizes region, such as the autoignition region at the flame base or the near-wall region that is affected by heat transfer and flame/surface coupling. 
Common to all applications is the issue of selecting a particular combustion model for simulating a certain flame configuration. This selection is typically guided by factors such as knowledge about the underlying combustion physics, operating conditions, quantities of interest (QoI), computational expenses, necessary model implementation efforts, and - to some extend - also by the bias of the user. Often different models provide comparable predictions for flame configurations that are represented by canonical flames, single combustion regimes, high Damköhler or low Karlovitz numbers, and simple gaseous fuels. Since, however, these combustion models invoke specific assumptions and approximations, their predictive capability reduces with increasing combustion-physical complexity.

Tasked with examining a new combustion configuration, evaluating the impact a burner-design modification has on the pollutant emissions, or assessing the potential of a new combustor concept, a practitioner faces the questions:

- Which combustion model is most adequate to accurately predict a certain quantity of interest?

- How to assess and control the accuracy of a combustion simulation in situ?

- How to balance computational cost and model accuracy during the simulation?

- How to accurately represent combustion-physical processes that are specific to a particular burner?

By addressing these questions, the objective of this work is to develop a novel Pareto-efficient combustion (PEC) framework for the dynamic utilization of different manifold representations to describe chemically reacting flows. Specifically, by combining different manifold representations, PEC enables the general adaptation of combustion submodels to the underlying flow-field representation, thereby providing an accurate description of the combustion-physical complexity. The key attributes of PEC consist in (i) the user-specific selection of a set of combustion models that can be represented by a manifold (such as chemistry, mixing, or reaction-transport manifolds), (ii) a quantity of interest such as temperature, carbon monoxide, nitric oxide, and other pollutants or intermediate species, and (iii) a cost function to describe the desirable cost and accuracy in representing the QoI. Subject to this information, a particular manifold candidate is locally selected that minimizes the cost function. PEC provides direct error control and dynamically adapts the model fidelity so that regions of different combustion-physical complexity are represented by the most appropriate model formulation; regions that are adequately represented by inert mixtures, equilibrium compositions, or quasi one-dimensional premixed or diffusion flame structures are modeled using computationally efficient reaction-transport manifold models, and topologically complex and multi-dimensional combustion processes that control flame dynamics, ignition, flame stabilization, extinction, and blow-out are described using models at higher fidelity. The flexibility in the selection of the penalty term on the cost function enables the consideration of the computational cost, since the demand for the simulation accuracy and computational expenses can vary at different stages during the model application. Beyond the specification of the set of manifolds candidates, QoI, and cost function with penalty term, PEC requires no additional user input. The local selection of the submodel is determined using the manifold drift function as metric for the model accuracy. Therefore, PEC can accommodate different combustion submodels without the requirement for expert knowledge on the model selection. Quantities of interest and desirable model accuracies are usually known requirements on the combustion simulation.

The proposed Pareto-efficient combustion modeling framework has the following main ingredients: (i) a metric for examining the proximity of the local flow-field to the combustion manifold, (ii) a procedure for assigning a combustion model locally, and (iii) a domain decomposition method to partition the flowfield domain based on the underlying combustion-physical complexity and model assignment. The first two properties are necessary to facilitate the objective assignment of combustion submodels. Since the model assignment is local, the domain decomposition capability is required to achieve optimal computational efficiency and to minimize computational overhead by treating each submodel locally.

The remainder of this paper has the following structure. The governing equations and a description of manifold models is given in the next section. The Pareto-efficient combustion modeling framework is developed in Sec. 3, and algorithmic details regarding the Pareto efficiency, the error estimation, the determination of the local model assignment, and the coupling of the different combustion submodes is provided. The subsequent sections are concerned with the application of the model to a tribrachial flame. 
The model problem is described in Sec. 4, and simulation results are presented in Sec. 5. The paper finishes with conclusions.

\section{Governing equations and manifold models}

\subsection{Governing equations}

The spatio-temporal evolution of a chemically reacting flow is described by the solution of the variabledensity Navier-Stokes equations:

$$
\begin{aligned}
D_{t} \rho & =-\rho \nabla \cdot \mathbf{u}, \\
\rho D_{t} \mathbf{u} & =-\nabla p+\nabla \cdot\left[\mu(\nabla \mathbf{u})+\mu(\nabla \mathbf{u})^{T}-\frac{2}{3} \mu \mathbf{I}(\nabla \cdot \mathbf{u})\right],
\end{aligned}
$$

where $D_{t}$ represents the substantial derivative, $\rho$ is the density, $\mathbf{u}$ is the velocity vector, $p$ is the pressure and $\mu$ is the dynamic viscosity. Conservation equations for thermochemical quantities are written in general form:

$$
D_{t} \phi=\frac{1}{\rho} \nabla \cdot\left(\rho \boldsymbol{\alpha}_{\phi} \nabla \phi\right)+\dot{\boldsymbol{\omega}}_{\phi},
$$

where $\phi \in \mathbb{R}^{N_{\phi}}$ is the thermochemical state vector, consisting of the vector of species mass fractions, $Y$, and enthalpy; $\dot{\boldsymbol{\omega}}_{\phi}$ is the corresponding source term, and the vector of scalar diffusivities is denoted by $\boldsymbol{\alpha}_{\phi}$. Equations (1) and (2) are coupled by a state equation, which is here given in implicit form:

$$
f(p, \rho, \phi)=0 .
$$

In the present study we consider the ideal gas law, so that Eq. (3) reduces to $p=\rho R T$, where $R$ is the mixture-averaged gas constant.

\subsection{Manifold methods}

Because of the large number of species that is required to describe the chemical conversion, it is usually infeasible to solve the full set of $N_{\phi}$ species transport equations in Eq. (2). As a remedy, manifold-type combustion models have been developed, in which the $N_{\phi^{-}}$-dimensional state vector is represented in terms of a low-dimensional manifold:

$$
\phi \simeq \widehat{\phi}=g(\boldsymbol{\psi}),
$$

where $\widehat{\phi} \in \mathbb{R}^{N_{\phi}}$ is the thermochemical state vector that is described by the manifold and $\psi \in \mathbb{R}^{N_{\psi}}$ is the state vector that is used to parameterize the manifold. The vector $\boldsymbol{\psi}$ may include a subset of species mass fractions, derived quantities such as mixture fraction or reaction progress variable, or other flow-field describing quantities such as strain rate or scalar dissipation. While different manifolds usually share the same definition for $\widehat{\phi}$, the structure of the transported quantities $\boldsymbol{\psi}$ and the functional relation $g$ exhibit considerable variations. For example, in the flamelet/progress variable (FPV) model, $\widehat{\phi}=g(Z, C)$ and $g$ is constructed by solving steady laminar non-premixed flamelet equations. The evolution of $\boldsymbol{\psi}$ is usually obtained as solution to the transport equation:

$$
D_{t} \boldsymbol{\psi}=\frac{1}{\rho} \nabla \cdot\left(\rho \boldsymbol{\alpha}_{\psi} \nabla \boldsymbol{\psi}\right)+\dot{\boldsymbol{\omega}}_{\psi},
$$

and the thermochemical information required to carry out the calculation can be obtained from the functional relationship in Eq. (4). Therefore, by using the manifold model, the number of equations that is solved is reduced from $N_{\phi}$ to $N_{\psi}$. Since $N_{\psi} \ll N_{\phi}$, the utilization of a manifold can significantly reduce the computational cost. In the case where $N_{\psi}=N_{\phi}$, the manifold simply reduces to that of the complete chemical mechanism.

In this study, we also require the manifold to be realizable, which ensures that $\boldsymbol{\psi}$ can be derived from the modeled state vector $\widehat{\phi}$ as

$$
\psi=h(\widehat{\phi}) .
$$




\section{Pareto-efficient combustion modeling framework}

\subsection{Preliminaries}

As discussed in Sec. 1, the PEC-framework aims to apply a spatially heterogeneous model assignment in the simulation of chemically reacting flows. To establish a mathematically well-defined formulation, we first establish the following three definitions:

- A combustion model is denoted by $m$,

- A set of candidate combustion models is denoted by $M$ so that $m \in M$,

- A model assignment mapping denoted by $m: \Omega \rightarrow M$, where $\Omega$ is the computational domain.

The introduction of the mapping $m$ enables the concise description of which submodel in $M$ is applied at a given location $\mathbf{x}$, which can be written as $m(\mathbf{x})$.

There also exists an alternative expression for the model assignment through the partition of the computational domain $\Omega$ into $N_{p}$ non-overlapping subdomains, denoted by $\Omega_{i}$. Given the model assignment mapping $M$, the partition can be uniquely determined, such that the subdomains are connected areas satisfying the following conditions:

- The union of the subdomains recovers the entire computational domain: $\cup_{i=1}^{N_{p}} \bar{\Omega}_{i}=\bar{\Omega}$;

- The subdomains are non-overlapping: $\Omega_{i} \cap \Omega_{j}=\emptyset \quad \forall i \neq j$;

- The model used in each subdomain is unique: $m(\mathbf{x})=m(\mathbf{y}) \quad \forall \mathbf{x} \in \Omega_{i}, \mathbf{y} \in \Omega_{i}$;

- the models used in adjacent subdomains are distinct: $m(\mathbf{x}) \neq m(\mathbf{y}) \quad \forall i \neq j, \mathbf{x} \in \Omega_{i}, \mathbf{y} \in \Omega_{j}, \partial \Omega_{i} \cap$ $\partial \Omega_{j} \neq \emptyset$,

where $\Omega_{i}$ denotes the interior of the $i$ th subdomain, with boundary $\partial \Omega_{i}$ and $\bar{\Omega}_{i}=\Omega_{i} \cup \partial \Omega_{i}$ denotes their union. Following the aforementioned conditions, each subdomain is equipped with a particular combustion model. For a combustion model $m$ associated with $\Omega_{i}$, we denote the corresponding thermochemical state vector as $\widehat{\phi}^{m}$ and the manifold describing solution vector as $\boldsymbol{\psi}^{m}$. Note that the dimensions of $\widehat{\phi}^{m}$ and $\boldsymbol{\psi}^{m}$ are particular to the combustion model, and can therefore differ between subdomains.

Within the interior of each subdomain, the transport equations in Eq. 5 holds as if in a single-model simulation. At the boundary $\partial \Omega_{i}$ special treatment is required. In the discrete case, this implies that the stencil of the differential operator contains elements of different subdomains. Details of the coupling will be discussed in Sec. 3.4. In the most general setting, other physical submodels and discretization techniques can be utilized across subdomains [41, 42].

The rest of Sec. 3 focuses on obtaining the model assignment mapping $m$. The concept of the domain partition is an intuitive description of the model utilization and is especially convenient for discussing the coupling between different models. However the partition is derived from the model assignment mapping $m$, rather than being sought explicitly.

\subsection{Pareto efficiency of combustion models}

Simulation accuracy and computational cost are main criteria for reacting flow simulations. When multiple computational models are available for a particular application, the decision which model to use is usually made by considering the trade-off between these two criteria. To rationalize the procedure, we can formally characterize a simulation that is obtained with a particular model by a modeling error $\mathcal{E}$ and a computational cost $C$. With this, each simulation can be mapped to the $\{\mathcal{E}, C\}$-performance space. An example for single-model simulations is schematically illustrated in Fig. 1, showing the performance of different combustion models as a function of normalized model error and normalized computational cost. The selection of an optimal model can then be made from the Pareto set in the $\{\mathcal{E}, C\}$-performance space. This Pareto set is represented by all models that offer the lowest error for a defined level of cost or - in turn - the lowest cost for a given level of error; any other model from this set is suboptimal; models beyond this Pareto frontier are unachievable [43]. The extension of the Pareto frontier to access these conditions requires further model development that can be made at any level of computational complexity, as was addressed in Sec. 1 . 
Since the Pareto frontier is specific for each combustion problem and most-likely dependent on additional model-specific parameters, such as mesh-resolution, time-to-convergence, subgrid models, or chemical mechanism, the direct evaluation of the Pareto front becomes infeasible for any practical application. However, by introducing this concept, a main component of PEC is to provide a mathematical rigorous formalism for the optimal model selection.

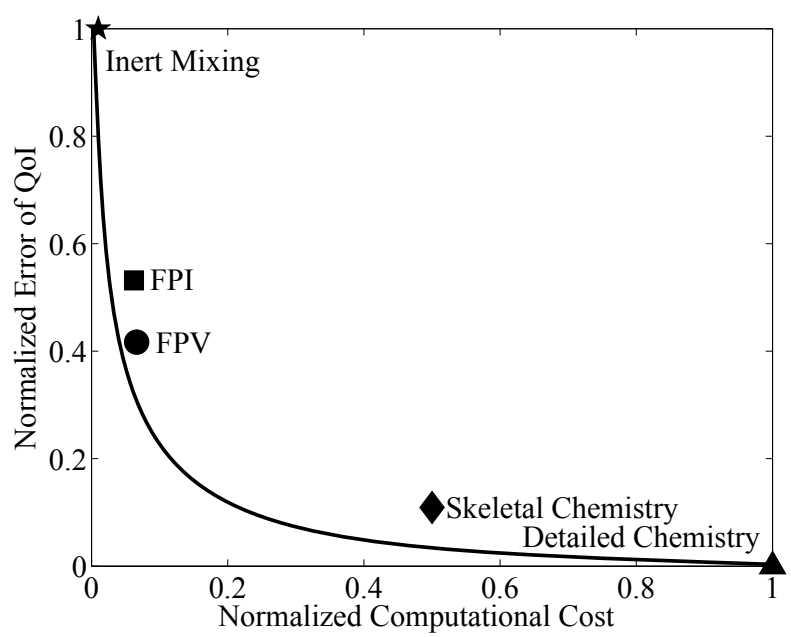

Figure 1: Schematic illustration of the Pareto front, representing the computational cost and model error in predicting a certain quantity of interest.

For a simulation that employs a single combustion model, the Pareto frontier can be obtained as the solution of the following optimization problem:

$$
\begin{aligned}
& \min _{m: \Omega \rightarrow M} \mathcal{E}(m)+\lambda C(m), \\
& \text { subject to } m(\mathbf{x})=m(\mathbf{y}) \quad \forall \mathbf{x}, \mathbf{y} \in \Omega,
\end{aligned}
$$

where $\lambda$ is a Lagrange multiplier to enable traversing the Pareto frontier. The optimal input argument we are seeking is the model assignment mapping $m(\mathbf{x}): \Omega \rightarrow M$ introduced in Sec. 3.1. The single-model requirement constrains the flexibility of $m$ so that its output has to be a single value over the entire domain.

Although Eq. 7 offers an objective approach for selecting a particular combustion model, the so obtained model assignment $m$ applies a single model over the entire computational domain. As such, the solution to Eq. 7 can provide only a suboptimal solution for capturing combustion-physical processes that are confined to local flow regions. In addition, a uniform model assignment only provides a discrete Pareto frontier due to the limited possible outcomes of the optimization procedure. Therefore, a smooth transition from low-fidelity to high-fidelity simulations cannot be achieved.

Both of the aforementioned issues can be resolved by allowing a more flexible and spatially heterogeneous model assignment. In the optimization framework, this requires eliminating the constraints on $m$ and formulating $\mathscr{E}$ and $C$ such that they are sensitive to variations in the local model assignment. Hence the optimization problem becomes

$$
\min _{m: \Omega \rightarrow M} \mathcal{E}(m)+\lambda e(m),
$$

where $\mathcal{E}$ and $\mathcal{C}$ are defined as the $L^{p}$-norm of local quantities $e$ and $c$,

$$
\begin{aligned}
& \mathscr{E}(m)=\int_{\Omega}\left|e^{m}(\mathbf{x})\right|^{p} d \mathbf{x}, \\
& \mathcal{C}(m)=\int_{\Omega}\left|c^{m}(\mathbf{x})\right|^{p} d \mathbf{x} .
\end{aligned}
$$


In the following we will consider the $L^{1}$-norm to simplify the notation. It is worth mentioning that the formulation in Eq. 8 is an instantaneous cost function, and the optimization can be considered as a greedy approach to a transient problem.

So far, we did not provide a mathematical prescription for $e$ and $c$, which will be given in the following section. The coupling strategy is introduced in Sec. 3.4 to accommodate the spatially heterogeneous combustion model assignment, and the treatment at the interface of the subdomains. The description of the PEC-framework is then completed by discussing the optimization strategy and the solution methods for finding $m$ in Eq. 8, which will be presented in Sec. 3.5.

\subsection{Computational cost and error estimation}

The computational cost of a particular combustion model is determined by the number of independent scalars that are solved for, the source-term evaluation, data retrieval from the library interpolation, and the solution of auxiliary expressions to evaluate closure models and constitutive relations. As such, the local computational cost $c^{m}(\mathbf{x})$ in Eq. $9 \mathrm{~b}$ depends solely on the local model used. Therefore it can only take value from a set of constants i.e.

$$
c^{m}(\mathbf{x})=c_{m},
$$

where $m=m(\mathbf{x})$. The constant $c_{m}$ is a property of each combustion model. Its value can be predetermined prior to the simulation, and provided as a user input to the PEC-formulation. In practice, the computational cost of each model depends on the numerical method, parallelization strategies, hardware architecture, and other factors. Since these conditions may vary on a case-by-case basis, but otherwise introduce no conceptual difficulties in the PEC-formulation, it is not the focus of this study to discuss how to accurately determine values for these constants.

In contrast, the calculation of the model error $\mathcal{E}(m)$ requires information about the flow field. Since $e^{m}(\mathbf{x})$ is a local measure of the model error with respect to the model assignment $m$, a canonical choice for determining $e^{m}(\mathbf{x})$ is to relate the model error to the difference between the true state and the modeled state: $\Delta=\phi-\widehat{\phi}$. Hence, the evaluation of this expression requires knowledge about the true state $\phi$, and a complementary detailed simulation is necessary. Since this introduces substantial overhead, the direct approach becomes infeasible for any practical application. To overcome this issue, it is proposed to quantify the model error by considering the dynamics on the model's manifold. This is further discussed in the next section.

\subsubsection{Drift from manifold}

Consider the interior of the subdomain $\Omega_{i} \subseteq \Omega$, to which a combustion model $m$ is locally applied. Discretely, it means that the stencil of all differential operators are within $\Omega_{i}$. The modeled quantity $\widehat{\phi}^{m}(\mathbf{x})$ is directly evaluated using the functional relationship described in Eq. 4. Therefore, its dynamics is implicitly governed by the following expression:

$$
D_{t} \widehat{\boldsymbol{\phi}}^{m}(\mathbf{x})=\frac{\partial \widehat{\boldsymbol{\phi}}^{m}}{\partial \boldsymbol{\psi}^{m}}(\mathbf{x}) \cdot D_{t} \boldsymbol{\psi}^{m}(\mathbf{x}),
$$

and the evolution of the true thermochemical state $\phi$ is determined by Eq. 2. The difference between both solutions embeds the inconsistency between the dynamics of $\phi$ and $\widehat{\phi}$, representing the error for using the model $m$. In this section, we will develop a new measure of proximity by assessing this inconsistency. This analysis employs formalism developed by Pope [1] to examine multidimensional combustion manifolds in composition space.

To establish a quantitative assessment of the model inconsistency, we consider the drift of $\phi$ with respect 
to $\widehat{\phi}$. Mathematically, this can be written as:

$$
\begin{aligned}
\mathscr{D}^{m} & =\left.D_{t} \boldsymbol{\Delta}^{m}\right|_{\boldsymbol{\Delta}=\mathbf{0}}, \\
& =\left.D_{t} \boldsymbol{\phi}\right|_{\boldsymbol{\phi}=\widehat{\boldsymbol{\phi}}^{m}}-\frac{\partial \widehat{\boldsymbol{\phi}}^{m}}{\partial \boldsymbol{\psi}^{m}} \cdot D_{t} \boldsymbol{\psi}^{m}, \\
& =\left[\frac{1}{\rho} \nabla \cdot\left(\rho \boldsymbol{\alpha}_{\phi} \nabla \boldsymbol{\phi}\right)+\dot{\boldsymbol{\omega}}_{\phi}\right]_{\boldsymbol{\phi}=\widehat{\boldsymbol{\phi}}^{m}}-\frac{\partial \widehat{\boldsymbol{\phi}}^{m}}{\partial \boldsymbol{\psi}^{m}} \cdot\left[\frac{1}{\rho} \nabla \cdot\left(\rho \boldsymbol{\alpha}_{\psi^{m}} \nabla \boldsymbol{\psi}^{m}\right)+\dot{\boldsymbol{\omega}}_{\psi^{m}}\right],
\end{aligned}
$$

where $m=m(\mathbf{x})$. This equation describes the initial growth rate of the manifold error. It is therefore referred to as the drift from manifold, and denoted by $\mathscr{D}$. This drift term is defined with respect to the model assignment $m$, and includes the spatial relationship among submodels.

Note that the error estimation of a lower-order model through Eq. 12 only requires information about thermochemical states on the manifold $(\widehat{\phi})$ and the topology of the manifold. This is a bootstrapping method, since it does not require knowledge of the actual thermochemical state $\phi$. In addition, this procedure does not need information on how the manifold is constructed or what model assumptions are introduced. These properties alleviate the demand for the examination of model assumptions and their consequences, which would require expert knowledge from the user and, as mentioned in Sec. 1, is often impractical in complex configurations. This is also particularly attractive for application to empirical manifolds, since no assumptions are explicitly specified for data-driven models.

Point-local terms in the expression for $\mathscr{D}$ are the transport properties $\left(\boldsymbol{\alpha}_{\psi},\left.\boldsymbol{\alpha}_{\phi}\right|_{\phi=\widehat{\phi}}\right)$, the chemical source terms for both $\boldsymbol{\phi}$ and $\boldsymbol{\psi}\left(\dot{\boldsymbol{\omega}}_{\psi},\left.\dot{\boldsymbol{\omega}}_{\phi}\right|_{\phi=\widehat{\phi}}\right)$, and the manifold Jacobian $(\partial \widehat{\boldsymbol{\phi}} / \partial \boldsymbol{\psi})$. For pre-tabulated manifold models, these terms can be directly retrieved from the table since the thermochemical state resides on the manifold as defined in Eq. 12, i.e. $\phi=\widehat{\phi}^{m}$. For manifold models that do not use a tabulation method, these quantities can usually be computed at a reduced cost. The cost for computing $\mathscr{D}^{m}$ is further reduced by the fact that the evaluations are only performed with respect to QoI, the number of which is considerably smaller than the total number of species.

In contrast, the diffusion terms are non-local. For the case that submodels differ between neighboring locations (corresponding to the interface between model assignments), these terms would require special treatment. The treatment needs to extend the differential operators to the interface, thereby utilizing the non-local terms as a continuity sensor and preventing the local usage of certain models that produce discontinuous scalar fields. This point will be further discussed in Sec. 3.4 when the coupling strategy is introduced.

In the interior of a subdomain, Eq. 12 can be related to the curvature of the manifold and the local scalar dissipation rates of $\boldsymbol{\psi}$ as derived by Pope [1]. The drift, $\mathscr{D}$, becomes physically insightful in terms of interpreting the source of errors for a given combustion manifold. Applying similar analysis to a set of reaction-transport manifolds and chemistry manifolds, the expressions of $\mathscr{D}$ are obtained and summarized in Appendices Appendix B and Appendix C. In this analysis, it is shown that for reaction-transport manifolds, deviations are initiated by the discrepancy between the scalar dissipation rate of the flow field $\chi$ and the modeled scalar dissipation rate $\widehat{\chi}$ that is weighted by the manifold curvature, which is largest in regions of chemical reactivity. This analysis also shows that by extending the manifold, the increased dimensionality of $\boldsymbol{\psi}$ would unavoidably introduce new sources of error.

\subsubsection{Cost function for model error}

In the previous section, we have established the concept of the manifold drift, which can be used to assess the inconsistency between the dynamics of the approximated and the true states. However, we have not established its relation to $e^{m}(\mathbf{x})$. To derive a quantitative measure for assessing the model accuracy, we first introduce the notation of a quantity of interest $Q$, containing a set of scalar quantities that are of interest to the user. With this, we can determine the accuracy of a particular model by measuring the norm of $\mathscr{D}^{m}$ projected onto the subspace that is spanned by $Q$ :

$$
e^{m}=\frac{1}{|Q|} \sum_{\alpha \in Q}\left|\frac{\mathscr{D}_{\alpha}^{m}}{C_{\alpha}}\right|^{p},
$$


where $|Q|$ denotes the cardinality of $Q$ and $B_{\alpha}$ is a scaling factor, which is chosen to be the reference chemical source term in this study. We consider the $L^{1}$-norm to simplify the notation in this study. Since only quantities that are contained in $Q$ are used in evaluating $\mathscr{D}$, the added overhead in evaluating Eq. 13 is minimal.

The new measure for the proximity between $\widehat{\phi}$ and $\phi$ that is defined by Eq. 13 is used as the cost function for the local error. This quantity is sensitive to the local scalar and velocity fields, and has a general form that can be applied to all manifold models.

\subsection{Coupling strategy at the interface of subdomains}

PEC utilizes a spatially heterogeneous model assignment. Due to the existence of non-local differential operators in the transport equations, a strategy is required to consistently couple the different submodels. This coupling strategy should enable a physical and consistent calculation of the system dynamics under the spatially heterogeneous mapping $m$ as well as the extension of the drift term $\mathscr{D}$ in Eq. 12 from the interior to the interface of subdomains.

\subsubsection{Re-construction, compression, and transformation}

At each time step, two different solution fields can be directly obtained: the model assignment field $m(\mathbf{x})$ and the corresponding manifold describing solution vector field written as $\boldsymbol{\psi} \mid m$. Note that $\boldsymbol{\psi} \mid m(\mathbf{x})=$ $\boldsymbol{\psi}^{m}(\mathbf{x})$, where $m=m(\mathbf{x})$. To conform between the current model assignment $m$ and its alternative $m^{\prime}$ the transformation operator $T$ is introduced. This operator consists of two procedures: the re-construction operator $G$ and the compression operator $H$.

The global re-construction operator $G^{m}$ is used to obtain the thermochemical state vector with respect to the mapping $m$. This procedure combines the local operator $g^{m}$ defined in Eq. 4 and can be written as

$$
\begin{aligned}
\widehat{\phi} \mid m(\mathbf{x}) & =G^{m}(\boldsymbol{\psi} \mid m(\mathbf{x})) \\
& =g^{m}\left(\boldsymbol{\psi}^{m}(\mathbf{x})\right) \\
& =\widehat{\boldsymbol{\phi}}^{m}(\mathbf{x}),
\end{aligned}
$$

where $m=m(\mathbf{x})$. The compression operator $H^{m^{\prime}}$ then converts $\widehat{\phi}$ obtained from Eq. 14 to the field of $\boldsymbol{\psi}$ with respect to the new model assignment $m^{\prime}$. This procedure unitizes the local operator $h^{m^{\prime}}$ defined in Eq. 6 and can be written as

$$
\begin{aligned}
\boldsymbol{\psi} \mid m^{\prime}(\mathbf{x}) & =\boldsymbol{\psi}^{m^{\prime}}(\mathbf{x}) \\
& =h^{m^{\prime}}\left(\widehat{\boldsymbol{\phi}}^{m}(\mathbf{x})\right) \\
& =H^{m^{\prime}}(\widehat{\boldsymbol{\phi}} \mid m(\mathbf{x})),
\end{aligned}
$$

where $m=m(\mathbf{x})$ and $m^{\prime}=m^{\prime}(\mathbf{x})$. By composing the re-construction and compression operators, we introduce the transformation operator,

$$
T^{m^{\prime}, m}=H^{m^{\prime}} \circ G^{m},
$$

which combines Eqs. 14 and 15 to

$$
\boldsymbol{\psi} \mid m^{\prime}(\mathbf{x})=T^{m^{\prime}, m}[\boldsymbol{\psi} \mid m(\mathbf{x})]
$$

A similar form holds for the local transformation operator $t^{m^{\prime}, m}=h^{m^{\prime}} \circ g^{m}$. The so obtained $\boldsymbol{\psi} \mid m^{\prime}(\mathbf{x})$ is in compliance with the definition of both $\widehat{\phi} \mid m$ and $\boldsymbol{\psi} \mid m^{\prime}$. It is worth noting that $t^{m, m^{\prime}}$ and $t^{m^{\prime}, m}$ are in general not inverse operators of each other and $t^{m^{\prime}, m}$ becomes an identity operator, if $m$ and $m^{\prime}$ share the same definition of the solution vector $\psi$. 


\subsubsection{Generalized differential operators across subdomains}

For a spatially heterogeneous model assignment mapping $m$, the transport equation defined in Eq. 5 and the drift from the manifold, defined in Eq. 12, are well-defined in the interior of each subdomain, since the differential operators are applied within a single subdomain. However, at the interface of each subdomain, $\psi$ changes its definition across the interface and thus the differential operators become ill-defined. Since the issue lies within the differential operators, if certain treatment makes them applicable at the interface of subdomains, both the transport equation in Eq. 5 and the error estimation in Eqs. 12 and 13 can be extended to the entire domain including the interface.

The treatment is achieved with the notion of the transformation operator $t^{m, m^{\prime}}=h^{m} \circ g^{m^{\prime}}$. At the interface, for all points $\mathbf{y}$ included in the stencil of $\mathbf{x}$ such that $m^{\prime}=m^{\prime}(\mathbf{y}) \neq m=m(\mathbf{x})$, we can temporarily conform $\boldsymbol{\psi}^{m^{\prime}}(\mathbf{y})$ to $\boldsymbol{\psi}^{m}(\mathbf{y})$ as

$$
\boldsymbol{\psi}^{m}(\mathbf{y})=t^{m, m^{\prime}}\left(\boldsymbol{\psi}^{m^{\prime}}(\mathbf{y})\right) .
$$

This procedure can be expressed in shorthand as $t^{m(\mathbf{x})}$. The so obtained $\boldsymbol{\psi}^{m}$ is temporarily defined within the stencil and shares a unified definition. Hence, differential operators can be applied. In other words, the differential operators for $\boldsymbol{\psi}$ are generalized by the composition with the transformation operator $t^{m(\mathbf{x})}$. At the interior of the subdomain, $t^{m(\mathbf{x})}$ reduces to the identity operator and the original differential operators are recovered.

With the generalized differential operators, the solution vector $\boldsymbol{\psi}$ over the entire domain can be tightly coupled in a consistent fashion. In the case where $N_{\psi}=N_{\phi}$, the entire thermochemical state vector can be coupled by this strategy. However, for most combustion models, $N_{\psi} \ll N_{\phi}$ and thus there are many components in $\psi$ that are not treated by this strategy. Such scenario will be discussed next.

\subsubsection{Smoothness of $\widehat{\phi}$ and $\boldsymbol{\psi}$}

The smoothness of the thermochemical state vector $\widehat{\phi}$ and manifold solution vector $\psi$ is important for two reasons. First, $\widehat{\phi}$ is the model approximation of the true thermochemical state $\phi$. If the physical solution is smooth, a good model approximation should preserve this property. Second, a discontinuous $\widehat{\phi}$-field may lead to a discontinuous density field, thereby introducing numerical instabilities.

The smoothness of $\widehat{\phi}$ and $\boldsymbol{\psi}$ is defined by their differentiability. In the case of $\boldsymbol{\psi}$, its differentiability is extended by the generalization introduced in Sec. 3.4.2. Since the compression operator $h$ is always differentiable for a well-defined combustion model, the smoothness of $\widehat{\phi}$ guarantees that of $\boldsymbol{\psi}$, but not vice versa due the change of the functional relationship between $\widehat{\phi}$ and $\boldsymbol{\psi}$ across the interface. One special case is the interface between FPV and FPI models that have the same definition of mixture fraction $Z$ and progress variable $C$. The field of $\psi,\{Z, C\}$ in this case, is shared by both models and therefore it is always differentiable. However, $\widehat{\phi}$ may be discontinuous at the interface. As a result, it is in general much easier to preserve the smoothness for $\boldsymbol{\psi}$ than for $\widehat{\phi}$. In fact, if the model assignment $m$ is kept unchanged in time, the diffusion operator in Eq. 5 would naturally guarantee the smoothness of $\boldsymbol{\psi}$ over the entire domain including the interfaces.

The question then becomes how to find a model assignment that ensures the smoothness of $\widehat{\phi}$ and does not disturb the smoothness of $\boldsymbol{\psi}$. The solution lies in the error estimator $\mathscr{D}$ introduced in Eq. 12. Since non-smooth fields of $\widehat{\phi}$ and $\psi$ imply inaccurate predictions, a desirable error estimator should recognize this. In fact, the smoothness of $\widehat{\phi}$ and $\boldsymbol{\psi}$ is examined by the diffusion operators in $\mathscr{D}$. As a result, a discontinuous $\widehat{\phi}$ and $\psi$ will lead to an unbounded cost function for the model error or a very large value in the discrete case. Therefore, this model assignment is reflected by a large value of $\mathscr{D}$ and therefore discouraged. In regard to the discontinuity for the components of $\widehat{\phi}$ that are not included in $Q, e^{m}$ will not be influenced. Consequently, these situations are not penalized by the current formulation. 


\subsection{Determination of model assignment}

The optimal model assignment is determined by the solution to the optimization problem in Eq. 8. In the discrete form, assuming a uniform grid without loss of generality, this can be written as:

$$
\min _{m: \Omega \rightarrow M} \sum_{i=1}^{|\Omega|}\left|e^{m}\left(\mathbf{x}_{i}\right)\right|^{p}+\lambda\left|c^{m}\left(\mathbf{x}_{i}\right)\right|^{p} .
$$

An exhaustive search to this problem requires the evaluation of the cost function with respect to all possible model assignments. Given the current assignment $m$, one can utilize the transformation and re-construction operators to obtain $\widehat{\phi} \mid m^{\prime}$ and $\boldsymbol{\psi} \mid m^{\prime}$,

$$
\begin{aligned}
& \boldsymbol{\psi} \mid m^{\prime}=T^{m^{\prime}, m}(\boldsymbol{\psi} \mid m), \\
& \widehat{\phi} \mid m^{\prime}=G^{m^{\prime}}\left(\boldsymbol{\psi} \mid m^{\prime}\right),
\end{aligned}
$$

and subsequently evaluate $\mathscr{D}^{m^{\prime}}$ and $e^{m^{\prime}}$. Such exhaustive search requires $\mathcal{O}\left(|M|^{|\Omega|}\right)$ operations, $|M|$ being the number of candidate models and $|\Omega|$ being the number of grid points, which is computationally intractable.

To overcome this issue, it is possible to reduce the problem to the following point-local minimization problem:

$$
\min _{m\left(\mathbf{x}_{i}\right) \in M}\left|e^{m}\left(\mathbf{x}_{i}\right)\right|^{p}+\lambda\left|c^{m}\left(\mathbf{x}_{i}\right)\right|^{p},
$$

under the condition that both $e^{m}\left(\mathbf{x}_{i}\right)$ and $c^{m}\left(\mathbf{x}_{i}\right)$ are localized variables, i.e., they only depend on the model assignment at $\mathbf{x}_{i}$ and are not affected by the model selection at neighboring points. This point-local minimization requires only $\mathcal{G}(|m| \cdot|\Omega|)$ operations for an exhaustive search. Intuitively, this condition is not satisfied since the adjacency between two locally incompatible models may result in large modeling errors. This intuition is mathematically confirmed for the current choice of $e^{m}$ due to the diffusion terms in Eq. 12, which penalizes the aforementioned scenario. The irreducible problem is very similar to minimizing Potts energy, which is a known NP-hard problem [44]. As a result, certain approximations have to be introduced in the optimization procedure to make this problem tractable.

For the present application, we adopt the point-local cost function which enables the use of Eq. 22. The optimal local model assignment is determined by assuming that the neighboring models are kept the same as in the last time step. Note that this approximation should not be confused with the approximation in the cost function, which is still computed exactly. The resulting procedure is substantially more efficient and fully compatible with a domain decomposition for parallel simulations. It is worth mentioning that with the current framework it is also possible to use more sophisticated approximation algorithms, e.g. those mentioned in [44]. There are two reasons behind the current choice. The first is the computational cost and simplicity for parallelization and implementation. The second is that $e^{m}\left(\mathbf{x}_{i}\right)$ is an inference of the error, based on the current solution, which itself is improving over time. A too aggressive step-wise optimization procedure, especially early in the simulation, may not result in a better outcome over the dynamic procedure.

With the approximation in Eq. 22, we now discuss a special case to illustrate the behavior of this procedure. This example also provides guidance for selecting $\lambda$. Consider a candidate model set with two elements. Model 1 is a detailed chemistry model and model 2 is a low-order model. Note that by definition, $e^{m}\left(\mathbf{x}_{i}\right)=0$, if $m\left(\mathbf{x}_{i}\right)=1$. We also normalize the computational cost so that the cost of the detailed full chemistry model is one, i.e. $c_{1}=1$. Equation 22 thus gives a simple thresholding algorithm:

$$
m\left(\mathbf{x}_{i}\right)= \begin{cases}2 & \text { if } e^{m}\left(\mathbf{x}_{i}\right) \mid\left(m\left(\mathbf{x}_{i}\right)=2\right)<\epsilon \\ 1 & \text { otherwise }\end{cases}
$$

where the threshold $\epsilon=\lambda\left(1-c_{2}\right)$. Therefore, the penalty factor $\lambda$ can be interpreted as the tolerance for the estimated error of a model with negligible cost. The extension to a larger set of models directly follows from this analysis. 


\subsection{Algorithmic details of PEC-implementation}

In this section, we outline essential components of the PEC-framework. These consist in a set of combustion models $M$, a set of quantities of interest $Q$, and the specification of the penalty factor $\lambda$. Each model $m \in M$ is associated with the corresponding $\operatorname{cost} c_{m}$ and a scaling factor $B$ for each quantity of interest in $Q$. Algorithm 1 provides a description with the implementation details of the PEC-framework.

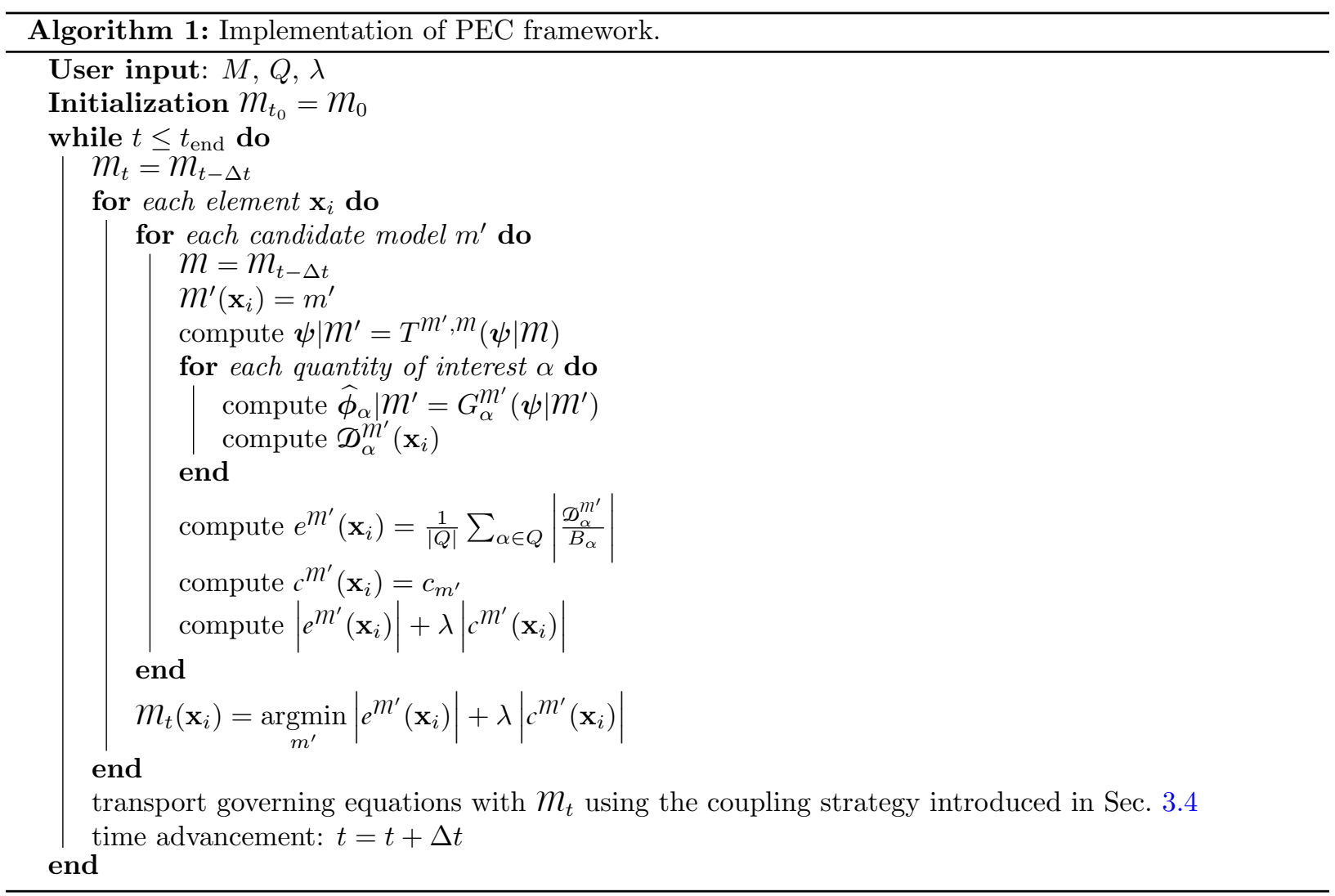

At each time step, the PEC-algorithm iterates over each grid point. For each candidate model, it computes the corresponding $\mathscr{D}$ for all quantities of interest. With this, the local model error $e^{m}$ is evaluated by contracting and rescaling $\mathscr{D}$. The cost function is then obtained by including the pre-defined computational cost with the weight $\lambda$. With this information, this submodel is then locally selected that has the lowest cost function. After each grid point is assigned to a model, subdomain boundaries are identified to apply coupling strategies and solve the transport equations for the next time step. To accommodate heterogeneous computational cost due to the different submodel-assignments, this step can be combined with a domain-decomposition step.

The cost associated with the model assignment procedure scales with $\mathcal{\Theta}(|M| \cdot|Q| \cdot|\Omega|)$. Global operators $G$ and $T$ are used in Algorithm 1 for clarity. The actual operation requires only local changes since $m^{\prime}$ and $m$ are different only at one point. As discussed in Sec. 3.3, the overhead for this procedure is rather modest for a reasonable number of candidate models and quantities of interest.

We close the description of the PEC-framework by remarking on the model regularization. As many approximate optimization problems, additional regularization may help to guide the behavior of the outcome and to ensure certain properties for practical considerations. Examples are the prevention of a frequent switching of the local model assignment, the suppression of hysteresis effects, the consideration of a sufficiently large subdomain, or the contraction of the number of subregions. All of these requirements can be accommodated in the herein proposed PEC-framework. 
To avoid frequent model reassignment, a switching penalty can be added to Eq. 22, which takes a positive constant when the current local assignment is different from the last one and is zero if the local assignment is unchanged. A "momentum" factor can be used to treat hysteresis effects. Instead of updating $e_{t+\Delta t}^{m}$ as is, one can blend the current value with the previous results: $e_{t+\Delta t}^{m}=(1-\delta) e_{*}^{m}+\delta e_{t}^{m}$, where $e_{*}^{m}$ is the latest evaluated error estimation. This is a common treatment in stochastic optimization [45], effectively applying a one-sided exponential filter (with filter width $-1 / \log (\delta)$ ) to the cost function so that the hysteresis effect is accounted for. To eliminate unreasonably small subdomain assignments, one can introduce a Potts energy term [44] to the cost function or apply explicit spatial filtering to the model assignment via binary pseudo-coding $[46,47]$.

\subsection{Summary of the PEC-formulation}

Before applying the PEC-formulation to a flame configuration, we conclude this section by summarizing the framework and key concepts. PEC evolves around an inherently dynamic procedure of a combustionmodel assignment, $m$. As shown in Algorithm 1, the model assignment is made based on the current state of the CFD-solution. The updated model assignment is then fed back to the CFD-calculation and used to advance the thermochemical state. Since both $m$ and the CFD-solution evolve over time, the PEC-framework can be readily applied to transient calculations, and this is further discussed in Sec. 5.

Based on the current solution of the reactive scalars, $m$ is updated through an approximate solution to Eq. 8. The optimization problem, defined in Eq. 8, formalizes the trade-off between accuracy and efficiency. Efficiency is measured by the local estimation of the computational cost. As shown in Eq. 10, the cost estimation takes values from a set of pre-defined constants associated with the candidate models, and a more detailed evaluation of the cost of each submodel can be provided to the PEC-framework, taking into account operating conditions, numerical evaluation procedures, and other factors. The accuracy is assessed by the local error estimation defined in Eqs. 12 and 13. The local error estimation is a function of the selected model at this particular location, models applied at the surrounding area, and the current solution of the quantities of interest.

The drift, defined in Eq. 12, measures how fast the thermochemical state would depart from the current manifold if the complete set of transport equations were solved. Therefore, it provides a local error estimation that does not require information about the true state. The transport properties and reaction rates, required in Eq. 12, correspond to the thermochemical state residing on the manifold, $\widehat{\phi}$. Thus, these terms can either be pre-tabulated or calculated at a reduced cost. Furthermore, at the interface between different models, the diffusion operators in Eq. 12 examine the compatibility between models and prevent inappropriate adjacencies of models. Since only the components of $\mathscr{D}$ corresponding to the quantities of interest are needed in Eq. 13, the cost for the error estimation is further reduced.

The coupling between subdomains is realized by a generalization of the differentiation operators. This generalization utilizes compression and reconstruction operators to communicate between neighboring submodels. Smoothness of the scalar fields for the quantities of interest is guaranteed through the specification of the error estimation, which penalizes the adjacency between incompatible submodels.

\section{Model problem: Tribrachial flame}

To demonstrate the PEC-framework, we consider a tribrachial flame configuration as a test case. This flame configuration contains different combustion regimes to challenge the accuracy of most single-regime combustion models. A tribrachial flame, shown schematically in Fig. 2, consists of lean and rich premixed flame branches, and a diffusion flame is embedded between both branches. Within the diffusion flame, excess reactants from the rich and lean premixed flame branches oxidize [48]. The three flame branches meet at the triple point of nearly stoichiometric composition. Furthermore, the mixture in the diffusion branch is close to chemical equilibrium and the diffusion flame is less prominent than the two premixed flames [49]. Apart from the different flame regimes, tribrachial flames exhibit complex combustion-physical processes that are complicated by curvature, stretch rate, and preferential diffusion processes, therefore affecting the 


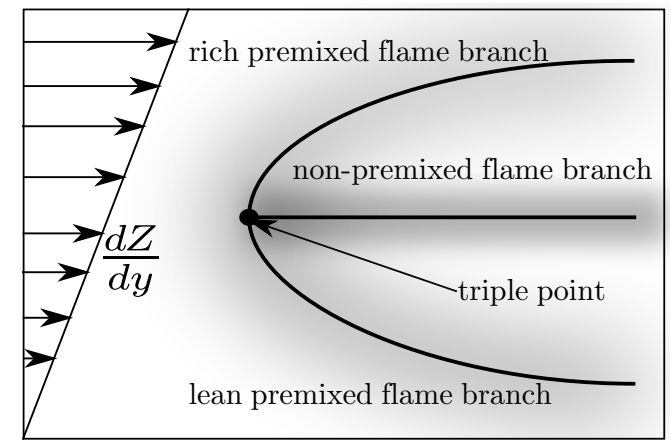

Figure 2: Schematic of the flame structure for a tribrachial flame.

mass-burning rates in the premixed flame branches, radical production, and variations of the propagation speed $[50,48,51,52]$.

In the past, laminar tribrachial flames have been considered for the development and assessment of combustion models by different groups. Oijen and de Goey [49] studied the relationship between flame propagation and the level of stratification using the FGM model. In their study, a two-dimensional manifold with progress variable and mixture fraction as the controlling variables were used. The structure and behavior of flamelets in both premixed and diffusion flame branches were reported. Knudsen and Pitsch [39] proposed a mixed-regime flamelet model by linearly combining asymptotic premixed and diffusion flames. A new regime indicator was developed to allow the application of premixed and non-premixed flamelet models.

The tribrachial flame configuration under consideration is a 2D laminar methane-air flame. The level of stratification is quantified by the mixture fraction gradient in the transverse direction $d Z / d y$. Using the specified value of $d Z / d y$, a linear profile of mixture fraction with the corresponding slope is prescribed at the inflow, and the stoichiometric value of the profile is centered in the computational domain. The profile is also bounded by the realizable values of the mixture fraction. At the inlet, the mixture is assumed to be unreacted so that the species composition is fully described by the mixture fraction alone. The inlet velocity profile is prescribed by a plug flow.

Throughout this work, we specify $d Z / d y=L^{-1}$ with $L=2 \mathrm{~cm}, T=300 \mathrm{~K}$ and $p=1$ bar for all simulations. This level of stratification leads to a tribrachial flame envelope that occupies a significant area of the domain. At the same time, the flame envelope has sufficient clearance from the lateral no-slip wall boundaries to avoid flame-wall interaction. The chemistry is described by the GRI-3.0 mechanism [53], and the steady flamelet calculations are performed using the FlameMaster code [54].

The numerical solution is obtained using a variable-density low-Mach number solver [9]. The temporal discretization for this solver is a two stage predictor-corrector scheme with a pressure Poisson corrector step. Detailed chemistry is solved using a Strang splitting scheme. Although the tribrachial flame is laminar, the flame is required to be stabilized at the same location for model comparison. Therefore, a proportionalintegral-derivative (PID) controller [55] is utilized to adjust the inlet velocity.

The computational domain is $0.75 \mathrm{~L} \times L$ in the transverse and streamwise directions respectively. The mesh for all computations uses $800 \times 600$ grid points with uniform mesh size. The corresponding element length is $20 \mu \mathrm{m}$, corresponding to approximately 20 grid points across a planar stoichiometric methane-air premixed flame at the same operating conditions. This is sufficient for this simulation and is verified by grid convergence studies. All simulations are performed using unity Lewis number approximation.

In the following, simulations with different combustion models are performed. These include reactiontransport manifold and skeletal chemistry-manifold models. These models are summarized in Table 1. The inert mixing model (IM) is constructed from the mixing solution. The chemical source terms are precomputed using detailed kinetics. The non-reacting system (including the chemical source terms) is fully described by $\boldsymbol{\psi}=Z$. The FPV and FPI models are the two other reaction-transport manifolds, in which the solutions are obtained from premixed and non-premixed steady flamelets, respectively. In addition to the detailed chemistry solution with the GRI-3.0 mechanism, we consider a second skeletal chemistry-manifold 
model, in which the chemistry is described by a 19-species reduced mechanism [56].

The computational cost of each model is estimated by the corresponding number of transported quantities, normalized by the number of species in the detailed chemistry model. This is a rather conservative estimation, which neglects overhead that is required for evaluating the Jacobian and the often severe timestep restriction of chemistry-manifold models due to the chemical stiffness.

\begin{tabular}{|c||c|c|c|l|}
\hline Model & Abbreviation & $\boldsymbol{\psi}^{m}$ & Cost, $c_{m}$ & Comment \\
\hline \hline Detailed Chemistry & DC & $\phi$ & 1 & GRI-3.0 [53], 53 species \\
\hline Skeletal Chemistry & SC & $\phi$ & 0.5 & DRM-19 [56], 19 species \\
\hline Flamelet/Progress Variable & FPV & $(Z, C)^{T}$ & 0.02 & $\begin{array}{l}\text { Non-premixed flamelet solu- } \\
\text { tion, unity Lewis number, } \\
\text { GRI-3.0 }\end{array}$ \\
\hline Flamelet-Prolongation of ILDM & FPI & $(Z, C)^{T}$ & 0.02 & $\begin{array}{l}\text { Premixed flamelet solution, } \\
\text { unity Lewis number, GRI- } \\
3.0\end{array}$ \\
\hline Inert Mixing & IM & $Z$ & 0.01 & \\
\hline
\end{tabular}

Table 1: Combustion model candidates that are considered in the PEC-framework.

\section{Results}

\subsection{Reference simulation}

In this section, we will first establish a reference solution from the detailed chemistry simulation (DC). The performance of the skeletal chemistry (SC) as well as single-regime non-premixed (FPV) and premixed (FPI) flamelet models is then examined through direct comparisons with the detailed chemistry results. To obtain a quantitative assessment of the model accuracy, we define a global relative error for quantity $\alpha$ as

$$
e_{\alpha}=\frac{\left\|\alpha-\alpha^{\mathrm{DC}}\right\|_{1}}{\left\|\alpha^{\mathrm{DC}}\right\|_{1}},
$$

where $\|\cdot\|_{1}$ denotes the $L^{1}$-norm over the computational domain. The relative error for all models is listed in Table 2.

\subsubsection{Detailed chemistry}

To obtain a reference solution for the performance assessment of the PEC-formulation, a simulation with the detailed chemical kinetics model is performed. The results from this simulation are presented in the top row of Fig. 3, showing (from left to right) mixture fraction, temperature, and mass fractions of carbon monoxide $(\mathrm{CO})$ and nitric oxide $(\mathrm{NO})$.

The simulation results show that the flame is anchored at $x / L=0.25$, indicating that the PID-controller is able to stabilize the flame at a specified location. The inlet velocity for this condition is $U_{0}=59.1 \mathrm{~cm} / \mathrm{s}$, which is in good agreement with theoretical predictions [50]. Moreover, the stabilized flame remains at the same location even after the controller has been deactivated. With this inlet velocity, the Reynolds number based on $L, U_{0}$ and the viscosity of air at ambient condition is approximately 800 . At this condition, the flow is expected to be laminar.

The mixture fraction field, shown in the left column, indicates broadening of the mixture stratification within the flame. This can be attributed to dilatational effects of the flame, and such characteristics is also seen in previous work $[49,57]$. From the temperature field (second column), it can be seen that the maximum temperature is found along the centerline where the mixture is near stoichiometric condition. 


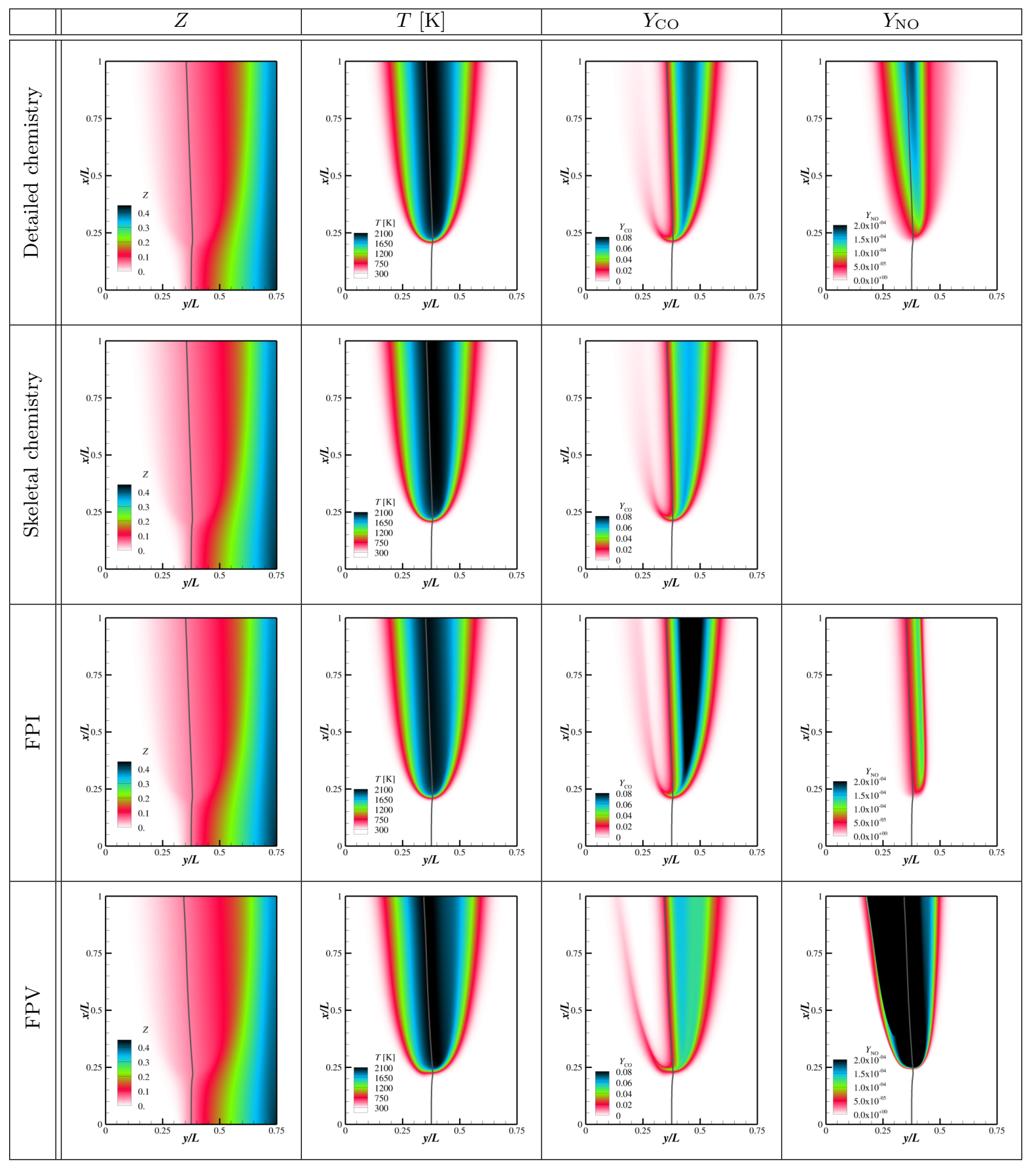

Figure 3: Mixture fraction $Z$, temperature $T$, and mass fractions of $\mathrm{CO}$ and $\mathrm{NO}$ for the tribrachial flame configuration calculated by detailed chemistry (DC, first row), skeletal chemistry (SC, second row) FPI (third row), and FPV (last row); stoichiometric mixture fraction is shown as a gray isoline. Note that the skeletal mechanism (SC) does not contain NO-chemistry.

\subsubsection{Skeletal chemistry}

A skeletal chemistry model (SC) derived from the GRI 1.2 mechanism is considered in this prior assessment. This skeletal mechanism will be considered as a chemistry-manifold submodel in subsequent PEC-simulations. The predicted mixture fraction, temperature and CO mass-fraction fields are shown in 


\begin{tabular}{|c||c|c|c|c|c|c|}
\hline Manifold Models & $e_{Z}[\%]$ & $e_{T}[\%]$ & $e_{Y_{\mathrm{CO}_{2}}}[\%]$ & $e_{Y_{\mathrm{H}_{2} \mathrm{O}}}[\%]$ & $e_{Y_{\mathrm{CO}}}[\%]$ & $e_{Y_{\mathrm{NO}}}[\%]$ \\
\hline \hline SC & 0.1 & 0.7 & 1.6 & 1.2 & 10.9 & - \\
\hline FPI & 0.3 & 1.7 & 7.8 & 2.2 & 26.8 & 66.2 \\
\hline FPV & 2.2 & 7.3 & 16.7 & 13.0 & 21.0 & 223.2 \\
\hline
\end{tabular}

Table 2: Comparisons of relative errors for simulations with skeletal chemistry (SC) and single-regime flamelet models (FPI and FPV) relative to detailed chemistry (DC) results.

the second row of Fig. 3. Note that the GRI 1.2 mechanism does not contain a description of the NO formation pathways and results for NO are therefore omitted. The predictions of mixture fraction and temperature are in good agreement with the detailed chemistry solution. The skeletal chemistry model tends to under-predict the peak $\mathrm{CO}$ mass fraction as compared to the detailed chemistry results.

Despite the qualitative similarity with the single-regime flamelet results, the quantitative comparison shown in Table 2 reveals that the skeletal chemistry model is generally more accurate than the flamelet models. This confirms that the higher computational requirement of the skeletal chemistry can result in a more accurate model. Therefore, this skeletal chemistry model is suitable to be included in the list of candidate combustion models. The advantages and disadvantages of including additional reduced chemistry models in the PEC-simulation will be examined in an a posteriori analysis in Sec. 5.2.

\subsubsection{Single-regime combustion models}

To establish a baseline solution for the single-regime combustion models, two separate simulations with the FPV and the FPI combustion models are performed. For both simulations, steady flamelet solutions are generated and tabulated as a function of mixture fraction $Z$ and reaction progress variable $C$. The progress variable is here defined as [58] $C=Y_{\mathrm{CO}_{2}}+Y_{\mathrm{CO}}+Y_{\mathrm{H}_{2} \mathrm{O}}+Y_{\mathrm{H}_{2}}$. Laminar diffusion flames are obtained from the solution of flamelet equations in mixture fraction space, spanning the entire S-shape curve. Freely propagating unstrained premixed flames for the range of flammable equivalence ratios are computed in physical space. Both chemistry tables use the same resolution with $200 \times 200$ grid points along the directions of $Z$ and $C$. Results from the FPV and FPI simulations are shown in the third and fourth rows of Fig. 3. This direct comparison shows that the mixture fraction and temperature are in good agreement with results from the detailed chemistry simulation. However, substantial differences are observed for CO and NO. In general, the FPI-model over-predicts the formation of CO while the FPV-model under-predicts $\mathrm{CO}$ on the fuel-rich side; an opposite trend is observed for predictions of NO. In this context it is noted that these discrepancies are expected since the reaction-transport manifolds are constructed form steady flamelet solutions without consideration of slow time-scale effects.

In general, the FPI-model is more accurate compared to the FPV-model for this configuration. The mixing process is well predicted by both models. As shown in the first column of Fig. 3, enhanced mixing can be observed at the flame location due to the dilatation, which is also well captured due to the accurate prediction of the temperature field by both manifold models. In addition to temperature, FPI also provides an accurate prediction for major species such as $\mathrm{CO}_{2}$ and $\mathrm{H}_{2} \mathrm{O}$, indicating that temperature and stable species are insensitive to the manifold representation. In contrast, the results confirm that both flamelet models exhibit deficiencies in accurately predicting intermediate species.

\subsubsection{Flame index and regime indicator}

The flame index due to Yamashita et al. [59] is often used as a combustion regime indicator to provide a characterization of the flame topology in different regions of the flame. This index is defined as:

$$
\mathrm{FI}=\frac{\nabla Y_{\mathrm{F}} \cdot \nabla Y_{\mathrm{O}}}{\left|\nabla Y_{\mathrm{F}}\right|\left|\nabla Y_{\mathrm{O}}\right|},
$$

where $Y_{\mathrm{F}}$ and $Y_{\mathrm{O}}$ represent mass fractions of fuel and oxidizer, respectively. The flame index is constructed such that a value of -1 indicates a non-premixed combustion regime, corresponding to a misalignment of the 
gradients of fuel and oxidizer, and a value of 1 signifies a premixed regime, where the respective gradients are aligned.

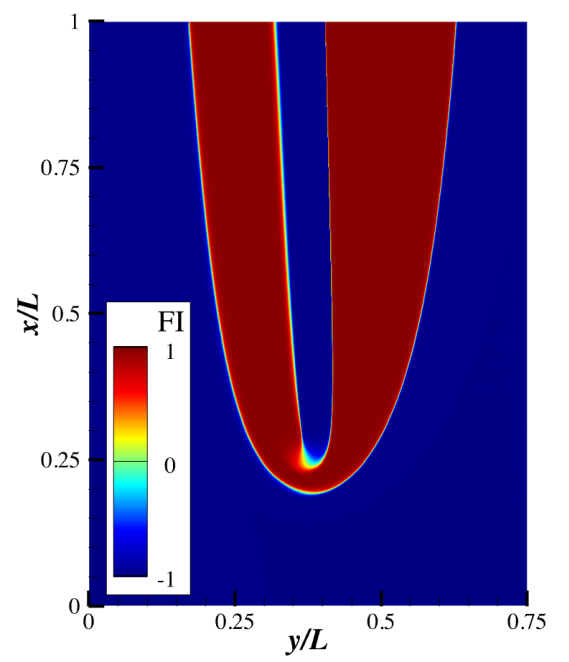

Figure 4: Yamashita flame index evaluated from the reference solution with detailed chemistry.

The Yamashita flame index is evaluated from the detailed chemistry simulation. The results are presented in Fig. 4, showing that this flame index captures the tribrachial flame structure in which a non-premixed flame is embedded between two premixed flame branches. The Yamashita index also suggests that both premixed and non-premixed branches are close to the asymptotic regimes and the area that is occupied by partially-premixed flames is very limited. Hence, one might expect that the error made by the single-regime flamelet models should mostly be in the region in which the Yamashita index does not match the regime of the flamelet model. To examine this, Fig. 5 provides quantitative comparisons of temperature and species profiles along the axial location $x / L=0.75$. In this figure, results from the detailed chemistry simulation are color-coded by the Yamashita index with FI $=-1$ (non-premixed) shown in blue and FI $=1$ in red (premixed). Under the condition that the flame index describes the flame topology, the detailed chemistry results should be close to the corresponding regime-specific flamelet results.

The temperature profile, shown in Fig. 5(a), is consistent with the flame index, showing that the FPVmodel is more accurate in the diffusion-flame region and simulations with the FPI-model are in better agreement with the detailed chemistry simulation in premixed flame branches. The analysis of the simulation results for $\mathrm{H}_{2} \mathrm{O}$ (Fig. 5(b)), shows that the FPI-model is consistently more accurate than the FPV-model, independent of the combustion regime given by the flame index. Comparisons for $\mathrm{CO}$, in Fig. 5(c), show that both models mispredict the peak of the CO-production. Furthermore, the FPV-model shows slightly better agreement with the detailed chemistry results on the fuel-rich side, whereas the FPI-model is more accurate on the diffusion-dominated fuel-lean side. Results for the mass fraction of NO are presented in Fig. 5(d), showing that - independent of the combustion regime - both flamelet models provide only an inadequate representation of the NO profile. The flame index is a global indicator that relies on information about the flame topology and does not exhibit any sensitivity to minor species. This suggests that the flame index can provide adequate guidance for the description of temperature and major species, but more selective criteria are necessary to accurately represent intermediate species and pollutants. Therefore, the applicability of flamelet models cannot be fully determined by a global regime indicator alone and should depend on the quantities of interest.

\subsection{Pareto-efficient combustion modeling results}

In this section, the performance of the PEC-approach is examined in detail. Specific focus of this investigation is on the evaluation of the dynamic model allocation, quantitative assessment of model accuracy 


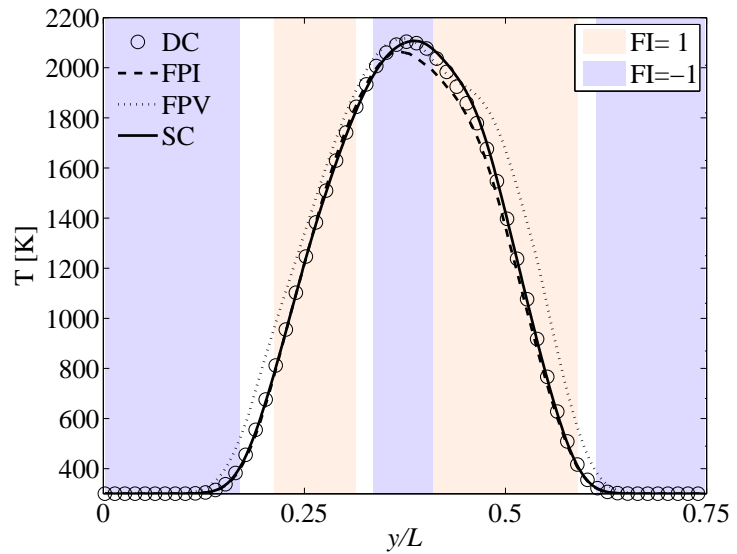

(a) Temperature.

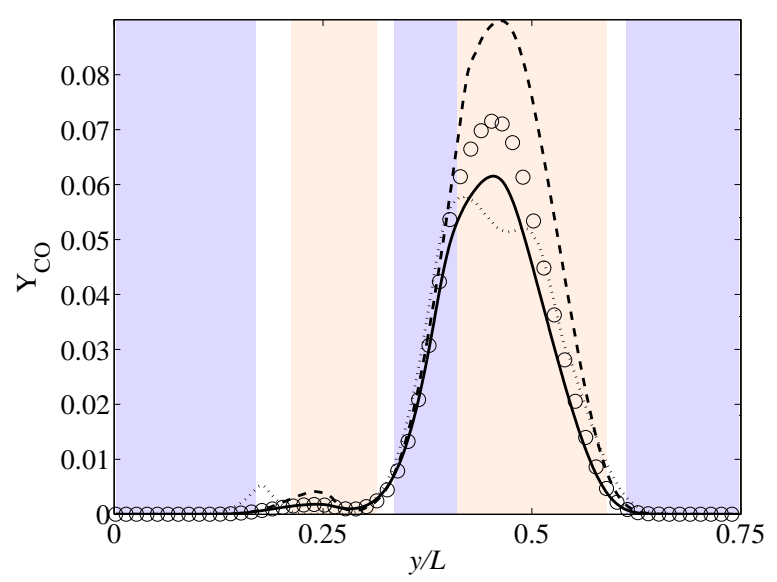

(c) Mass fraction of CO.

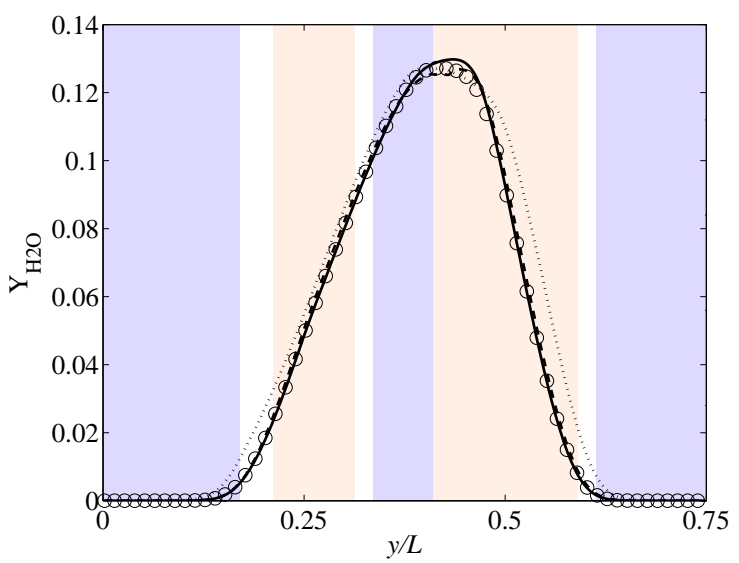

(b) Mass fraction of $\mathrm{H}_{2} \mathrm{O}$.

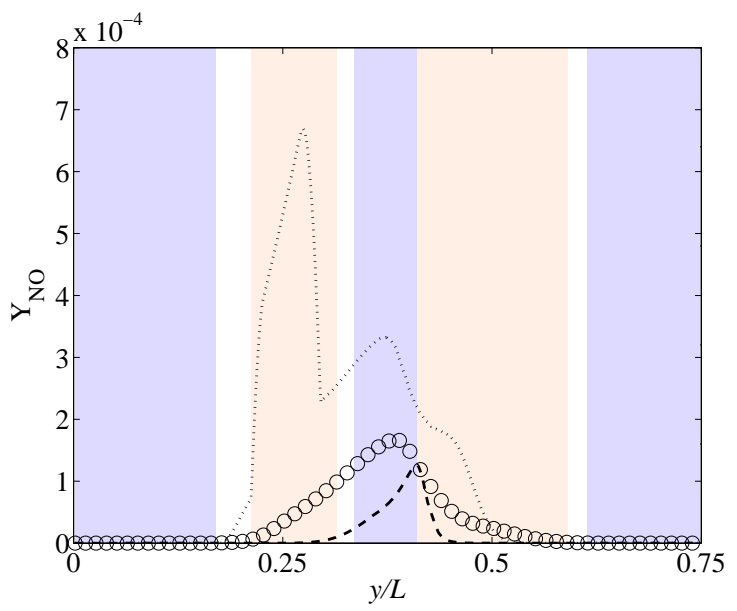

(d) Mass fraction of NO.

Figure 5: Comparison of profiles along axial cross section $x / L=0.75$ results for (a) temperature and mass fractions of (b) $\mathrm{H}_{2} \mathrm{O}$, (c) $\mathrm{CO}$ and (d) NO for detailed and skeletal chemistry models, and single-regime FPI and FPV combustion models.

through direct comparisons with detailed chemistry and single-regime flamelet models, and the sensitivity of the error threshold parameter, submodel-specification, and quantities of interest.

In this study, we consider the following sets of candidate combustion models:

$$
\begin{aligned}
& M_{1}=\{\mathrm{IM}, \mathrm{FPI}, \mathrm{FPV}, \mathrm{DC}\}, \\
& M_{2}=\{\mathrm{IM}, \mathrm{FPI}, \mathrm{FPV}, \mathrm{DC}, \mathrm{SC}\},
\end{aligned}
$$

two sets of quantities of interest,

$$
\begin{aligned}
& Q_{1}=\left\{Y_{\mathrm{CO}_{2}}, Y_{\mathrm{H}_{2} \mathrm{O}}, Y_{\mathrm{H}_{2}}, Y_{\mathrm{CO}}, Y_{\mathrm{NO}}\right\}, \\
& Q_{2}=\left\{Y_{\mathrm{CO}_{2}}, Y_{\mathrm{H}_{2} \mathrm{O}}, Y_{\mathrm{H}_{2}}, Y_{\mathrm{CO}}\right\},
\end{aligned}
$$

${ }_{641}$ and a range of values for the penalty parameter

$$
\lambda=\{0.002,0.01,0.02,0.1,0.2,1.0,2.0,10.0\} .
$$


Except for the discussion on the dynamic model assignment in Sec. 5.2.4, all results are obtained after three flow-through times from an initialization with the detailed chemistry solution. This provides faster convergence to the steady state solution as compared to using the premixed flamelet solution as initial condition. The initial model assignment is uniform and corresponds to the premixed flamelet (FPI) representation, so that only information about the velocity, the mixture fraction and the progress variable is used from the reference solution. The outcome of initializing with the premixed flamelet solution is further examined in the discussion on the dynamic model allocation.

As discussed in Sec. 3.7, PEC-simulations are inherently dynamic. Therefore, PEC-calculations with submodel updates at each iteration were performed until a steady-state solution for $m$ is obtained. In this section, PEC-simulations are first quantitatively assessed using results obtained from the converged solution. The dynamic behavior of the PEC-framework is then examined in detail in Secs. 5.2.4 and 5.2.5.

The drift from the manifold for all reaction-transport models is evaluated from Eq. (12), for which the Jacobian $\partial \widehat{\phi} / \partial \boldsymbol{\psi}$ is pre-tabulated for all quantities of interest, and tabulated in the chemistry-libraries. However, the drift from the manifold for the skeletal chemistry model requires the evaluation of detailed chemistry source terms. Therefore, $M_{1}$ is considered as the baseline set of model candidates as it excludes the skeletal chemistry model.

\subsubsection{Tradeoff between accuracy and efficiency}

We begin the analysis of the PEC-model by discussing the model accuracy. The set of candidate combustion models used in this study is $M_{1}$ and $Q_{1}$ is used for the quantities of interest.

Results of the simulation for three different values of $\lambda$ are shown in Fig. 6 . To facilitate a direct comparison with the detailed chemistry simulation, we also include these results in the first row. In this context it is noteworthy to mention that detailed chemistry results would be obtained by setting $\lambda \rightarrow 0$ in PEC.

A comparison of the temperature field is presented in the first column of Fig. 6, and it can be seen that the temperature is insensitive to the threshold value $\lambda$. This good agreement for the temperature is expected since similar agreement was obtained from the single-regime flamelet models in Fig. 3. In contrast, PEC-predictions for CO, shown in the second column of Fig. 6, are significantly improved compared to the results obtained with the single-regime combustion models. This is due to the local utilization of the detailed-chemistry description in topologically complex regions that are not adequately described by reaction-transport manifold models. With increasing penalty, the accuracy for the CO-prediction gracefully deteriorates. A similar trend is observed for predictions of NO, which are presented in the third column of Fig. 6. It can be seen that rather small constraints on $\lambda$ are necessary to ensure an accurate prediction of NO, and the PEC-model error rapidly increases for $\lambda \geq 0.2$.

The model assignments for the three different PEC-calculations are illustrated in the last column of Fig. 6. The submodel assignment conforms to the model characterization that was discussed in Sec. 5.1. Independent of the value of the penalty, the inert mixing model covers approximately $50 \%$ of the domain, while the subdomain that is described by the FPI-model increases from $12 \%$ for the case with $\lambda=0.02$ to $40 \%$ for $\lambda=0.2$ and larger values. This model assignment is consistent with results from the singleregime model analysis, indicating that the premixed model provides a better agreement with the detailed chemistry results. For the smallest value of $\lambda$, a substantial region of the domain (36\%) is covered by the detailed chemistry simulation, providing accurate simulation results yet at increased computational cost. In this region none of the reaction-transport manifold models provides an accurate prediction of $Q$, so that the PEC-procedure seeks to maximize the utilization of the detailed chemistry model. Interestingly, it can be seen that for large values of $\lambda$ a portion of the fuel-rich premixed flame branch is represented by the FPV-model (shown in red).

To examine the performance of the PEC-framework further, we vary the penalty $\lambda$ between $2 \times 10^{-3}$ and 10, and use $Q_{1}$ as quantities of interest. Results for the relative error (normalized with respect to the detailed chemistry results from Sec. 5.1.1) for $T, Y_{\mathrm{CO}}$ and $Y_{\mathrm{NO}}$, model assignment, and the estimated cost are presented as a function of $\lambda$ in Fig. 7. An analysis of the relative error (Fig. 7(a)) shows that for the smallest value of $\lambda$, all quantities of interest are predicted accurately within less than $1 \%$ relative error. 


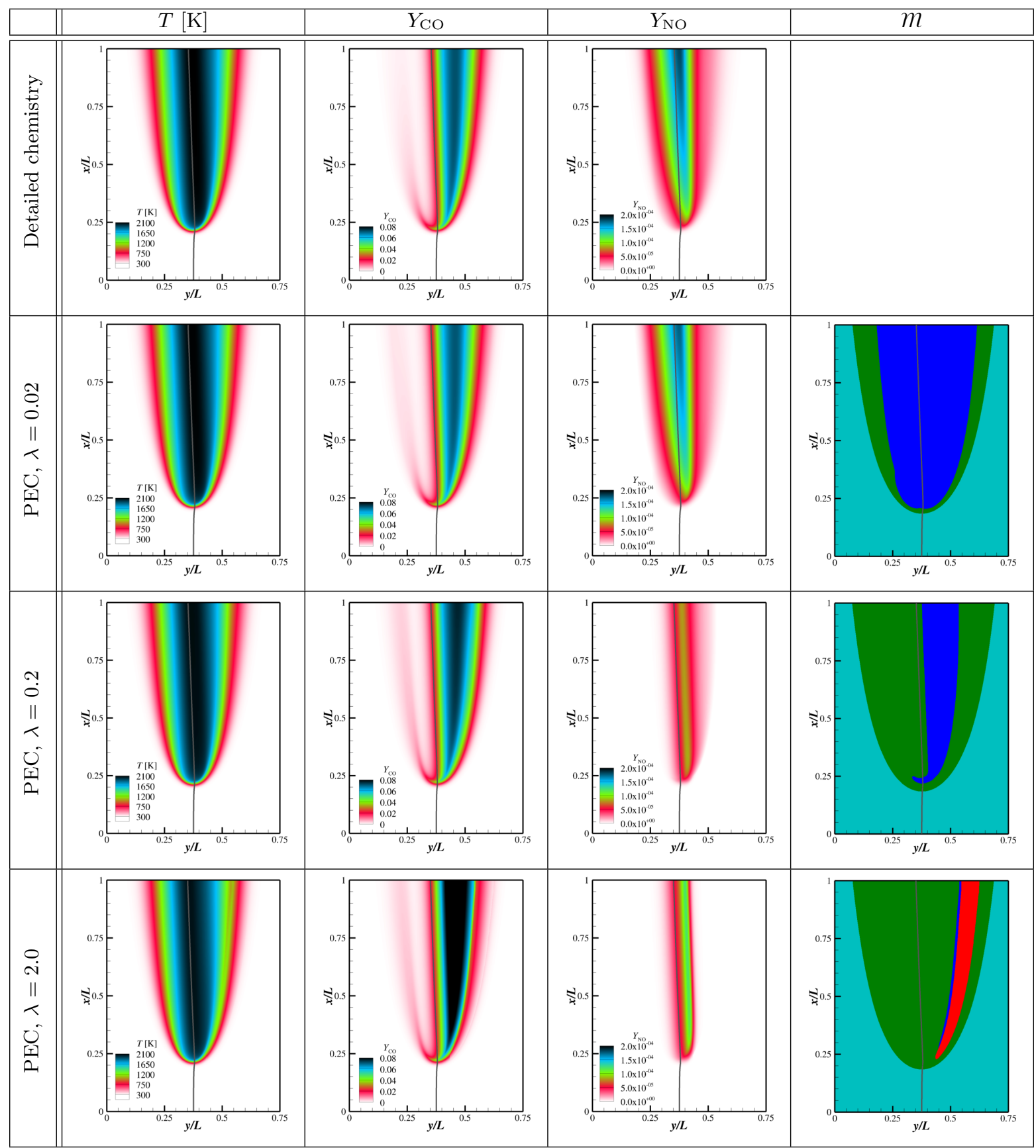

Figure 6: Results from PEC-model, showing temperature, mass fractions of CO and NO, and model assignment $m$ for detailed chemistry simulation (first row) and PEC-simulations with $\lambda=0.02$ (second row), $\lambda=0.2$ (third row), and $\lambda=2$ (last row); stoichiometric mixture fraction is shown as a gray isoline. Model assignment is: IM (cyan), FPI (green), FPV (red), and DC (blue).

With increasing $\lambda$, the error for the different quantities increases, and - dependent on the QoI $-e_{\alpha}$ levels off at values that are comparable to the results of the single-regime FPI-model. Interestingly, at the largest value for $\lambda, Y_{\mathrm{CO}}$ predicted by PEC is slightly more accurate than that from the FPI-simulation. This can be attributed to the utilization of the FPV-model at appropriate locations (Fig. 7(b)). The prediction of $Y_{\text {NO }}$ by the PEC-model is comparable to that of the FPI simulation as the utilization of the detailed chemistry 


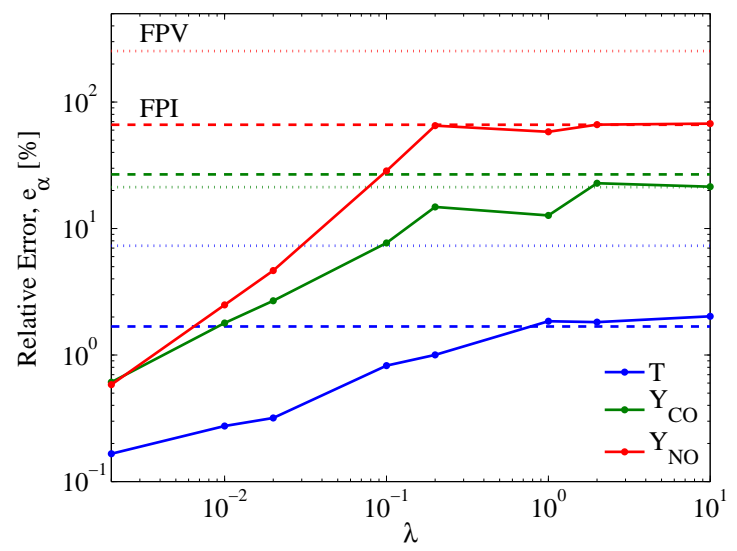

(a) Relative error.

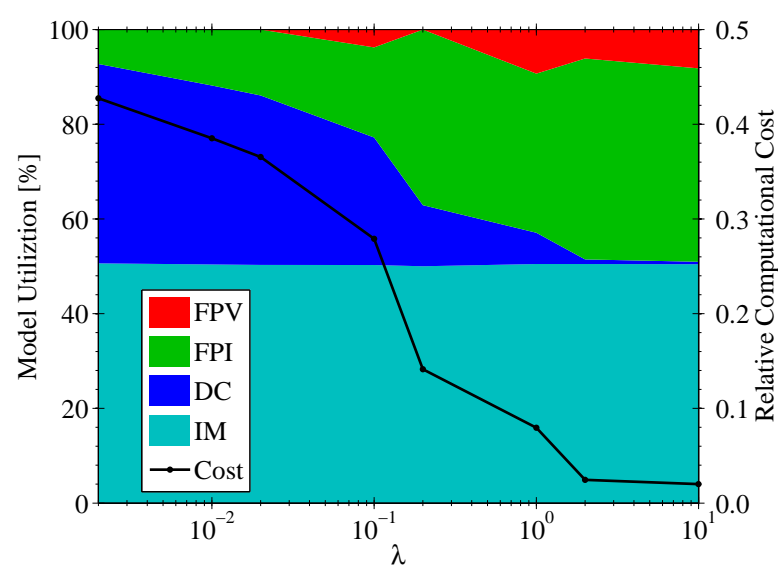

(b) Model assignment and estimated cost.

Figure 7: PEC-results as a function of the penalty term $\lambda$, showing (a) relative error of species mass fractions and temperature, and (b) model assignment and estimated cost; quantities of interest is defined as $Q_{1}=\left\{Y_{\mathrm{CO}_{2}}, Y_{\mathrm{H}_{2} \mathrm{O}}, Y_{\mathrm{H}_{2}}, Y_{\mathrm{CO}}, Y_{\mathrm{NO}}\right\}$ and the set of candidate models is $M_{1}=$ IIM, FPI, FPV, DC $\}$. Dashed lines indicate FPI-results and dotted lines indicate FPV-results, both being independent of $\lambda$.

model is reduced at large values of $\lambda$. It is noteworthy to point out that the PEC-simulation, at large penalty values, predicts the temperature-field with a slightly larger error than the single-regime FPI-model. This can be rationalized by the fact that the temperature is not included in the set of quantities of interests, and the PEC-procedure does not seek to reduce this error.

Figure 7(b) shows the assignment of each candidate model as a function of $\lambda$. As anticipated, the adaptive model assignment shifts toward flamelet models with increasing $\lambda$, corresponding to reduced accuracy requirement and cost. The usage of the detailed chemistry model decreases from $40 \%$ to zero while that of the FPI-model increases from less than $10 \%$ to $40 \%$. As a result of the tribrachial shape of the flame, the inert mixing model consistently occupies $50 \%$ of the domain. The usage of the FPV model never exceeds $10 \%$ which confirms the fact that the FPV-model is not suitable for this configuration.

As the usage of the detailed chemistry model decreases with increasing $\lambda$, the computational cost reduces as shown in Fig. 7(b). Note that the estimated cost is normalized by the cost of the full detailed chemistry simulation. The PEC approach is theoretically able to operate at the computational costs that is 30 times smaller. However, the full utilization of this cost saving requires dynamic load balancing. A combination of PEC and adaptive mesh refinement would further reduce the cost by locally refining the mesh in regions where the detailed chemistry model is used. Such extensions can be accommodated by the PEC-model.

\subsubsection{Quantities of interest}

The set of quantities of interest utilized in the previous study contains the pollutant species NO, and accurate prediction of this chemical species is only achieved for the given set of candidate models by reducing the penalty values. TO confirm this, we will next examine the performance of PEC with different sets of $Q$. For this, we exclude $\mathrm{NO}$, and consider the quantities of interest $Q_{2}$ while keeping $M_{1}$ as the set of candidate models.

Results obtained from the PEC-simulation with $\lambda=0.02$ are shown in Fig. 8. Comparisons with the results in Fig. 6 for the same value of $\lambda$ show qualitative and quantitative differences in the model assignment and NO-prediction. Unlike the previous case where the detailed chemistry representation is used in the entirety of the flame, a small region on the fuel-lean side is now represented using flamelet models with this specification of the quantities of interest. Interestingly, the FPV-model is utilized while the PEC-computation with $Q_{1}$ only considers FPI from the set of reaction-transport manifold models. The FPV-model is assigned to a region where the Yamashita flame index indicates a non-premixed flame topology. 


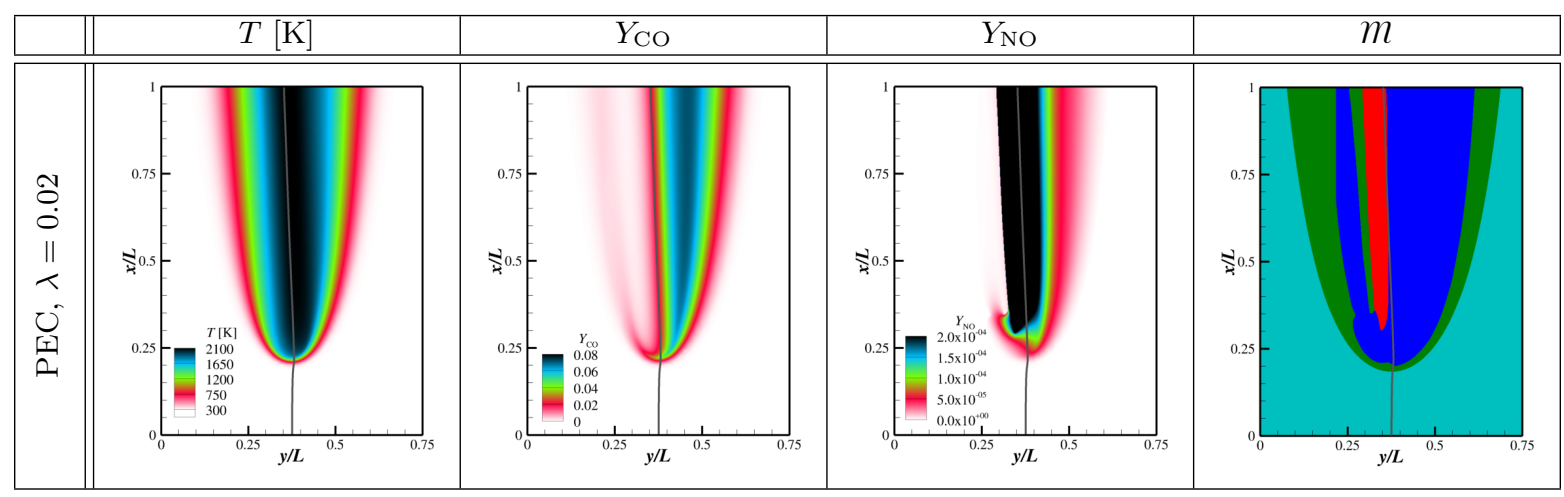

Figure 8: Temperature, mass fractions of $\mathrm{CO}, \mathrm{NO}$, and model assignment $m$ for PEC-simulation of $\lambda=0.02, M_{1}=$ $\{\mathrm{IM}, \mathrm{FPI}, \mathrm{FPV}, \mathrm{DC}\}$ and $Q_{2}=\left\{Y_{\mathrm{CO}_{2}}, Y_{\mathrm{H}_{2} \mathrm{O}}, Y_{\mathrm{H}_{2}}, Y_{\mathrm{CO}}\right\}$. Model assignment is: IM (cyan), FPI (green), FPV (red), and DC (blue).

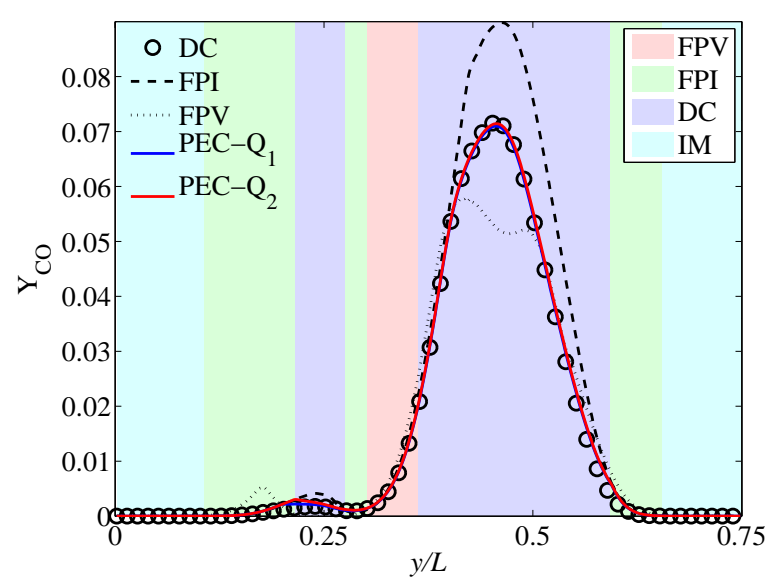

(a) CO mass fraction.

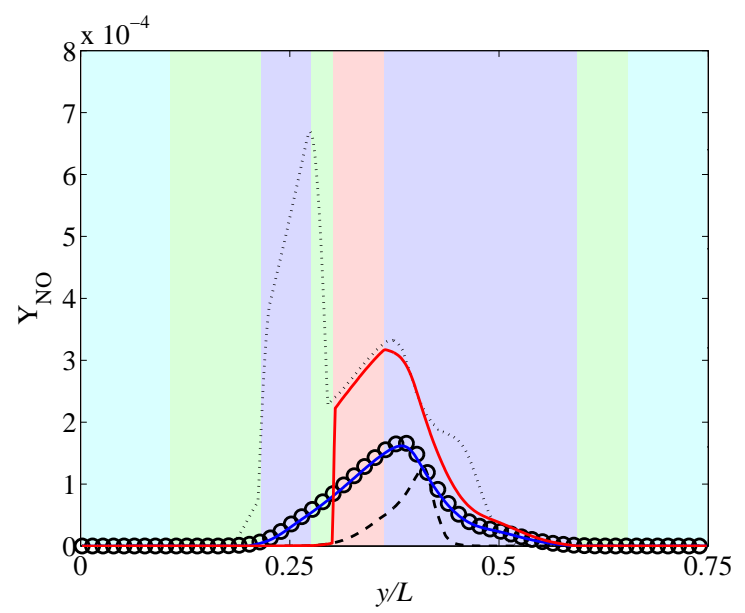

(b) NO mass fraction.

Figure 9: Profiles of $Y_{\mathrm{CO}}$ and $Y_{\mathrm{NO}}$ at $x / L=0.75$ for PEC-simulations with $\lambda=0.02$ and $M_{1}=\{\mathrm{IM}, \mathrm{FPI}, \mathrm{FPV}, \mathrm{DC}\}$ for $Q_{1}=\left\{Y_{\mathrm{CO}_{2}}, Y_{\mathrm{H}_{2} \mathrm{O}}, Y_{\mathrm{H}_{2}}, Y_{\mathrm{CO}}, Y_{\mathrm{NO}}\right\}$ and $Q_{2}=\left\{Y_{\mathrm{CO}_{2}}, Y_{\mathrm{H}_{2} \mathrm{O}}, Y_{\mathrm{H}_{2}}, Y_{\mathrm{CO}}\right\}$

Despite the differences in the model assignment, predictions for temperature and $Y_{\mathrm{CO}}$ are similar for both PEC-simulations. This agreement is confirmed by Fig. 9(a), showing comparison of $Y_{\mathrm{CO}}$-profiles at the axial location of $x / L=0.75$. For both simulations, the detailed chemistry model is utilized in regions where flamelet representations are not able to accurately predict $Y_{\mathrm{CO}}$. In contrast, Fig. 9(b) shows substantial discrepancies for the predictions of $Y_{\mathrm{NO}}$ using $Q_{2}$ as set of quantities of interest. The reason for the overprediction of NO for $0.3 \leq y / L \leq 0.5$ is the utilization of the FPV-manifold. Since NO is not included in the definition of $Q_{2}$, the model assignment procedure does not attempt to lower the error for this quantity.

This analysis demonstrates the importance of the selection of $Q$ on the accuracy of the PEC-results. As such, the inclusion of certain species in $Q$ provide direct user-control on the model accuracy. The implication of using different quantities of interest on the model accuracy is illustrated in Fig. 10, showing the relative error for $T, Y_{\mathrm{CO}}$ and $Y_{\mathrm{NO}}$ as a function of the estimated cost for $Q_{1}$ and $Q_{2}$. For moderate values of $\lambda$, the relative errors in $Y_{\mathrm{CO}}$ and temperature predicted with $Q_{2}$ are generally lower than that of $Q_{1}$ at the same estimated cost. This can be explained by the fact that the utilization of the detailed chemistry model in the simulation with $Q_{2}$ is less aggressive compared to the case with $Q_{1}$, and the premixed manifold-model 


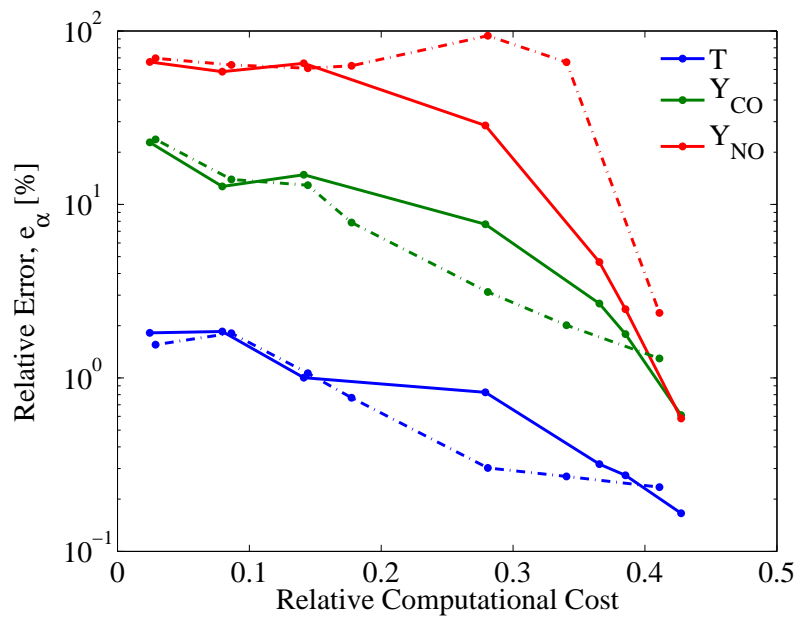

Figure 10: Pareto front, showing relative model error as a function of estimated cost for $Q_{1}=\left\{Y_{\mathrm{CO}_{2}}, Y_{\mathrm{H}_{2} \mathrm{O}}, Y_{\mathrm{H}_{2}}, Y_{\mathrm{CO}}, Y_{\mathrm{NO}}\right\}$ (solid line) and $Q_{2}=\left\{Y_{\mathrm{CO}_{2}}, Y_{\mathrm{H}_{2} \mathrm{O}}, Y_{\mathrm{H}_{2}}, Y_{\mathrm{CO}}\right\}$ (dashed line) with model assignment $M_{1}$.

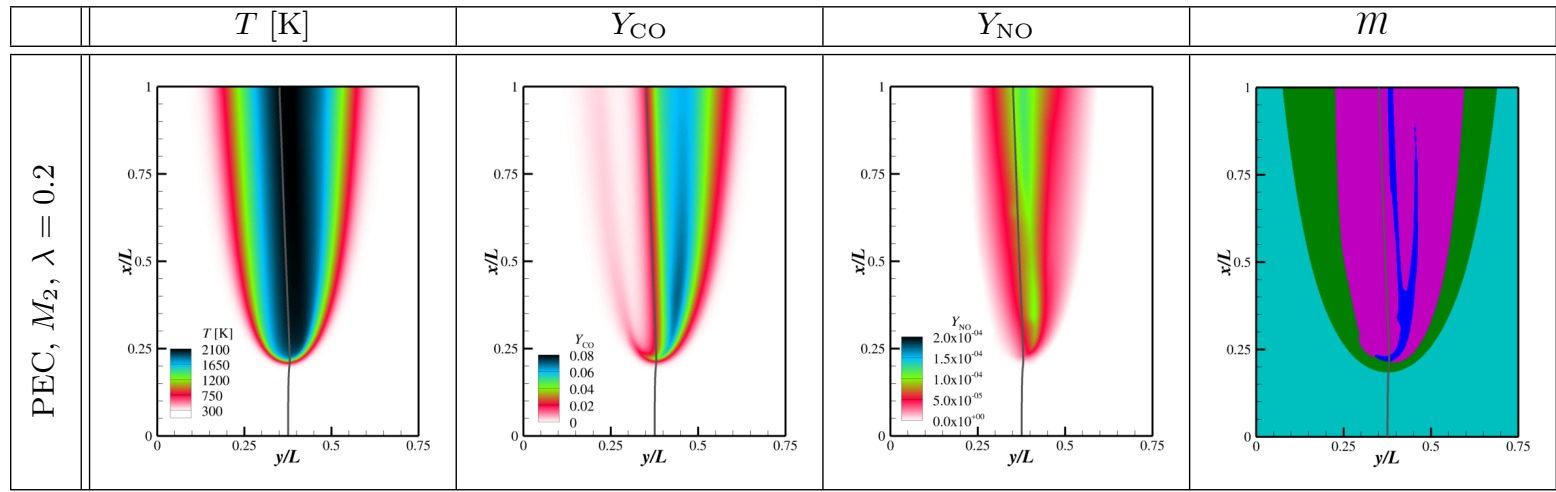

Figure 11: Temperature, mass fractions of $\mathrm{CO}, \mathrm{NO}$ and model assignment $m$ for PEC-simulation with $M_{2}=$ $\{\mathrm{IM}, \mathrm{FPI}, \mathrm{FPV}, \mathrm{DC}, \mathrm{SC}\}, Q_{1}=\left\{Y_{\mathrm{CO}_{2}}, Y_{\mathrm{H}_{2} \mathrm{O}}, Y_{\mathrm{H}_{2}}, Y_{\mathrm{CO}}, Y_{\mathrm{NO}}\right\}$ and $\lambda=0.2$; stoichiometric mixture fraction is shown as a gray isoline. Model assignment is: IM (cyan), FPI (green), SC (magenta), and DC (blue).

is adequate for predicting temperature and CO-mass fraction.

\subsubsection{Consideration of reaction-transport and chemistry manifolds}

The PEC-framework is designed to accommodate a large variety of combustion models. It is not only limited to reaction-transport manifolds and the detailed chemistry model that are considered in the previous two sections. To demonstrate this, we now also include a model from the class of skeletal chemistry manifolds into the set of candidate models, so that the set of candidate models is described by $M_{2}$ in Eq. 26b.

A calculation with $Q_{1}$ and $\lambda=0.2$ is performed, and the results of this simulation are shown in Fig. 11. The model assignment, shown in Fig. 11, clearly indicates that the skeletal chemistry model occupies the largest area within the reacted region of the simulation. In contrast, the model assignment for the simulation with $M_{1}$ and $\lambda=0.2$, shown in Fig. 6 , indicates the greatest utilization of premixed manifold model in the same reacted region. As mentioned in Appendix Appendix C, $\mathscr{D}$ of the skeletal chemistry model is generally smaller than that of the reaction-transport models. Therefore, it is expected that the skeletal chemistry model is favored over flamelet models in this new PEC-simulation.

The predicted $Y_{\text {NO }}$ field (Fig. 11) shows reasonable agreement with the detailed chemistry simulation results. This might be surprising as the skeletal chemistry model does not describe the formation of NO. 
Since, however the transport of $Y_{\mathrm{NO}}$ is solved for in regions where the skeletal chemistry model is utilized in PEC-simulations, it is important to recognize that an accurate prediction of $Y_{\mathrm{NO}}$ is possible if the region of NO-formation is described by a model of higher fidelity. This is the case here where the detailed chemistry model is utilized in regions of non-vanishing NO-production.

As a consequence of using the different set of candidate models, the current simulation yields predictions of $Y_{\mathrm{CO}}$ and $Y_{\mathrm{NO}}$ that are different from the results in Sec. 5.2.1. These differences are illustrated in Fig. 12, showing comparisons of radial profiles for these species at specific axial locations. At $x / L=0.5$, the predicted $Y_{\mathrm{CO}}$ profile for the current simulation clearly shows better agreement with detailed chemistry simulation results than simulations with $M_{1}$ and comparable $\lambda$. Specifically, the small peak in the $Y_{\mathrm{CO}}$ profile at $y / L \approx 0.25$ is more accurately captured by the simulation using $M_{2}$. Everywhere else, the predicted $Y_{\mathrm{CO}}$ profile is not worse than the simulation results with $M_{1}$ and $\lambda=0.2$. Further downstream at $x / L=0.75$, the peak value of $Y_{\mathrm{CO}}$ is slightly under-predicted. This is due to the utilization of the skeletal chemistry model, which tends to underpedict $Y_{\mathrm{CO}}$ in this region of the flame.

At both axial locations, shown in Fig. 12, the current simulation predicts a $Y_{\text {NO-profile that gradually }}$ decays near $y / L \approx 0.2$. This is in better agreement with the detailed chemistry simulation than the

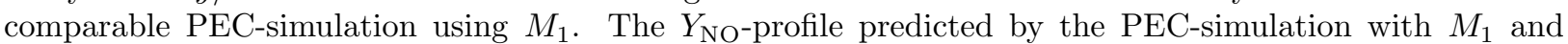
$\lambda=0.2$ displays a faster decrease of $Y_{\mathrm{NO}}$ at $y / L \approx 0.3$, and this is not observed in the detailed chemistry simulation results. Further downstream at $x / L=0.75$, the current simulation clearly predicts a $Y_{\text {NO }}$ profile that shows better agreement with the detailed chemistry simulation than the comparable simulation with $M_{1}$.

The examination of the results of the PEC-simulation with $M_{2}$ indicates that its prediction is comparable to that with $M_{1}$. To further investigate the model prediction tendencies associated with $M_{2}$, we consider relative errors in the prediction of quantities of interest for different values of $\lambda$. The evaluated relative errors for temperature, $Y_{\mathrm{CO}}$ and $Y_{\mathrm{NO}}$ as a function of estimated computational cost are shown in Fig. 13(a). The result confirms that the inclusion of the skeletal chemistry model can further improve the Pareto front compared to the PEC-simulations using $M_{1}$. However, the error for the prediction of NO is slightly higher than the error of the comparable $M_{2}$ simulation at high values of $\lambda$. At large values of $\lambda$, as shown in Fig. 13(b), the skeletal chemistry model is utilized without the aid of the detailed chemistry model. Since the skeletal chemistry model does not contain the reactions for $Y_{\mathrm{NO}}$, this quantity cannot be accurately predicted.

\subsubsection{Dynamic model assignment}

In the previous discussion on the flexibility of the PEC-framework, the results are obtained from simulations that were initialized from the detailed chemistry solution. This is done to ensure fast convergence to the steady state solution. However, the detailed chemistry solution is rarely available in most practical configurations. To demonstrate that the application of the PEC-framework is robust and convergence does not depend on an accurate initial solution, we consider a PEC-simulation that is initialized from the solution of the FPI-simulation. In this study, we select $M_{2}, Q_{2}$, and $\lambda=0.02$ as PEC-input parameters.

The time history of the model assignment of the PEC-simulation is illustrated in Fig. 14 and the model assignment at different time instances is shown in Fig. 15. Initially, only the inert mixing model and the FPI-model are utilized in the simulation. Within less than $5 \%$ of a convective time scale, the FPI model is replaced by the detailed chemistry model in the fuel rich region near the centerline, extending from the flame tip to the end of the simulation domain. At approximately $10 \%$ of a convective time scale, the region of the detailed chemistry model is mostly stabilized and it is surrounded by a region that is described by the skeletal chemistry model (Fig. 15(c)). The area occupied by the skeletal chemistry model is slowly growing in the fuel lean region, thereby successively replacing the FPI-model. For $t \geq L / U_{0}$, the model assignment reaches a steady state. That is expected since changes in the flow field can take about one convective time scale to fully propagate throughout the simulation domain. This shows that the model assignment procedure is a dynamic process and can equally be applied to unsteady calculations.

During the transition to the steady state solution, the overall agreement of the PEC-simulation with the reference solution improves continuously. This is illustrated in Fig. 14, showing the evolution of the error, which is normalized with the corresponding value at $t=0$, i.e. $e_{\alpha}(t) / e_{\alpha}(0)$. Since the initial condition is the 


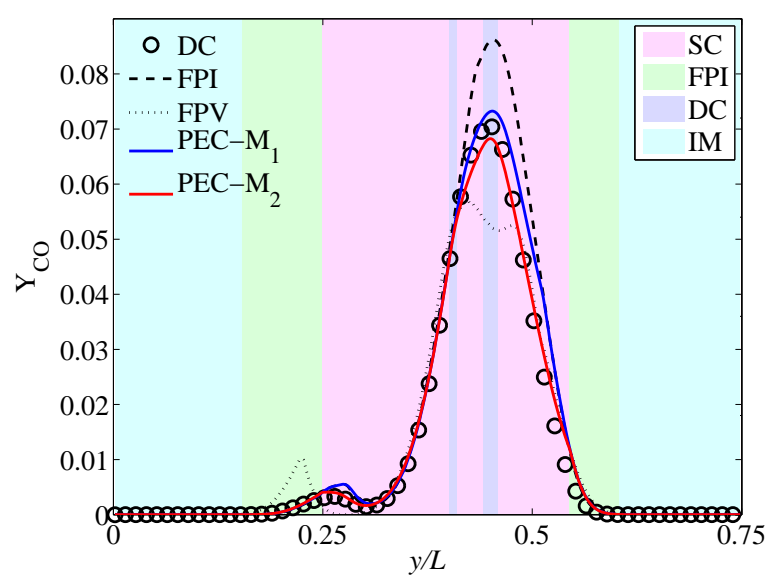

(a) CO-mass fraction at $x / L=0.5$.

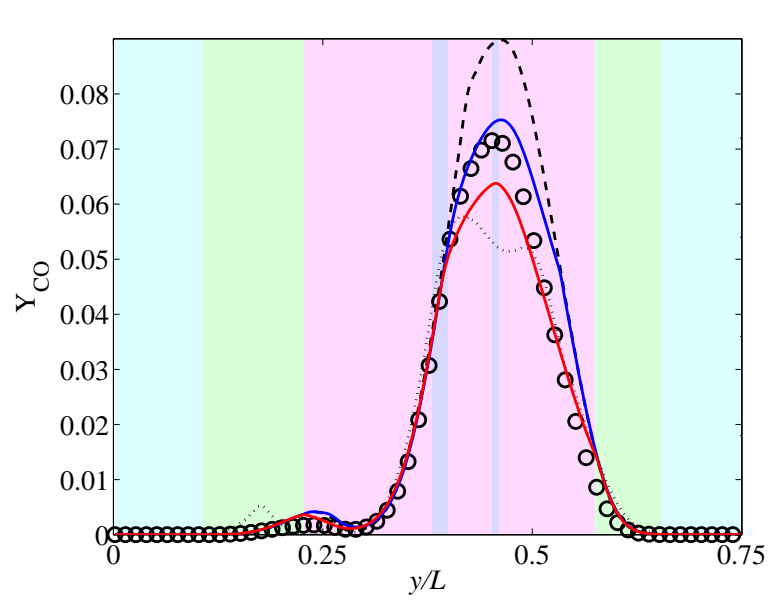

(c) CO-mass fraction at $x / L=0.75$.

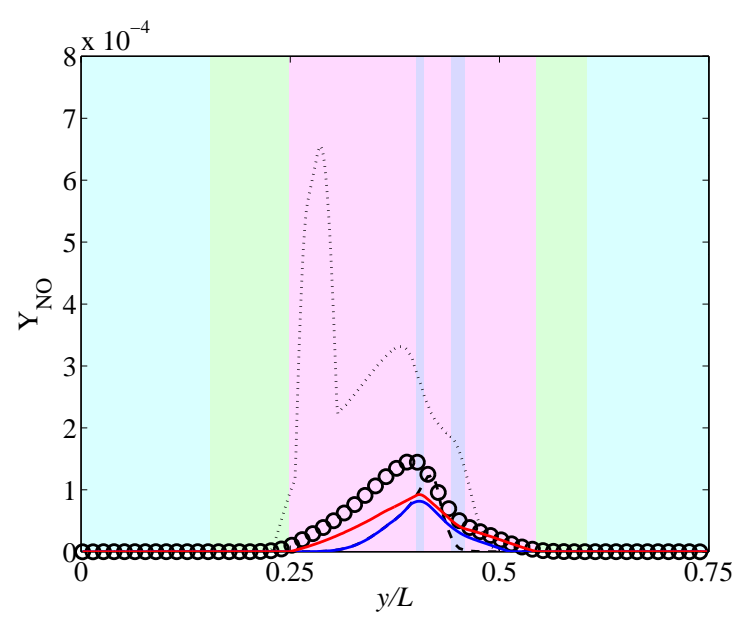

(b) NO-mass fraction at $x / L=0.5$.

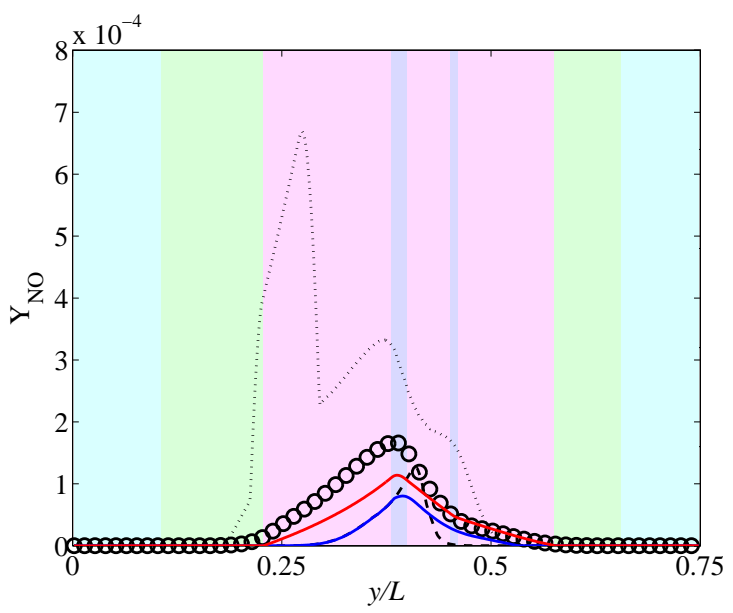

(d) NO massfraction at $x / L=0.75$.

Figure 12: Comparison of the profiles of $(\mathrm{a}, \mathrm{c}) Y_{\mathrm{CO}}$ and $(\mathrm{b}, \mathrm{d}) Y_{\mathrm{NO}}$ at $x / L=0.5$ (top) and 0.75 (bottom) for PECsimulations with $\lambda=0.2$ and $Q_{1}=\left\{Y_{\mathrm{CO}_{2}}, Y_{\mathrm{H}_{2} \mathrm{O}}, Y_{\mathrm{H}_{2}}, Y_{\mathrm{CO}}, Y_{\mathrm{NO}}\right\}$ under different $M_{1}=\{\mathrm{IM}, \mathrm{FPI}, \mathrm{FPV}, \mathrm{DC}\}$ and $M_{2}=$ $\{\mathrm{IM}, \mathrm{FPI}, \mathrm{FPV}, \mathrm{DC}, \mathrm{SC}\}$.

steady FPI result, this error is an indicator of the increase of accuracy with respect to the FPI calculation. In this case, the relative errors for $Y_{\mathrm{CO}}$ and $Y_{\mathrm{CO}_{2}}$ have decrease by $80 \%$ over the course of the transient process.

\subsubsection{Transient calculations}

Previous sections examined the PEC-performance using converged solution results, without considering the transient dynamics in adjusting the model assignment. To further evaluate the dynamic behavior of the PEC-framework, in the following we consider a series of PEC-simulations with unsteady inlet conditions. The inflow profile for the velocity is obtained from a synthetic $2 \mathrm{D}$ turbulence spectrum, whose mean streamwise velocity is set to a value of $60.0 \mathrm{~cm} / \mathrm{s}$. The scalar inflow profile remains the same as for all previous cases. In this study, we selected $M_{1}$ and $Q_{1}$ as PEC-input parameters. The initial condition is obtained from the corresponding steady-state calculation with $\lambda=0.2$, which is shown in the third row of Fig. 6 .

To evaluate the performance of the PEC-framework in describing the dynamics of the flame, we plot the temporal evolution of the flame tip location in Fig. 16. The location of the flame tip is identified as the 


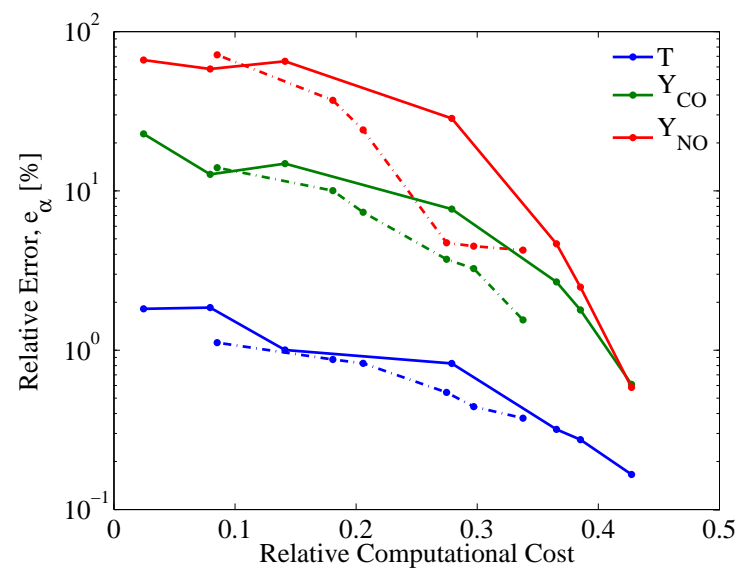

(a) Pareto front.

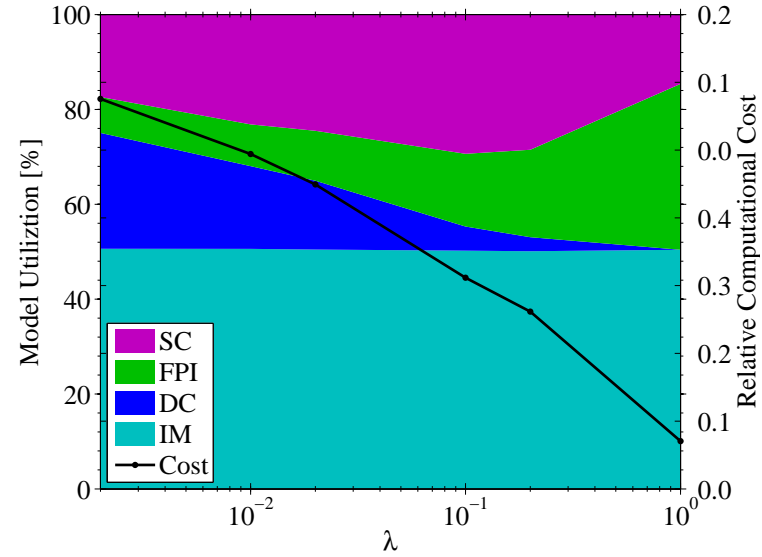

(b) Model assignment and estimated cost.

Figure 13: (a) Pareto front, showing relative model error as a function of estimated cost for PEC-simulations with $Q_{1}=$ $\left\{Y_{\mathrm{CO}_{2}}, Y_{\mathrm{H}_{2} \mathrm{O}}, Y_{\mathrm{H}_{2}}, Y_{\mathrm{CO}}, Y_{\mathrm{NO}}\right\}$ for $M_{1}=\{\mathrm{IM}, \mathrm{FPI}, \mathrm{FPV}, \mathrm{DC}\}$ (solid line) and $M_{2}=\{\mathrm{IM}, \mathrm{FPI}, \mathrm{FPV}, \mathrm{DC}, \mathrm{SC}\}$ (dashed dotted line); (b) model assignment and estimated cost for PEC-simulation with $M_{2}$ and $Q_{1}$.

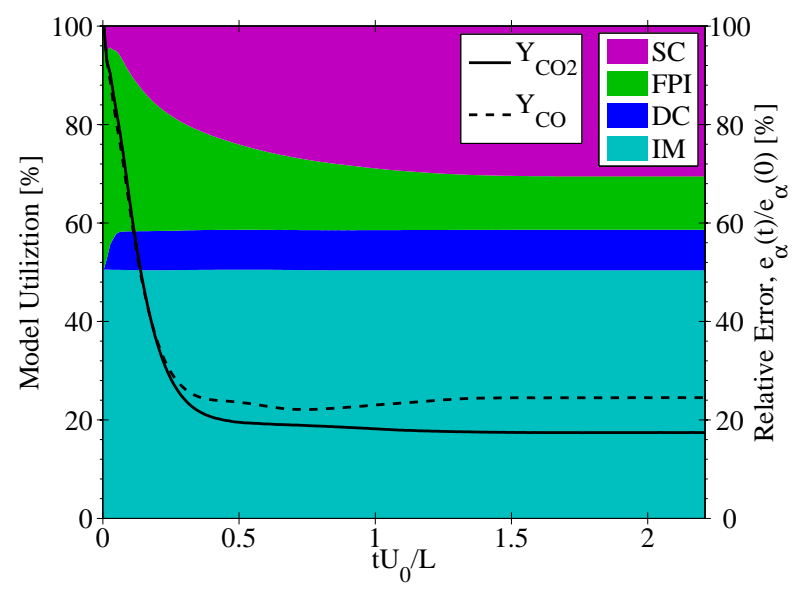

Figure 14: Temporal evolution of model assignment breakdown and relative reduction in error for the mass fractions of $\mathrm{CO}_{2}$ and CO for the PEC-simulation utilizing $M_{2}, Q_{2}$ and $\lambda=0.02$.

most upstream point along the isoline of $T=400 \mathrm{~K}$. Results that correspond to five different values of $\lambda$ are shown in Fig. 16, where $\lambda=0$ corresponds to a detailed chemistry simulation. The same observation as in Sec. 5.2.1 can be made that the accuracy of the solution can be increased by reducing the value of $\lambda$, so that the PEC-calculation will converge to the detailed chemistry simulation as $\lambda \rightarrow 0$. In general, the calculations utilizing a larger region with FPI-model assignment have a slower response and a slower flame speed. Due to the non-linear nature of the system, the discrepancy increases over time so that the solution slowly diverges. A more quantitative analysis for the divergence of the flame-tip location is illustrated in Fig. 17, showing the distance between the prediction from simulations with different values of $\lambda$ and the reference case with $\lambda=0$ (detailed chemistry result). The initial linear increment of the distance can be attributed to a consistent level of under-prediction for the flame speed. At later times, this effect is compounded by interaction of the flame with the unsteady flow field, thereby further modifying the trajectories of the flame movement and amplifying the error.

Since the model assignment, $m$, is dynamically updated at each iteration, it reacts to the transient 


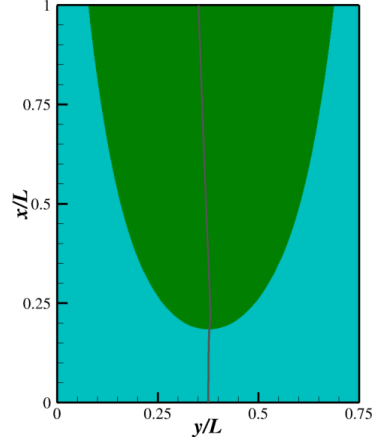

(a) $t U_{0} / L=0$.

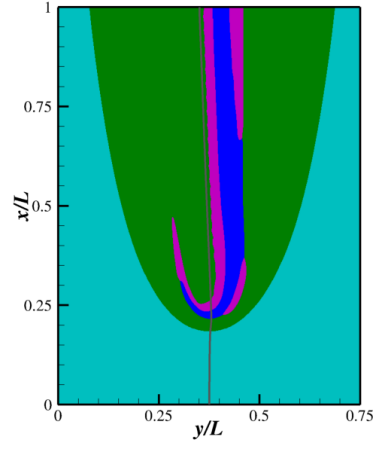

(b) $t U_{0} / L=0.03$.

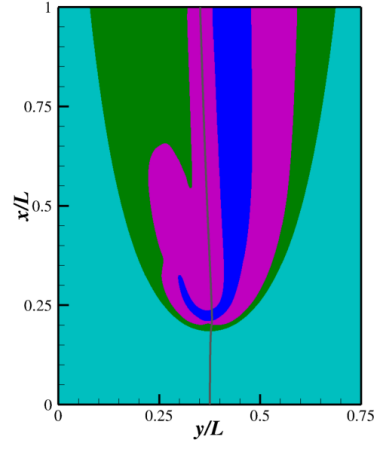

(c) $t U_{0} / L=0.35$.

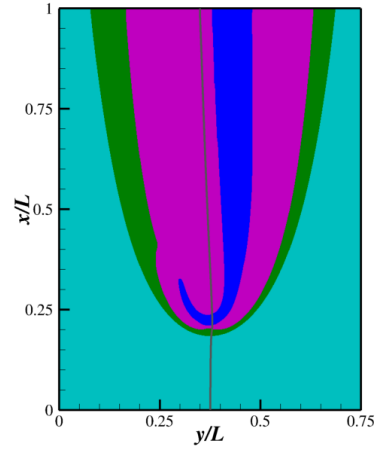

(d) $t U_{0} / L=2.00$.

Figure 15: Model assignment at selected times for the PEC-simulation utilizing $M_{2}, Q_{2}$ and $\lambda=0.02$. Model assignment is: IM (cyan), FPI (green), SC (magenta), and DC (blue).

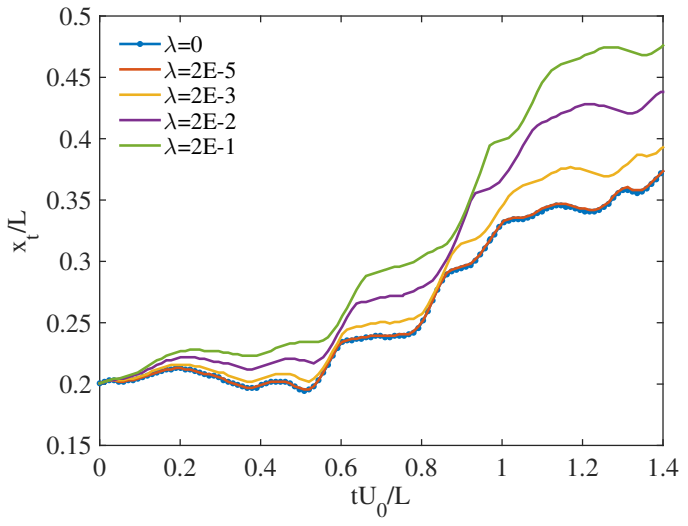

(a) Streamwise location of the flame tip.

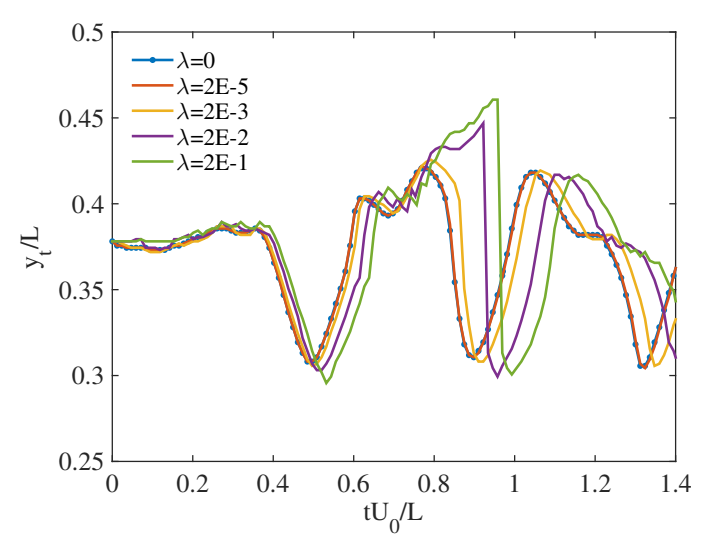

(b) Spanwise location of the flame tip.

Figure 16: Temporal evolution of the (a) streamwise and (b) spanwise locations of the flame tip for different values of $\lambda$. $\lambda=0$ corresponds to the detailed chemistry simulation; $Q_{1}=\left\{Y_{\mathrm{CO}_{2}}, Y_{\mathrm{H}_{2} \mathrm{O}}, Y_{\mathrm{H}_{2}}, Y_{\mathrm{CO}}, Y_{\mathrm{NO}}\right\}$ with model assignment $M_{1}=$ $\{\mathrm{IM}, \mathrm{FPI}, \mathrm{FPV}, \mathrm{SC}\}$. The initial condition is obtained from the steady-state solution with $\lambda=0.2$, which is shown in the third row of Fig. 6.

movement of the flow field in real-time. An example of its dynamic behavior is illustrated in Fig. 18. This case is calculated using $\lambda=0.2$. The shape of each subdomain changes dynamically in response to the flow field, while the topology of the model assignment does not change over time since the unsteady flow field is not strong enough to alter the topology of the flame. The detailed chemistry model consistently occupies the similar proportion of the reacting area and is mainly confined to the fuel-rich side of the flame.

Results at $t U_{0} / L=1$, obtained from the transient PEC-simulation with detailed chemistry simulation (first row) and PEC-simulations with different values for $\lambda$, are shown in Fig. 19. As shown by the scalar fields, the flame is wrinkled by the eddies in the flow but mixing is not significantly enhanced by the unsteady flow field. It can be seen from the temperature field that the reacting area of the flame is relatively unperturbed. The flame tip locations predicted for cases with larger $\lambda$ depart from the reference solution. However, the qualitative behavior of the flame is well described in all cases. The same observation can be made as for the steady cases in Sec. 5.2.1, that the FPI model over-predicts $Y_{\mathrm{CO}}$ by a significant amount. Despite the error in predicting the flame tip location, species mass fractions of $\mathrm{CO}$ and NO for $\lambda=0.2$ clearly show certain unsteady combustion processes that are adequately captured by the detailed chemistry 


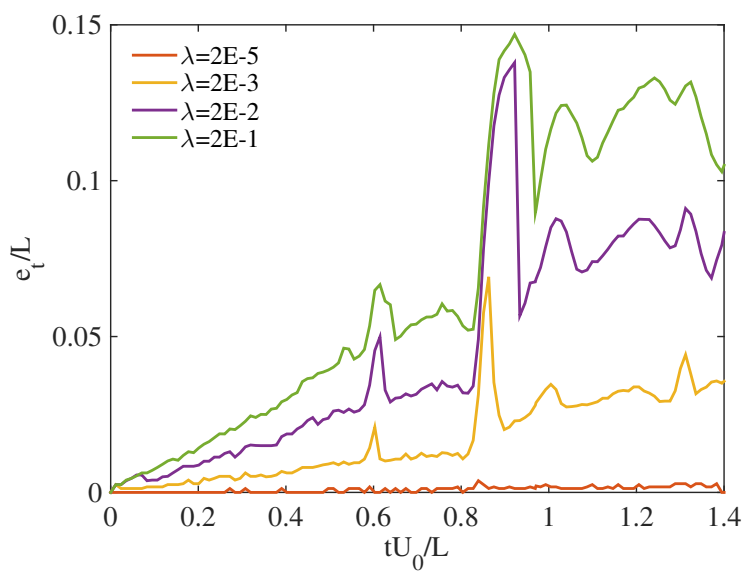

Figure 17: Temporal evolution of the error for the flame tip location for different values of $\lambda$ compared to $\lambda=0$ (the detailed chemistry simulation) .

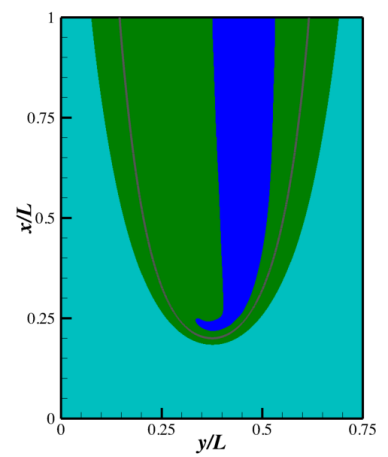

(a) $t U_{0} / L=0$.

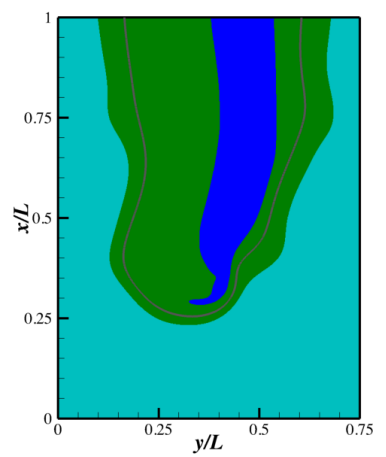

(b) $t U_{0} / L=0.6$.

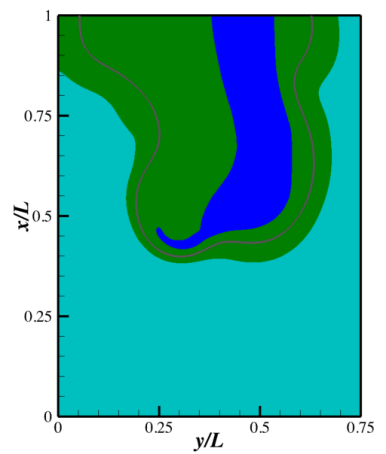

(c) $t U_{0} / L=1.0$.

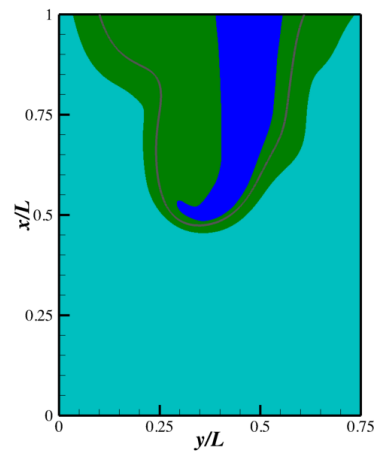

(d) $t U_{0} / L=1.4$.

Figure 18: Model assignment at selected times for the transient PEC-simulation utilizing $M_{1}, Q_{1}$ and $\lambda=0.2$. Model assignment is: IM (cyan), FPI (green), and DC (blue). The grey lines indicate the isoline for $T=400 \mathrm{~K}$.

submodel, thereby improving the accuracy of these quantities. Although the model assignment is dynamically changing in time, all scalar fields remain smooth, which is a consequence of the formulation of the drift term (see discussion in Sec. 3.4.2).

\section{Conclusions}

A Pareto-efficient combustion framework is developed to enable the heterogeneous utilization of different combustion submodels for simulating complex flame configurations. PEC is formulated to require minimal user input, substantially reducing prior expert knowledge about the flame configuration and combustion model selection. User-specific input to the PEC-framework consists of (i) a set of candidate combustion models that can be described by a manifold, (ii) a set of quantities of interest, and (iii) a penalty term to constrain computational cost and desirable model accuracy. Using this information, PEC selects combustion models to describe the chemically reacting flow-field so that the simulation lies on a Pareto front, which is described by optimal accuracy and computational cost of the model assignment. The local model assignment is achieved by evaluating the drift from the manifold of the combustion model. In this work, 


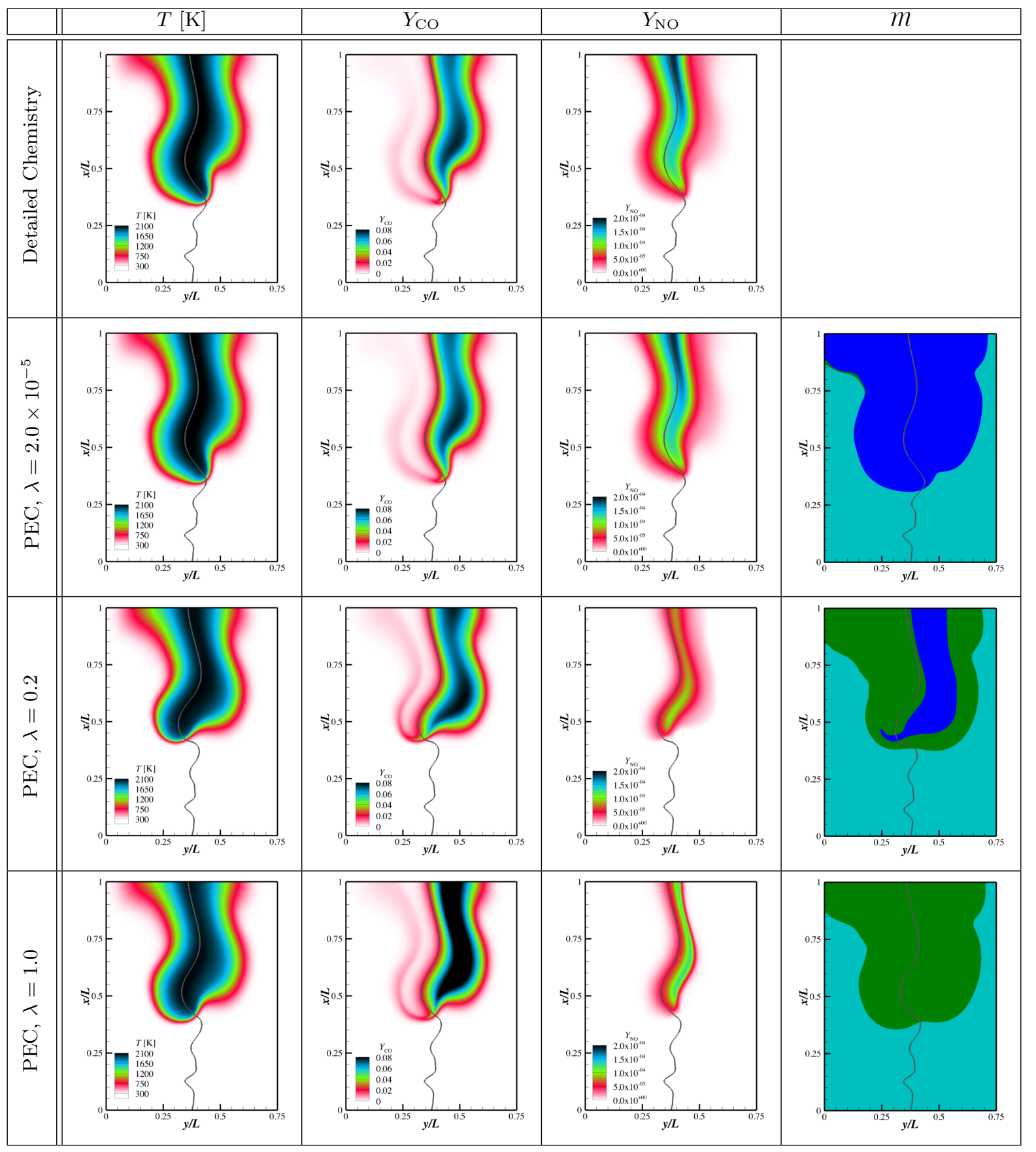

Figure 19: Results from transient PEC-simulations, showing temperature, mass fractions of $\mathrm{CO}$ and NO, and model assignment $m$ at $t U_{0} / L=1$, with $M_{1}=\{\mathrm{IM}, \mathrm{FPI}, \mathrm{FPV}, \mathrm{SC}\}, Q_{1}=\left\{Y_{\mathrm{CO}_{2}}, Y_{\mathrm{H}_{2} \mathrm{O}}, Y_{\mathrm{H}_{2}}, Y_{\mathrm{CO}}, Y_{\mathrm{NO}}\right\}$ for detailed chemistry simulation (first row) and PEC-simulations with $\lambda=2 \times 10^{-5}$ (second row), $\lambda=0.2$ (third row), and $\lambda=1$ (last row); stoichiometric mixture fraction is shown as a gray isoline. Model assignment is: IM (cyan), FPI (green), FPV (red), and DC (blue). 
we consider reaction-transport manifold models (inert mixing, equilibrium, flamelet/progress variable, and flame-prolongation in ILDM) and chemistry manifolds (detailed and skeletal mechanisms), and extensions to other combustion-manifold models can be equally accommodated. Exchange of information across the heterogeneous model assignment is achieved through the formulation of compression and reconstruction operators. The optimal model assignment is obtained as a solution to a point-local minimization problem, which introduces only modest computational overhead.

The capability of the PEC-framework is demonstrated by considering a tribrachial flame configuration and the model performance is examined for different PEC-input parameters. Through direct comparisons of the PEC-modeling results against simulations using detailed chemistry and single-regime combustion models, it was shown that PEC provides an optimal model assignment that is sensible to the quantities of interest, the submodel specification, and the penalty term. While temperature and major species are accurately predicted by flamelet-type reaction-transport models, the description of minor species and pollutants requires the utilization of chemistry-manifold models. Since, however, the utilization of these computationally expensive models is restricted to localized regions of topological and chemical complexity, the computational overhead is manageable and can be directly controlled through the penalty term by the user. The robustness of the PEC-framework is examined in a transient simulation. It is shown that the PEC-adaptation from a singleregime model assignment to the converged PEC-solution is achieved on scales comparable to the convective time-scale, and the model assignment is responsive to the unsteady flow field.

In contrast to the monolithic utilization of a specific combustion model, the PEC-framework provides a general approach for integrating different combustion models to locally adapt the model fidelity. The direct control of the computational cost and accuracy in predicting user-specific target quantities enables the utilization of computationally complex but highly accurate models. By relating the simulation accuracy to the manifold-departure, PEC ensures the utilization of individual models within its intended use without violating underlying model assumptions that are intrinsic to the manifold. As such, this approach overcomes the dependence on combustion regimes and regime-specific combustion models. Furthermore, the general description for integrating different submodels through the manifold drift and the transformation operators allows to accommodate new model developments and model extensions into this Pareto-efficient modeling framework.

\section{Acknowledgments}

The authors gratefully acknowledge financial support from NASA with Award No. NNM13AA11G. This research used resources of the National Energy Research Scientific Computing Center, a DOE Office of Science User Facility supported by the Office of Science of the U.S. Department of Energy under Contract No. DE-AC02-05CH11231.

\section{Appendix A. Nomenclature}

Roman Letters

C Progress variable

$T \quad$ Temperature

$Y_{\alpha} \quad$ Mass fraction of species $\alpha$

$Z \quad$ Mixture fraction

$G^{m} \quad$ Global re-construction operator for model assignment $m$

$H^{m} \quad$ Global compression operator for model assignment $m$

$T^{m^{\prime}, m} \quad$ Global transformation operator from model assignment $m$ to $m^{\prime}$

$M \quad$ Set of candidate combustion models

$Q \quad$ Quantities of interest

$B_{\alpha} \quad$ Scaling factor for the drift of species $\alpha$

$p \quad$ Pressure

$t \quad$ Time 


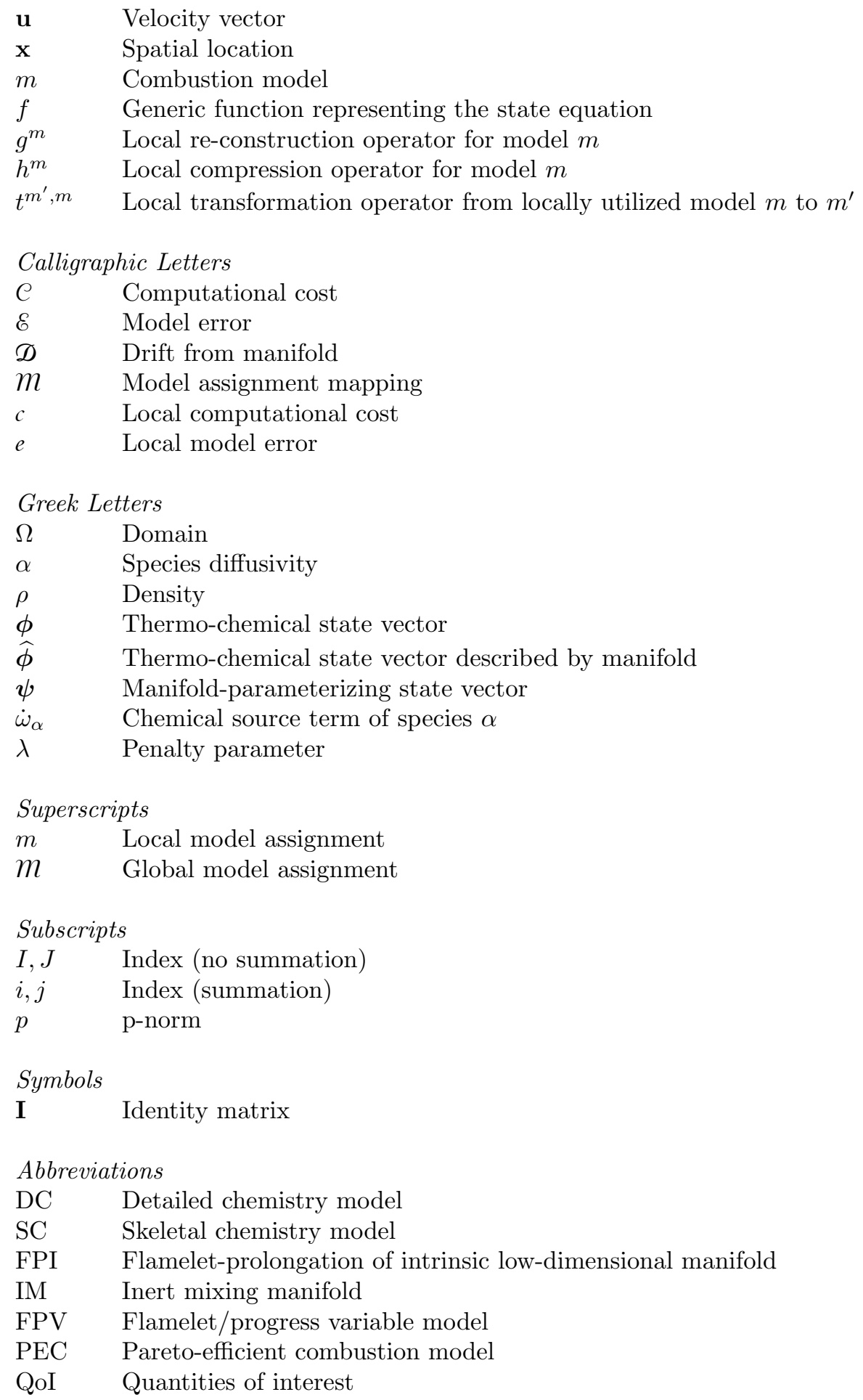

\section{Appendix B. Drift from the reaction-transport manifolds}

The analysis presented in Sec. 3.3.1 is aimed to provide physical insight to the drift from the manifold, D, in Eq. 12 for different reaction-transport manifold models. Transport equations defined in Eqs. 2 and 4, 
as well as the governing equations of the combustion models are transformed to the manifold space spanned by the solution vector $\boldsymbol{\psi}$. Similar analysis has been performed by Pope [1]. Constant transport properties are assumed for simplicity of illustration. Such simplifications are not made for the actual evaluation of $\mathscr{D}$, which follows Eq. 12 directly.

The following relations hold between derivatives in the physical space and in the manifold space,

$$
\begin{aligned}
\nabla \widehat{\phi}_{I} & =\frac{\partial \widehat{\phi}_{I}}{\partial \psi_{i}} \nabla \psi_{i}, \\
\nabla^{2} \widehat{\phi}_{I} & =\frac{\partial^{2} \widehat{\phi}_{I}}{\partial \psi_{i} \partial \psi_{j}} \nabla \psi_{i} \cdot \nabla \psi_{j}+\frac{\partial \widehat{\phi}_{I}}{\partial \psi_{i}} \nabla^{2} \psi_{i} .
\end{aligned}
$$

This allows transforming Eq. 12 as

$$
\mathscr{D}_{I}=\frac{1}{2} \kappa_{i j}^{I} \chi_{i j}+\left(\dot{\omega}_{I}-\frac{\partial \widehat{\phi}_{I}}{\partial \psi_{i}} \dot{\omega}_{i}\right),
$$

where

$$
\kappa_{i j}^{I}=\frac{\partial^{2} \widehat{\phi}_{I}}{\partial \psi_{i} \partial \psi_{j}}
$$

is the manifold curvature with respect to $\{i, j\}$, and

$$
\chi_{i j}=2 \alpha \nabla \psi_{i} \cdot \nabla \psi_{j}
$$

is the corresponding scalar dissipation rate.

Non-premixed flamelet model. The thermodynamic state-space in the FPV-model is obtained from the solution of steady-state flamelet equations. Under the unit Lewis number assumption, these flamelet equations can be written as [26]:

$$
\frac{1}{2} \widehat{\chi}_{Z Z}^{\mathrm{FPV}} \frac{d^{2} \widehat{\boldsymbol{\phi}}^{\mathrm{FPV}}}{d Z^{2}}+\dot{\boldsymbol{\omega}}\left(\widehat{\boldsymbol{\phi}}^{\mathrm{FPV}}\right)=\mathbf{0},
$$

where $\widehat{\phi}^{\mathrm{FPV}}$ combines the vector of species mass fractions and temperature. $d / d Z$ denotes the partial derivative along each individual flamelet for a given scalar dissipation rate. The modeled scalar dissipation rate of mixture fraction $\widehat{\chi}_{Z Z}^{\mathrm{FPV}}$ is an approximation of the actual scalar dissipation rate of mixture fraction $\chi_{Z Z}$. The set of flamelet solutions that is obtained for different values of $\chi_{Z Z \text {,st }}$ is then parameterized in terms of $Z$ and $C$.

Premixed flamelet model. The thermochemical state space for the FPI-model is computed by solving steady unstrained premixed flamelet equations in one dimensional physical coordinate $\xi$ :

$$
u \frac{d}{d \xi}\left(\widehat{\phi}^{\mathrm{FPI}}\right)=\frac{1}{\rho} \frac{d}{d \xi}\left(\rho \alpha \widehat{\phi}^{\mathrm{FPI}}\right)+\dot{\omega}\left(\widehat{\phi}^{\mathrm{FPI}}\right) .
$$

The flamelet equations are solved for different initial mixture conditions, spanning the entire range of flammable mixtures. Similar to the FPV-model, these solutions are tabulated as a function of $\boldsymbol{\psi}^{\mathrm{FPI}}=$ $(Z, C)^{T}$, which shares the same elements as $\psi^{\mathrm{FPV}}$.

Upon transforming Eqs. B.6 and B.7 into the $(Z, C)$-composition space, we can obtain the following uniform expression of $\mathscr{D}$ for both flamelet models:

$$
\mathscr{D}=\frac{1}{2}\left(\chi_{Z Z}-\widehat{\chi}_{Z Z}\right) \kappa_{Z Z}+\left(\chi_{Z C}-\widehat{\chi}_{Z C}\right) \kappa_{Z C}+\frac{1}{2}\left(\chi_{C C}-\widehat{\chi}_{C C}\right) \kappa_{C C} .
$$


For the FPV-model, the modeled scalar dissipation rates are

$$
\begin{aligned}
& \widehat{\chi}_{Z Z}^{\mathrm{FPV}}(Z, Z)=\chi_{Z Z, \mathrm{st}}(Z, C) \frac{\exp \left\{2\left[\operatorname{erfc}^{-1}\left(2 Z_{\mathrm{st}}\right)\right]^{2}\right\}}{\exp \left\{2\left[\operatorname{erfc}^{-1}(2 Z)\right]^{2}\right\}}, \\
& \widehat{\chi}_{C C}^{\mathrm{FPV}}(Z, C)=\widehat{\chi}_{Z Z}^{\mathrm{FPV}}\left(\frac{d C}{d Z}\right)^{2}, \\
& \widehat{\chi}_{Z C}^{\mathrm{FPV}}(Z, C)=\widehat{\chi}_{Z Z}^{\mathrm{FPV}} \frac{d C}{d Z} .
\end{aligned}
$$

For the FPI-model, the modeled scalar dissipation rates are

$$
\begin{aligned}
& \widehat{\chi}_{Z Z}^{\mathrm{FPI}}(Z, C)=0, \\
& \widehat{\chi}_{C C}^{\mathrm{FPI}}(Z, C)=2 \alpha \frac{d^{2} C}{d \xi^{2}}, \\
& \widehat{\chi}_{Z C}^{\mathrm{FPI}}(Z, C)=0 .
\end{aligned}
$$

The expression for $\mathscr{D}$ reveals the physical source of the departure from the non-premixed reactiontransport manifold. The departure is a result of the discrepancy between the modeled scalar dissipation rate and the local scalar dissipation rate in the flow field. The discrepancy is weighted by the manifold curvature, which is large at places where chemical reactions are active. The only distinction between $\mathscr{D}$ for FPV and FPI are the modeled scalar dissipation rates due to the different assumptions on the flame topology in both models. Since the FPI-model uses freely propagating premixed flamelets without stratification or stretch, $\widehat{\chi}_{C C}^{\mathrm{FPI}}$ is the only non-zero contribution since there is no mixture fraction gradient. Note that although $\hat{\chi}_{C C}^{\mathrm{FPV}}$ and $\widehat{\chi}_{Z C}^{\mathrm{FPV}}$ do not appear in Eq. B.6, they are implicitly assumed by the non-premixed flamelet formulation since the variation of species mass fractions is forced to be only along the mixture fraction gradient.

For both FPV- and FPI-models, $\widehat{\chi}$ is pre-determined during the construction of the flamelet table. In the framework of reaction-transport manifolds, it is possible to allow dynamic matching between $\widehat{\chi}$ and $\chi$ as done by Ihme and See [35] and Nguyen et al. [40]. However, the additional flexibility increases the dimensionality of the manifold and would require additional terms in $\boldsymbol{\psi}$ for parameterization. Hence, additional error terms will appear in Eq. B.8. In addition, since the functional relationship between $\widehat{\phi}$ and $\boldsymbol{\psi}$ is sophisticated but constructed indirectly through the modeling of scalar dissipation rates, tabulation is always needed for reaction-transport manifolds, which limits the potential for higher-dimension extensions.

\section{Appendix C. Drift from the skeletal chemical manifold}

Skeletal chemistry models are usually derived from detailed chemistry models, utilizing a smaller number of represented species. The manifold description can also be applied to skeletal chemistry models. Different from a reaction-transport manifold, a skeletal chemistry manifold does not use as reduced set of variables for the manifold solution vector $\boldsymbol{\psi}^{\mathrm{SC}}$, and as a result

$$
\widehat{\phi}^{\mathrm{SC}}=\psi^{\mathrm{SC}} .
$$

The reduction in computational cost comes from a simplified description of the chemical source terms $\widehat{\dot{\boldsymbol{\omega}}}$. The chemical source terms for the represented species are modeled to be independent from the unrepresented quantities, while those for the unrepresented species are assumed to be zero. Hence, when using skeletal chemistry alone the unrepresented species are always zero. Therefore, they are usually not transported explicitly under this scenario despite the fact that they are included in $\boldsymbol{\psi}^{\mathrm{SC}}$. However, when using skeletal chemistry manifolds as a submodel in the PEC-framework, one needs to transport the full set of $\boldsymbol{\psi}^{\mathrm{SC}}$, including the unrepresented species to be compatible with other types of manifolds. Given Eq. C.1, the drift from the manifold for skeletal chemistry models can also be evaluated following the definition in Eq. 12 as

$$
\mathscr{D}=\widehat{\dot{\omega}}_{\phi=\widehat{\phi}}-\dot{\omega}_{\phi=\widehat{\phi}},
$$


which is the error introduced by the simplified chemical source term.

Although the evaluation of $\hat{\dot{\omega}}$ is tractable by design, the full detailed chemistry source terms $\dot{\boldsymbol{\omega}}$ are required to compute the drift from the manifold for skeletal chemistry models, which may introduce significant overhead. There may be workarounds to alleviate the cost of evaluating the drift from the manifold for skeletal chemistry models, but this is beyond the scope of this work.

Equation C.2 also illustrates the distinction between skeletal chemistry manifolds and reaction-transport manifolds. Skeletal chemistry manifolds attempt to approximate chemical source terms while reactiontransport manifolds attempt to model scalar dissipation rates. Since skeletal chemistry manifolds employ no model for the unrepresented species, such manifolds are usually much more expensive to compute than the reaction-transport manifolds due to the higher dimensionality that is required to have a reasonable approximation. However, skeletal chemistry manifolds tend to be more accurate in the presence of complex combustion regimes, since the chemical source terms are less sensitive to the flow field, by which scalar dissipation rates are determined.

\section{Appendix D. Illustration of drift operator}

This appendix illustrates the versatility of the drift term $\mathscr{D}$ for describing relevant ignition and combustion scenarios. For this, we consider IM, FPI, and FPV as manifold submodels to illustrate (i) the detection of autoignition on an inert manifold, (ii) the bootstrap error estimation of the manifold representation, and (iii) the submodel transition at inert/reactive interfaces.

\section{Appendix D.1. Autoignition on an inert manifold}

The first example is relevant for the description of autoignition in the PEC-framework, and considers the transition from an inert mixing manifold to a reactive manifold. For this, we consider a one-dimensional counterflow setup in physical space, with air as oxidizer entering from the left and methane as fuel at the right. Both streams have the same temperature, and the flame is initialized by the unburned mixing solution. The IM-manifold is parameterized by mixture fraction, and is constructed from the linear mixing solution $\widehat{\phi}^{\mathrm{IM}}=\widehat{\phi}_{\mathrm{O}}+Z\left(\widehat{\phi}_{\mathrm{F}}-\widehat{\phi}_{\mathrm{O}}\right)$. The source terms are evaluated from the scalars on the IM-manifold as $\dot{\omega}^{\mathrm{IM}}=\dot{\omega}\left(\widehat{\phi}^{\mathrm{IM}}\right)$. Since these source terms are local to the manifold they can be precomputed, so that the entire manifold representation is tabulated prior to the simulation.

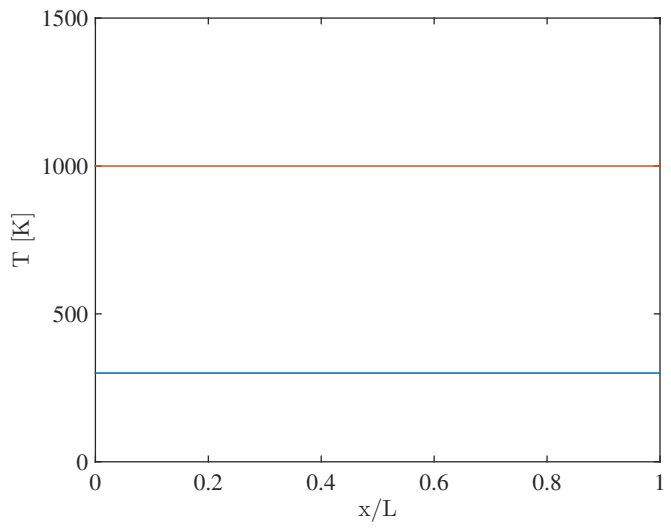

(a) Temperature.

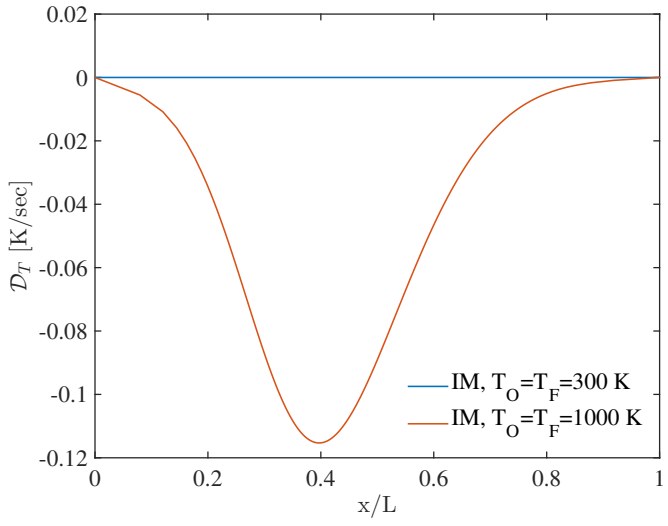

(b) Drift term.

Figure D.20: Autoignition of an unburned mixture in a counterflow flame with air as oxidizer entering from the left and methane on the right; (a) initial temperature profile and (b) drift from IM-manifold with $Q=T$.

In this example we consider the temperature as quantity of interest, $Q=T$, and Eq. (12) reduces to $\mathscr{D}_{T}^{\mathrm{IM}}=\left.\dot{\omega}_{T}\right|_{T=\widehat{T}^{\mathrm{IM}}}$, since $\partial_{Z^{\mathrm{IM}}} \widehat{T}^{\mathrm{IM}}=0$ and $\left.\nabla T\right|_{T=\widehat{T}^{\mathrm{IM}}}=0$. The drift is evaluated for two cases with 
$T_{\mathrm{O}}=T_{\mathrm{F}}=300 \mathrm{~K}$ (inert mixture below the cross-over temperature) and $T_{\mathrm{O}}=T_{\mathrm{F}}=1000 \mathrm{~K}$ (autoigniting mixture above cross-over condition); see Fig. 20(a). The drift term for temperature is evaluated from the tabulation and illustrated in Fig. 20(b). Since the inert mixing solution is a realizable solution for the lowtemperature condition, $\mathscr{D}_{T}^{\mathrm{IM}}=0$, confirming that this flame state is adequately described by the IM-model. However, since the high-temperature condition is prone to autoignition, $\mathscr{D}_{T}^{\mathrm{IM}}=\left.\dot{\omega}_{T}\right|_{\widehat{\phi}=\widehat{\phi}^{\mathrm{IM}}} \neq 0$, indicating that the solution departs from the IM-manifold, and a transition to a reaction-transport manifold model is required to describe the ignition process.

\section{Appendix D.2. Bootstrap error estimation of manifold}

This example demonstrates the capability of using the drift term as bootstrap error estimate of a particular manifold representation. For this, we consider the temporal evolution of a one-dimensional counterflow diffusion flame. The initial condition corresponds to a burning flamelet solution near the quenching condition, and the temporal evolution is obtained from the transient solution of the FPV and the FPI models, which are described by transport equations for the mixture fraction and progress variable. The stoichiometric scalar dissipation rate is $1 \mathrm{sec}^{-1}$. Thermochemical transport properties are taken from the respective diffusion and premixed reaction-transport manifolds. The solutions of the reaction progress variable and the CO-mass fraction are shown in Fig. 21(a). We select the CO-mass fraction as quantity of interest.

Although both results are obtained as self-consistent solutions to the different combustion models, the drift term can be used as bootstrap error estimation of each manifold model. This is illustrated in Fig. 21(b), in which the drift term for the CO-mass fraction is evaluated for both models. Consistent with the underlying flame structure, the drift term for the FPV-model is zero, confirming that the non-premixed flamelet model provides an adequate description of this flame. In contrast, the non-zero value for $\mathscr{D}_{\mathrm{CO}}^{\mathrm{FPI}}$ indicates that the solution obtained from the FPI premixed reaction-transport manifold is inadequate in representing this particular flame structure within the reaction zone. While these results are not unexpected, this analysis

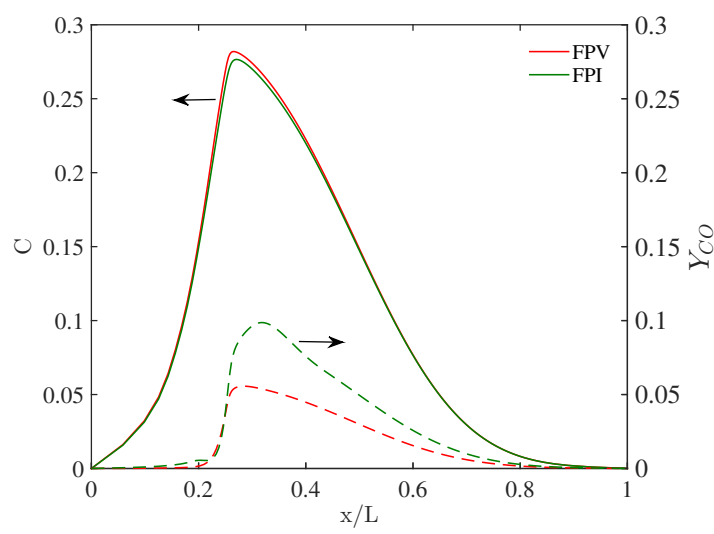

(a) Progress variable.

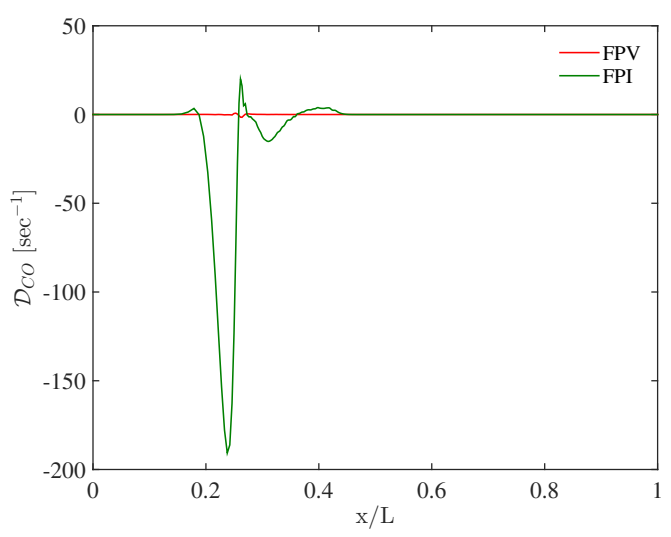

(b) Drift term $\mathscr{D}_{\mathrm{CO}}$.

Figure D.21: Illustration of using drift function as bootstrap error estimation of the manifold for one-dimensional counterflow diffusion flame; (a) progress variable obtained from FPV and FPI solution, (b) CO mass fraction as quantity of interest, and (c) drift term $\mathscr{D}_{\mathrm{CO}}$ evaluated from the FPV and FPI submodels.

emphasizes that the drift term can be used a self-contained error estimate for a particular flame solution, independent of external information or a reference solution that would otherwise be need as measure of the departure.

\section{Appendix D.3. Submodel transition at interface}

The last example examines the transition from an IM-manifold submodel to a reaction-transport manifold model. For this, we consider the temporal evolution of a stoichiometric premixed methane/air flame that 
evolves in physical space. The PEC-submodel assignment consists of IM and FPI combustion models. The spatio-temporal evolution of the flame is described from the solution of a transport equation for the reaction progress variable, and thermo-transport properties and the source term for the progress variable are taken from the respective chemistry libraries. The quantity of interest is $Q=Y_{\mathrm{CO}}, \lambda=0.1$, and the cost assignments are $c_{\mathrm{IM}}=0.01$ and $c_{\mathrm{FPI}}=0.02$ (see Table. 1$)$.

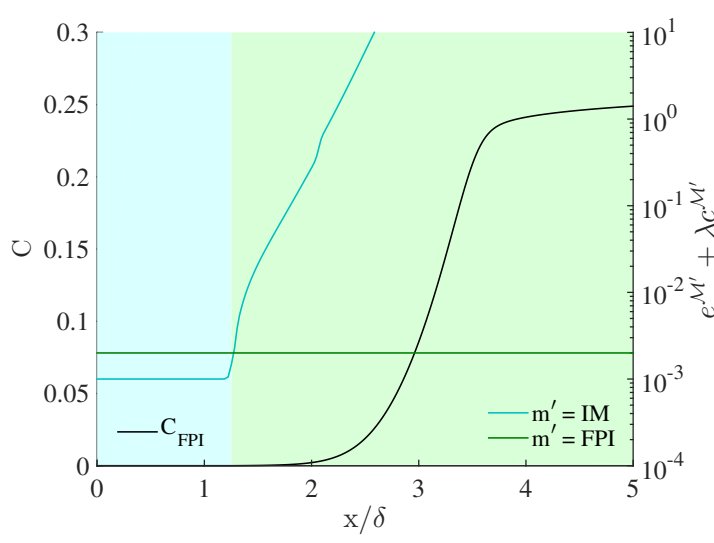

(a) One-dimensional premixed methane/air flame,

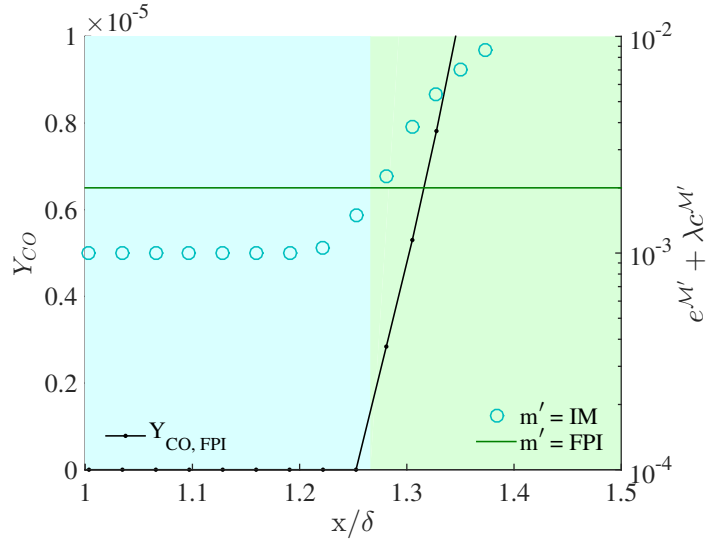

(b) Zoom-in at the interface.

Figure D.22: Evaluation of PEC-cost function for one-dimensional freely propagating stoichiometric methane/air flame, showing the spatial evolution of the progress variable, the cost-function and the corresponding submodel assignment. $\delta$ is the flamethickness. PEC-parameters: $Q=Y_{\mathrm{CO}}, \lambda=0.1, M=\{\mathrm{IM}, \mathrm{FPI}\}$.

Results from the PEC-simulation are presented in Fig. D.22, showing the spatial evaluation of the progress variable (black) and the results from the cost-function evaluation and model assignment. To obtain the submodel assignment, we evaluate the cost function $e^{m^{\prime}}(y)+\lambda c^{m^{\prime}}$ which is computed using the drift term (Eq. (12)) and assigned computational cost for each model. Following the procedure described in Algorithm $1, m^{\prime}$ is obtained by locally replacing $m$ from the previous time step with the candidate models $m^{\prime} \in M$. The cost functions for both models are shown in Fig. D.22, from which the corresponding submodel assignments follow directly: In the region $x / \delta<1.25$, contributions to the cost function are only from the computational cost of each model, and both submodels accurately capture the inert region upstream of the preheat zone. Since $c_{\mathrm{IM}}<c_{\mathrm{FPI}}$, the IM-submodel is assigned to this region. Entering the preheat zone, the error in representing the flame-structure by the inert mixing manifold increases substantially and exceeds that of the FPI-model. Therefore, the submodel assignment switches from IM to FPI. As the flame propagates through the domain, this submodel assignment will move with the flame. It is noted that more sensitive scalars can be incorporated into the definition of $Q$ to detect preheat zones and autoignition kernels.

\section{References}

[1] S. B. Pope. Small scales, many species and the manifold challenges of turbulent combustion. Proc. Combust. Inst., 34:1-31, 2012.

[2] U. Maas and S. B. Pope. Simplifying chemical kinetics: Intrinsic low-dimensional manifolds in composition space. Combust. Flame, 88(3-4):239-264, 1992.

[3] S. H. Lam and D. A. Goussis. The CSP method for simplifying kinetics. Int. J. Chem. Kinet., 26:461-486, 1994.

[4] T. Lu and C. K. Law. A directed relation graph method for mechanism reduction. Proc. Combust. Inst., 30:1333-1341, 2005.

[5] P. Pepiot-Desjardins and H. Pitsch. An efficient error-propagation-based reduction method for large chemical kinetic mechanisms. Combust. Flame, 154:67-81, 2008.

[6] J. A. van Oijen, F. A. Lammers, and L. P. H. de Goey. Modeling of complex premixed burner systems by using flameletgenerated manifolds. Combust. Flame, 127(3):2124-2134, 2001. 
[7] O. Gicquel, N. Darabiha, and D. Thevenin. Laminar premixed hydrogen/air counterflow flame simulations using flame prolongation of ILDM with differential diffusion. Proc. Combust. Inst., 28:1901-1908, 2000.

[8] V. Bykov and U. Maas. The extension of the ILDM concept to reaction-diffusion manifolds. Combust. Theor. Model., 11(6):839-862, December 2007.

[9] C. D. Pierce and P. Moin. Progress-variable approach for large-eddy simulation of non-premixed turbulent combustion. J. Fluid Mech., 504:73-97, 2004.

[10] J. C. Keck and D. Gillespi. Rate-controlled partial-equilibrium method for treating reacting gas-mixtures. Combust. Flame, 17(2):237-241, 1971.

[11] Z. Ren, G. M. Goldin, V. Hiremath, and S. B. Pope. Reduced description of reactive flows with tabulation of chemistry. Combust. Theor. Model., 15(6):827-848, 2011.

[12] V. Hiremath, Z. Ren, and S. B. Pope. A greedy algorithm for species selection in dimension reduction of combustion chemistry. Combust. Theor. Model., 14(5):619-652, 2010.

[13] J. C. Sutherland and A. Parente. Combustion modeling using principal component analysis. Proc. Combust. Inst., 32(1):1563-1570, 2009

[14] A. Coussement, O. Gicquel, and A. Parente. Kernel density weighted principal component analysis of combustion processes. Combust. Flame, 159(9):2844-2855, 2012.

[15] Y. Yang, S. B. Pope, and J. H. Chen. Empirical low-dimensional manifolds in composition space. Combust. Flame, 160(10):1967-1980, 2013.

[16] H. Mirgolbabaei and T. Echekki. Nonlinear reduction of combustion composition space with kernel principal component analysis. Combust. Flame, 161(1):118-126, 2014.

[17] F. A. Williams. Progress in knowledge of flamelet structure and extinction. Prog. Energy Combust. Sci., 26(4-6):657-682, 2000.

[18] N. Peters. Turbulent Combustion. Cambridge University Press, Cambridge, 2000.

[19] T. Poinsot and D. Veynante. Theoretical and Numerical Combustion. R.T. Edwards, Inc., Philadelphia, PA, 2001.

[20] D. Veynante and L. Vervisch. Turbulent combustion modeling. Prog. Energy Combust. Sci., 28(3):193-266, 2002.

[21] H. Pitsch. Large-eddy simulation of turbulent combustion. Annu. Rev. Fluid Mech., 38:453-482, 2006.

[22] B. F. Magnussen and B. H. Hjertager. On mathematical modeling of turbulent combustion with special emphasis on soot formation and combustion. Proc. Combust. Inst., 16(1):719-729, 1977.

[23] S. B. Pope. PDF methods for turbulent reactive flows. Prog. Energy Combust. Sci., 11(2):119-192, 1985.

[24] D. C. Haworth. Progress in probability density function methods for turbulent reacting flows. Prog. Energy Combust. Sci., 36(2):168-259, 2010.

[25] S. P. Burke and T. E. W. Schumann. Diffusion flames. Ind. Eng. Chem., 20(10):998-1004, 1928.

[26] N. Peters. Laminar diffusion flamelet models in non-premixed turbulent combustion. Prog. Energy Combust. Sci., 10(3):319-339, 1984

[27] J. A. van Oijen and L. P. H. de Goey. Modelling of premixed laminar flames using flamelet-generated manifolds. Combust. Sci. Tech., 161:113-137, 2000.

[28] M. Ihme, C. M. Cha, and H. Pitsch. Prediction of local extinction and re-ignition effects in non-premixed turbulent combustion using a flamelet/progress variable approach. Proc. Combust. Inst., 30:793-800, 2005.

[29] B. Fiorina, R. Baron, O. Gicquel, D. Thevenin, S. Carpentier, and N. Darabiha. Modelling non-adiabatic partially premixed flames using flame-prolongation of ILDM. Combust. Theor. Model., 7:449-470, 2003.

[30] L. Shunn. Large-Eddy Simulation of Combustion Systems with Convective Heat-Loss. PhD thesis, Stanford University, 2009.

[31] D. J. Lee, S. Thakur, J. Wright, M. Ihme, and W. Shyy. Characterization of flow field structure and species composition in a shear coaxial rocket GH2/GO2 injector: Modeling of wall heat losses. AIAA Paper 2011-6125, 2011.

[32] M. Ihme and H. Pitsch. Modeling of radiation and NO formation in turbulent non-premixed flames using a flamelet/progress variable formulation. Phys. Fluids, 20:055110, 2008.

[33] P. Domingo, L. Vervisch, and D. Veynante. Large-eddy simulation of a lifted methane jet flame in a vitiated coflow. Combust. Flame, 152:415-432, 2008.

[34] J.-B. Michel, O. Colin, C. Angelberger, and D. Veynante. Using the tabulated diffusion flamelet model ADF-PCM to simulate a lifted methane-air jet flame. Combust. Flame, 156:1318-1331, 2009.

[35] M. Ihme and Y. C. See. Prediction of autoignition in a lifted methane/air flame using an unsteady flamelet/progress variable model. Combust. Flame, 157:1850-1862, 2010.

[36] C. Hasse and N. Peters. A two mixture fraction flamelet model applied to split injection in a DI Diesel engine. Proc. Combust. Inst., 30:2755-2762, 2005.

[37] D. J. Cook, H. Pitsch, J. H. Chen, and E. R. Hawkes. Flamelet-based modeling of auto-ignition with thermal inhomogeneities for application to HCCI engines. Proc. Combust. Inst., 31(2):2903-2911, 2007.

[38] M. Ihme and Y. C. See. LES flamelet modeling of a three-stream MILD combustor: Analysis of flame sensitivity to scalar inflow conditions. Proc. Combust. Inst., 33:1309-1317, 2010.

[39] E. Knudsen and H. Pitsch. Capabilities and limitations of multi-regime flamelet combustion models. Combust. Flame, $159: 242-264,2012$.

[40] P.-D. Nguyen, V. Vervisch, V. Subramanian, and P. Domingo. Multidimensional flamelet-generated manifolds for partially premixed combustion. Combust. Flame, 157:43-61, 2010.

[41] A. S. Almgren, J. B. Bell, P. Colella, L. H. Howell, and M. L. Welcome. A conservative adaptive projection method for the variable density incompressible navier-stokes equations. J. Comp. Phys., 142(1):1 - 46, 1998.

[42] W. E, B. Engquist, and Y. Sun. The heterogeneous multiscale methods with application to combustion. In Turb. Combust. 
Model., chapter 18. Springer, 2011.

[43] W. Stadler. A survey of multicriteria optimization or the vector maximum problem, part I. J. Optim. Theory Appl., 29(1):1-52, 1979

[44] Y. Boykov, O. Veksler, and R. Zabih. Fast approximate energy minimization via graph cuts. IEEE Trans. Pattern Anal. Mach. Intell., 23(11):1222-1239, 2001.

[45] Y. A. Lecun, L. Bottou, G. B. Orr, and K.-R. Müller. Efficient BackProp. In G. Montavon, G. B. Orr, and K.-R. Müller, editors, Neural networks: Tricks of the trade, volume 7700 of Lecture Notes in Computer Science, pages 9-48. Springer, 2012.

[46] C. Taylor. Classification and kernel density estimation. Vistas Astron., 41(3):411-417, 1997.

[47] T. Hastie, R. Tibshirani, and J. Friedman. The Elements of Statistical Learning. Springer Series in Statistics. Springer New York, New York, NY, 2009.

[48] T. Echekki and J. Chen. Structure and propagation of methanol-air triple flames. Combust. Flame, 114:231-245, 1998.

[49] J. Oijen and L. De Goey. A numerical study of confined triple flames using a flamelet-generated manifold. Comb. Theor. Model., 8:141-163, 2006.

[50] S. H. Chung. Stabilization, propagation and instability of tribrachial triple flames. Proc. Combust. Inst., 31:877-892, 2007.

[51] G. R. Ruetsch, L. Vervisch, and A. Liñán. Effects of heat release on triple flame. Phys. Fluids, 7:1447-1454, 1995.

[52] M. Herrmann, P. Terhoeven, B. Binninger, and N. Peters. Numerical simulation of partially premixed combustion using the flamelet approach. J. Appl. Math. Mech., 79:41-43, 1999.

[53] G. P. Smith, D. M. Golden, M. Frenklach, N. W. Moriarty, B. Eiteneer, M. Goldenberg, C. T. Bowman, R. K. Hanson, S. Song, W. C. Gardiner, Jr., V. V. Lissianski, and Z. Qin. GRI-Mech 3.0, 2000. available from http://www. me. berkeley. edu/gri-mech/.

[54] H. Pitsch. FlameMaster v3.1: A C++ computer program for 0D combustion and 1D laminar flame calculations, 1998.

[55] J. Bell, M. Day, J. Grcar, and M. Lijewski. Active control for statistically stationary turbulent premixed flame simulations. Comm. Appl. Math. Comp. Sci., 1(1):29-51, 2006.

[56] A. Kazakov and M. Frenklach. DRM19, 1994. available from http://www.me.berkeley.edu/drm/.

[57] M. Chen, M. Herrmann, and N. Peters. Flamelet moldeling of lifted turbulent methane/air and propane/air jet diffusion flames. Proc. Combust. Inst., 28:169-174, 2000.

[58] M. Ihme, L. Shunn, and J. Zhang. Regularization of reaction progress variable for application to flamelet-based combustion models. J. Comp. Phys., 231:7715-7721, 2012.

[59] H. Yamashita, M. Shimada, and T. Takeno. A numerical study on flame stability at the transition point of jet diffusion flames. Proc. Combust. Inst., 26:27-34, 1996. 

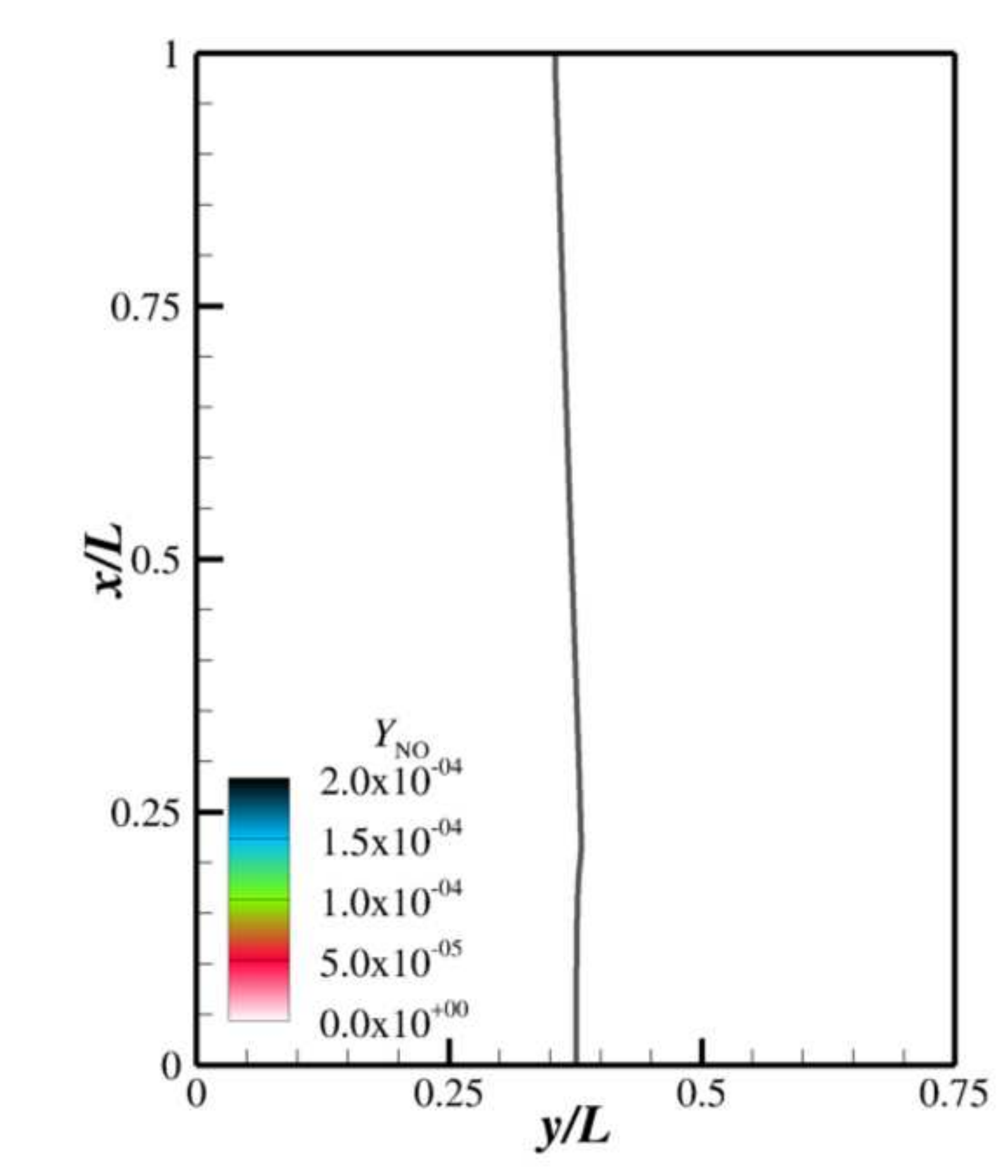

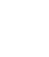




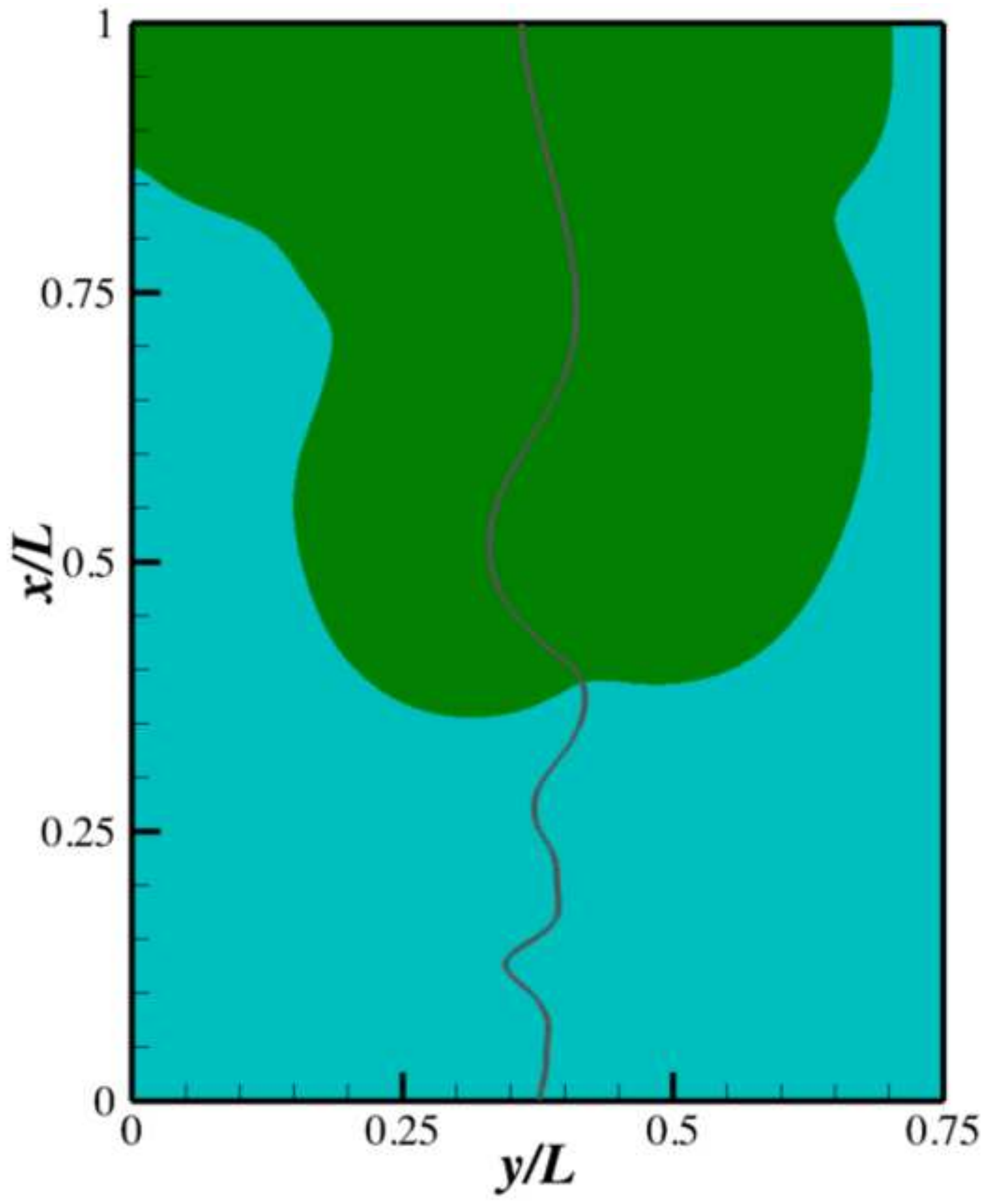




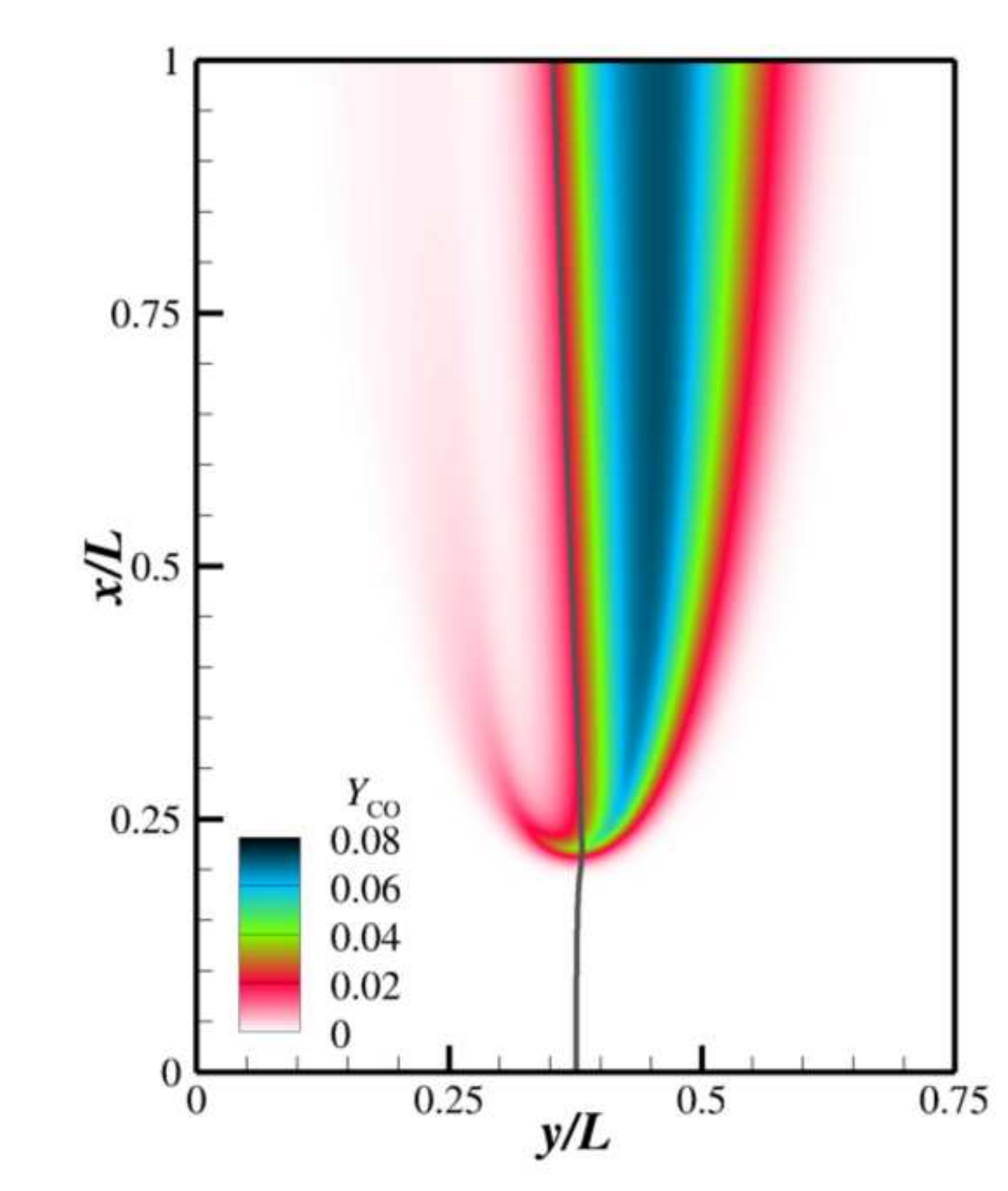




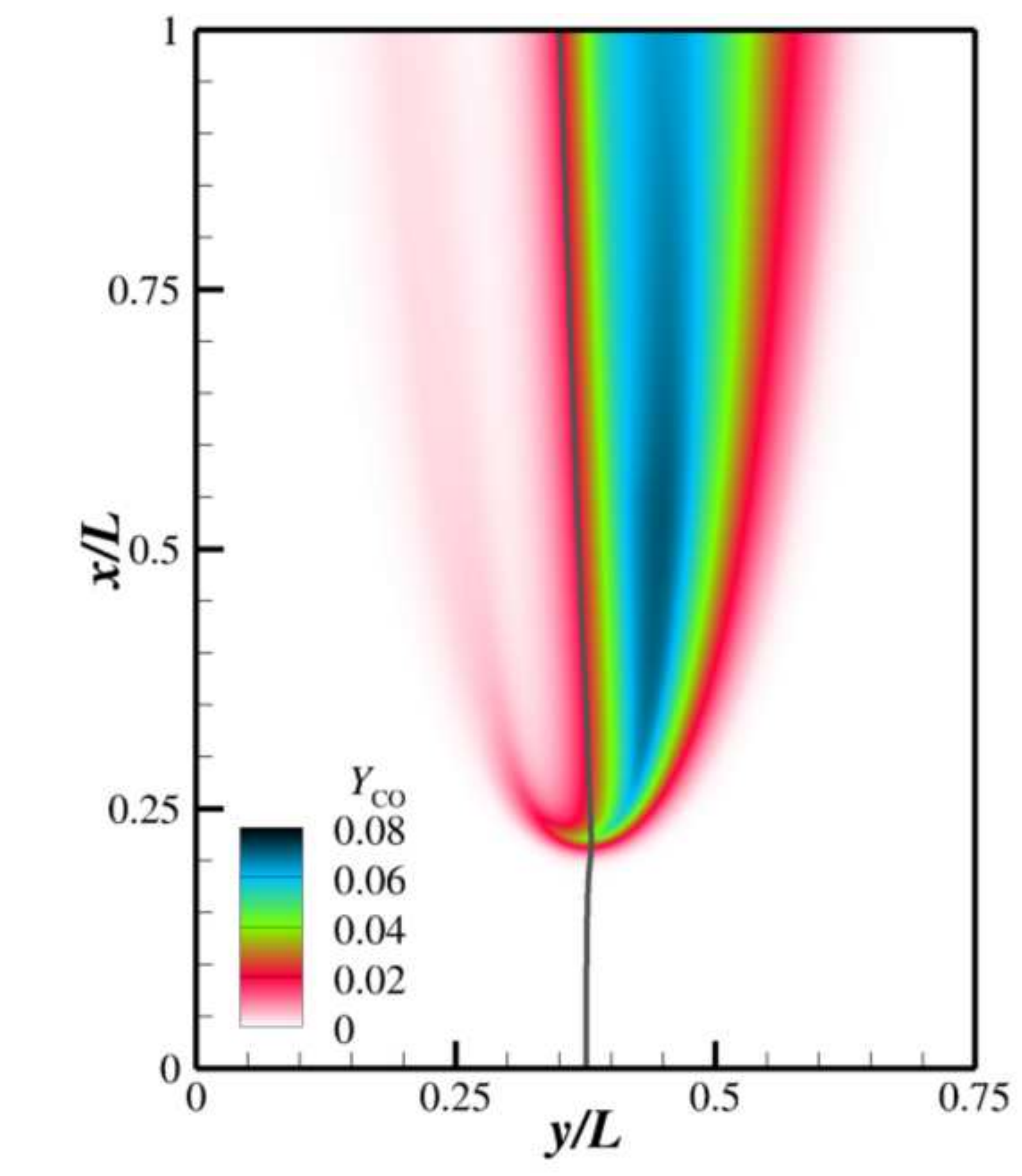




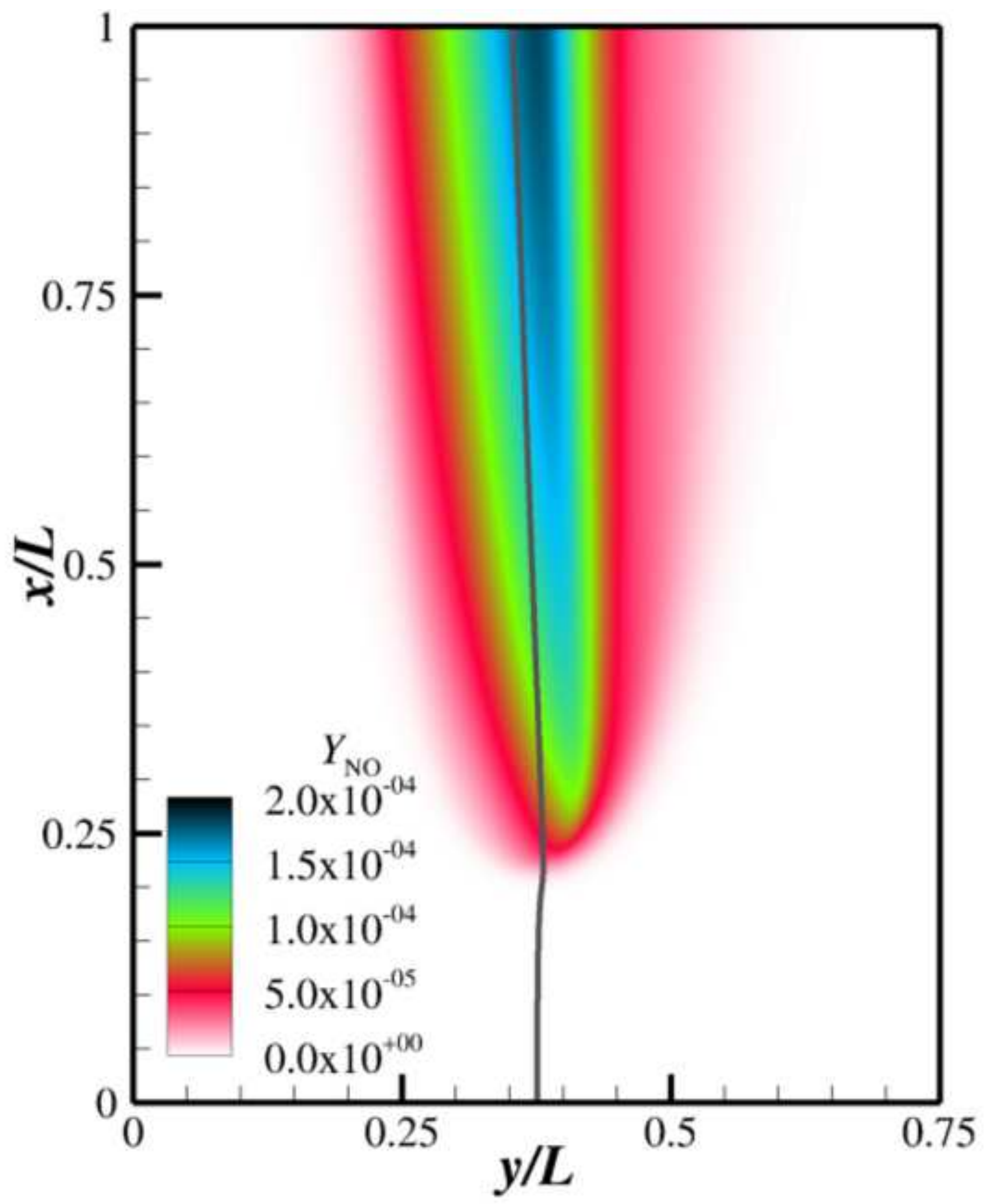




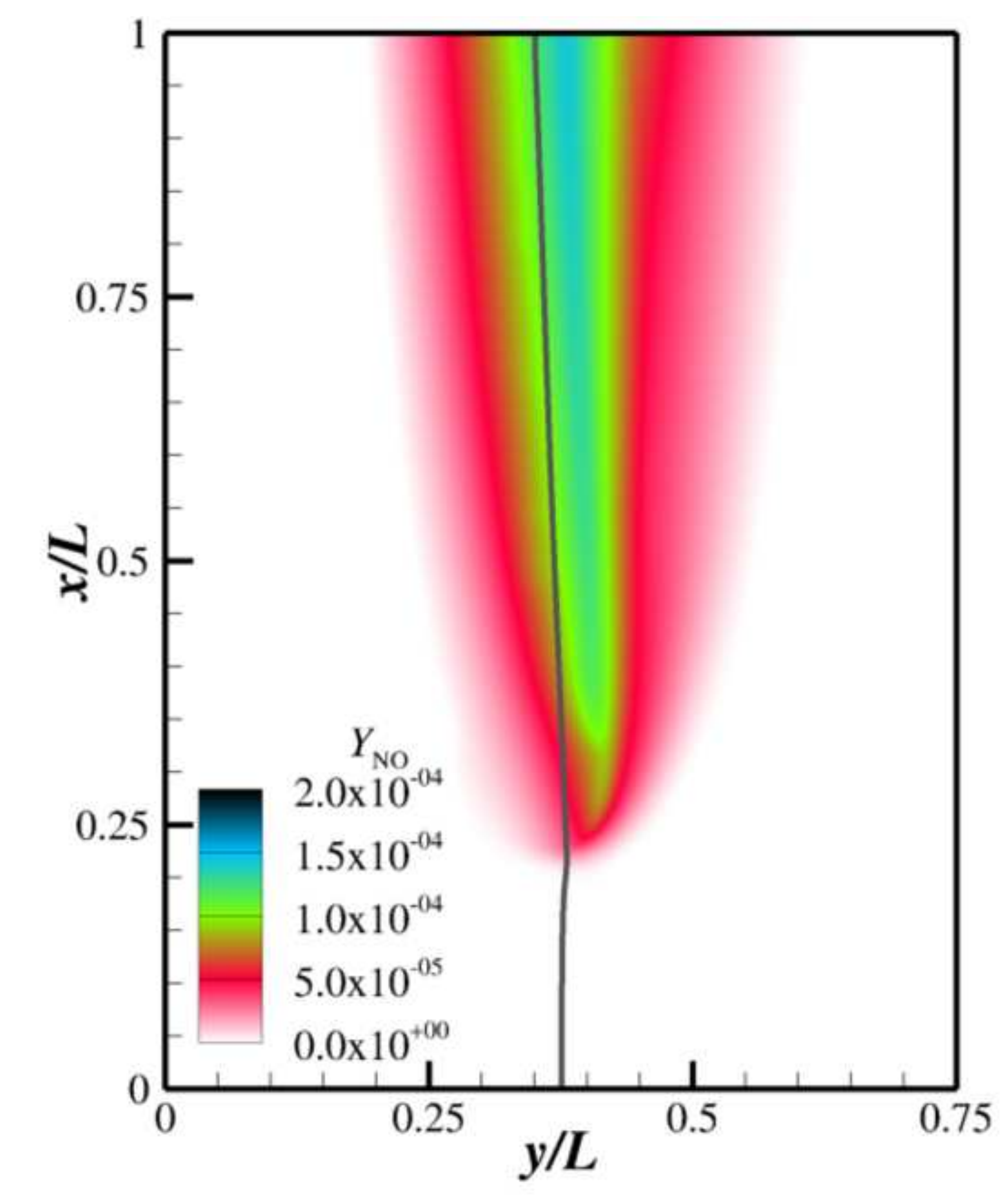



fam_big_t_m1_lamb_0p002.png

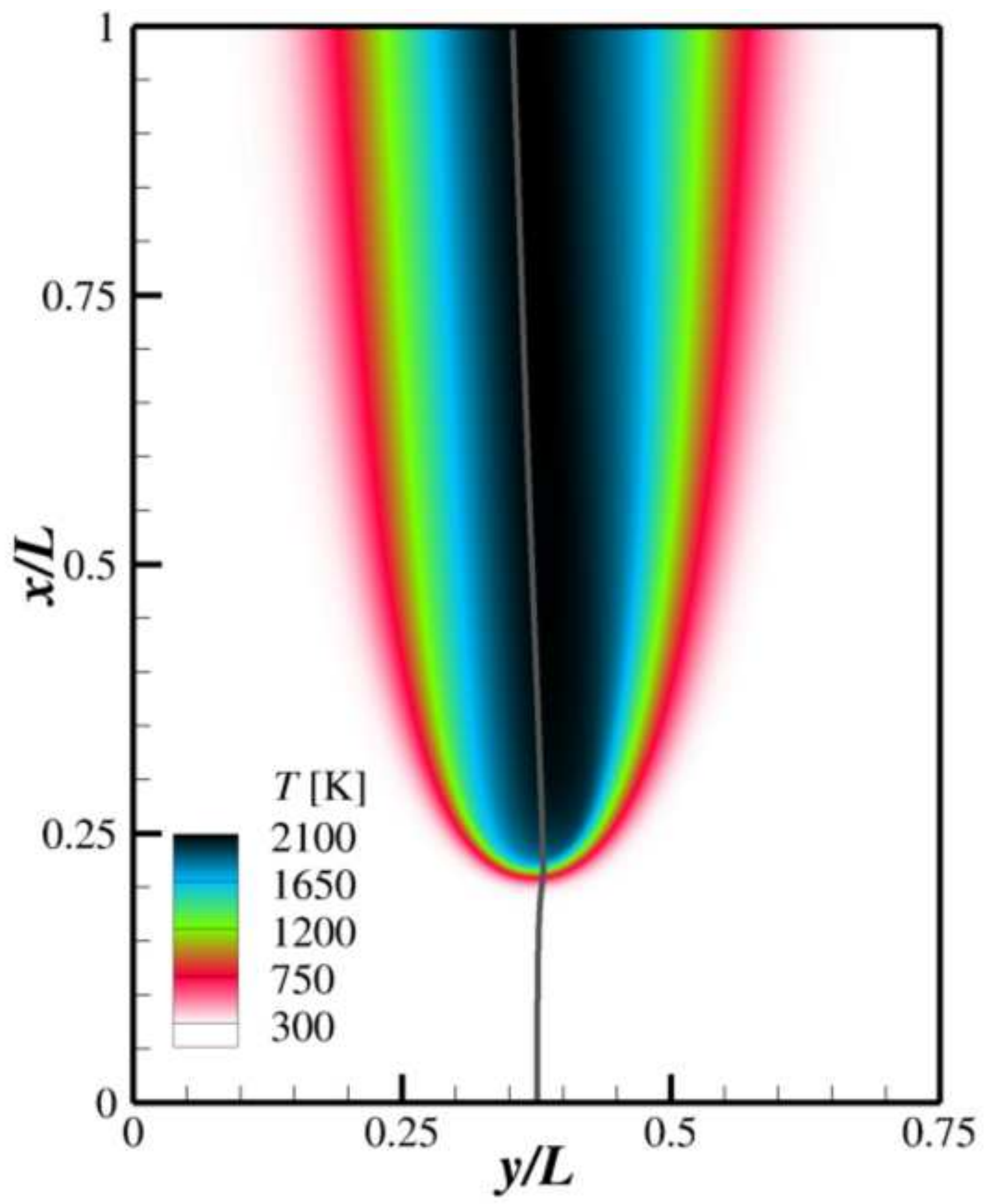



fam_big_t_m2_lamb_0p100.png

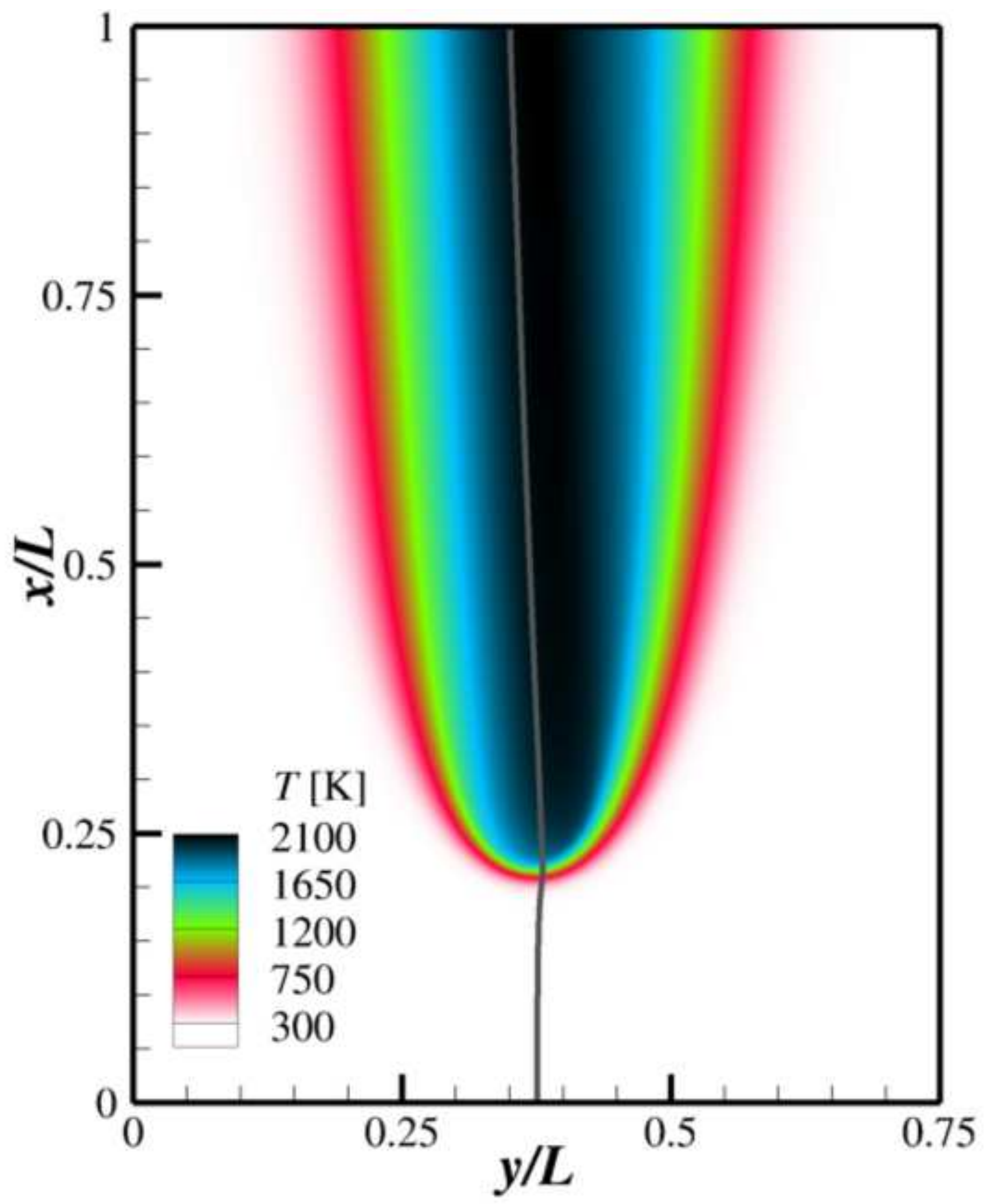



fam_big_z_m1_lamb_0p002.png

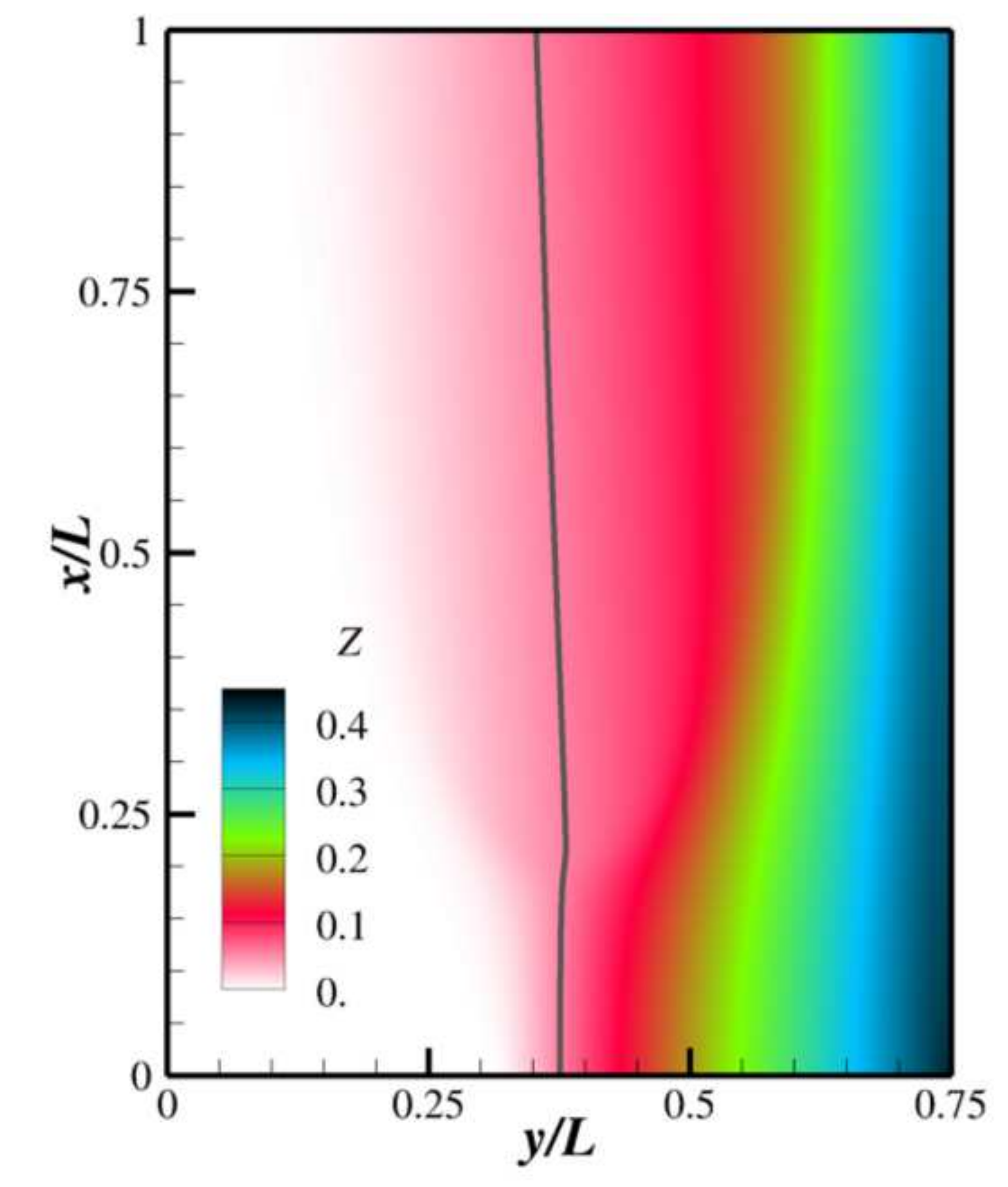

(

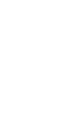

.

(2)



fam_big_z_m1_lamb_0p020.png

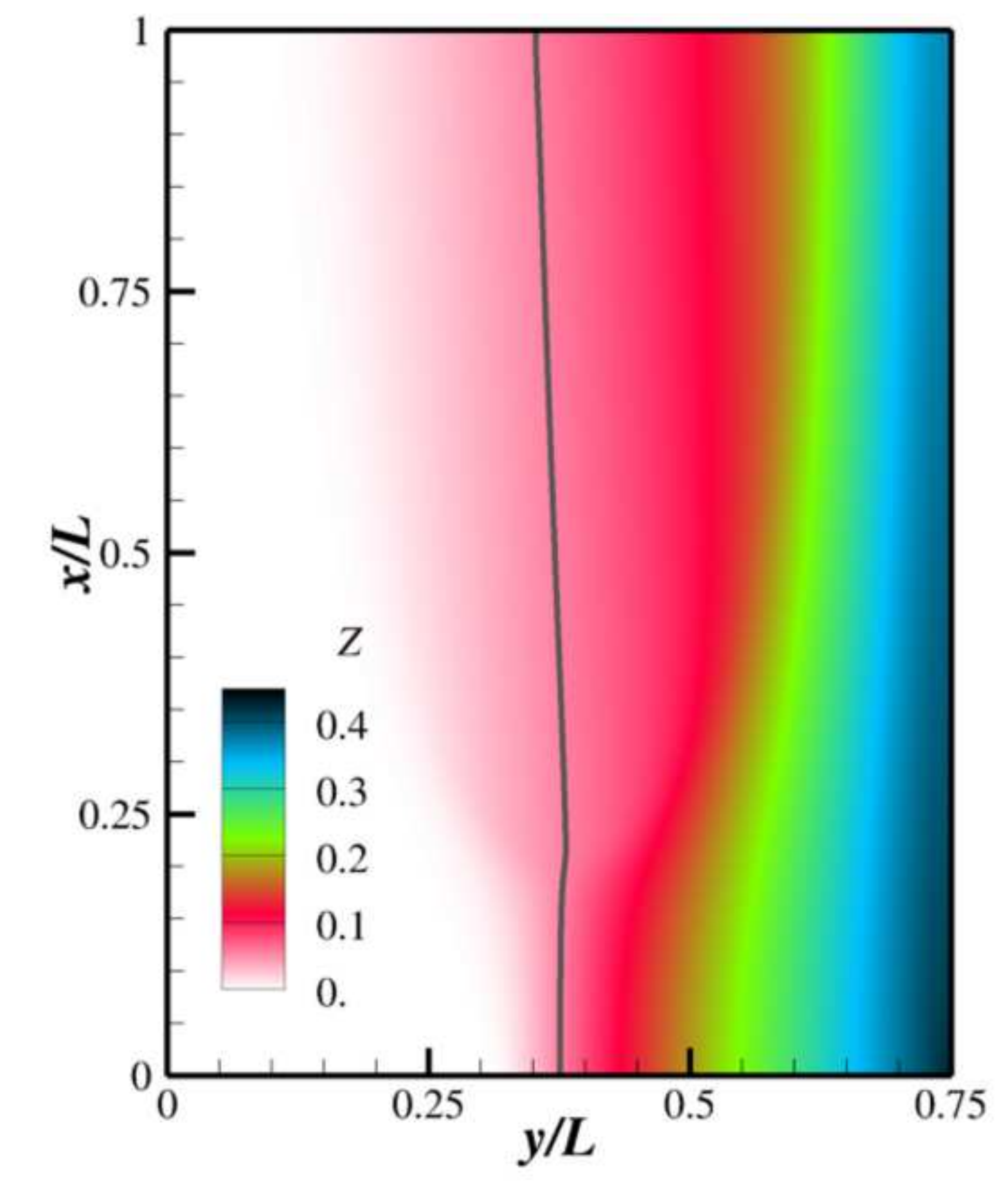

(⿸丆口

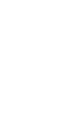

. 

fam_big_z_m1_lamb_0p200.png

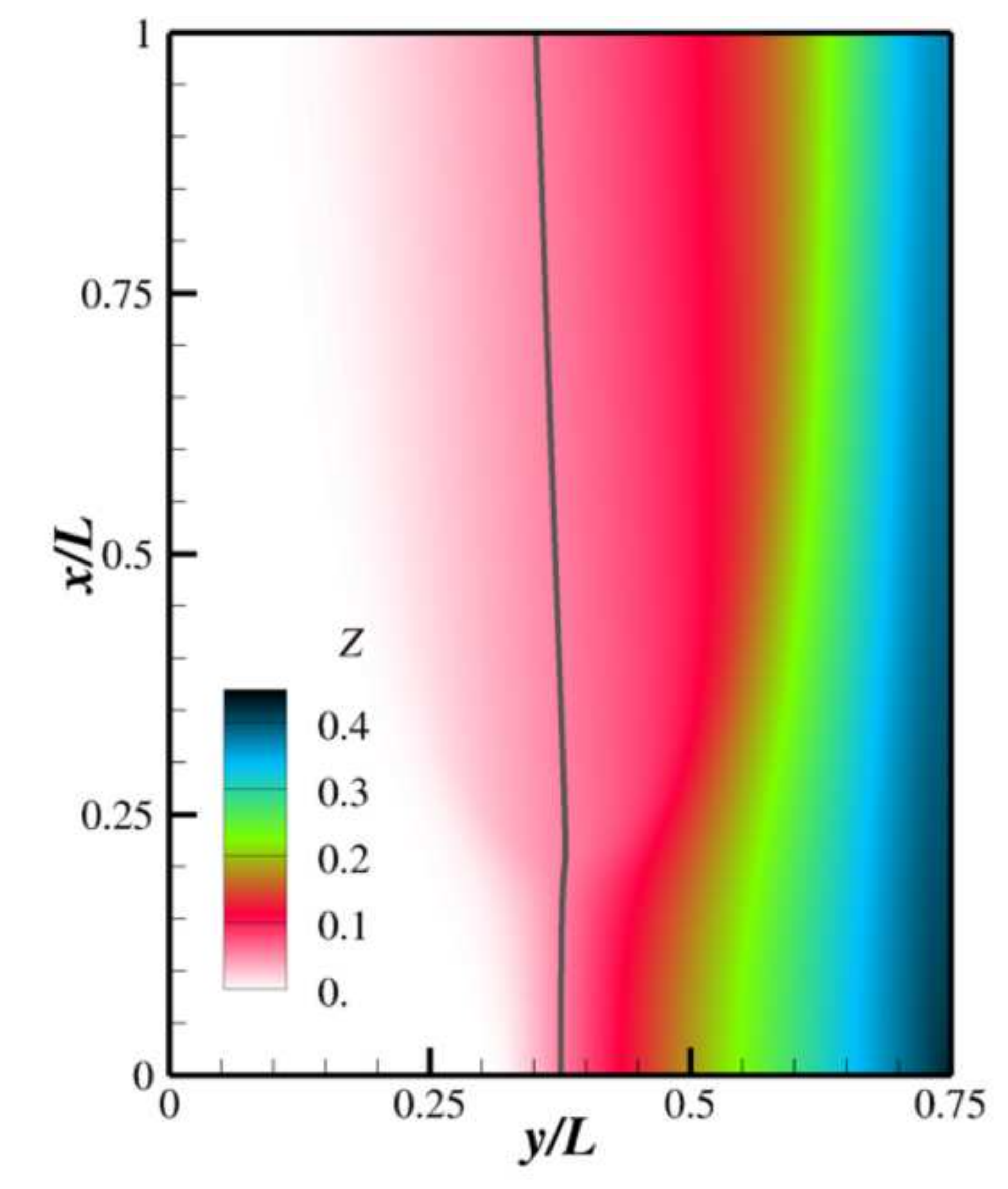

(⿸丆口

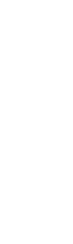

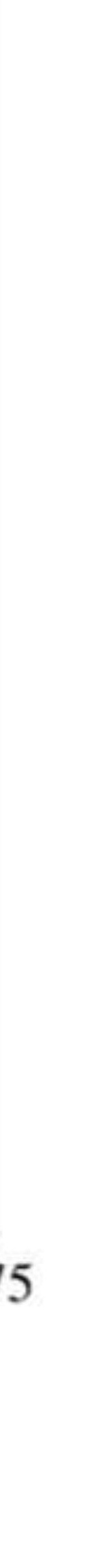



fam_big_z_m1_lamb_2p000.png

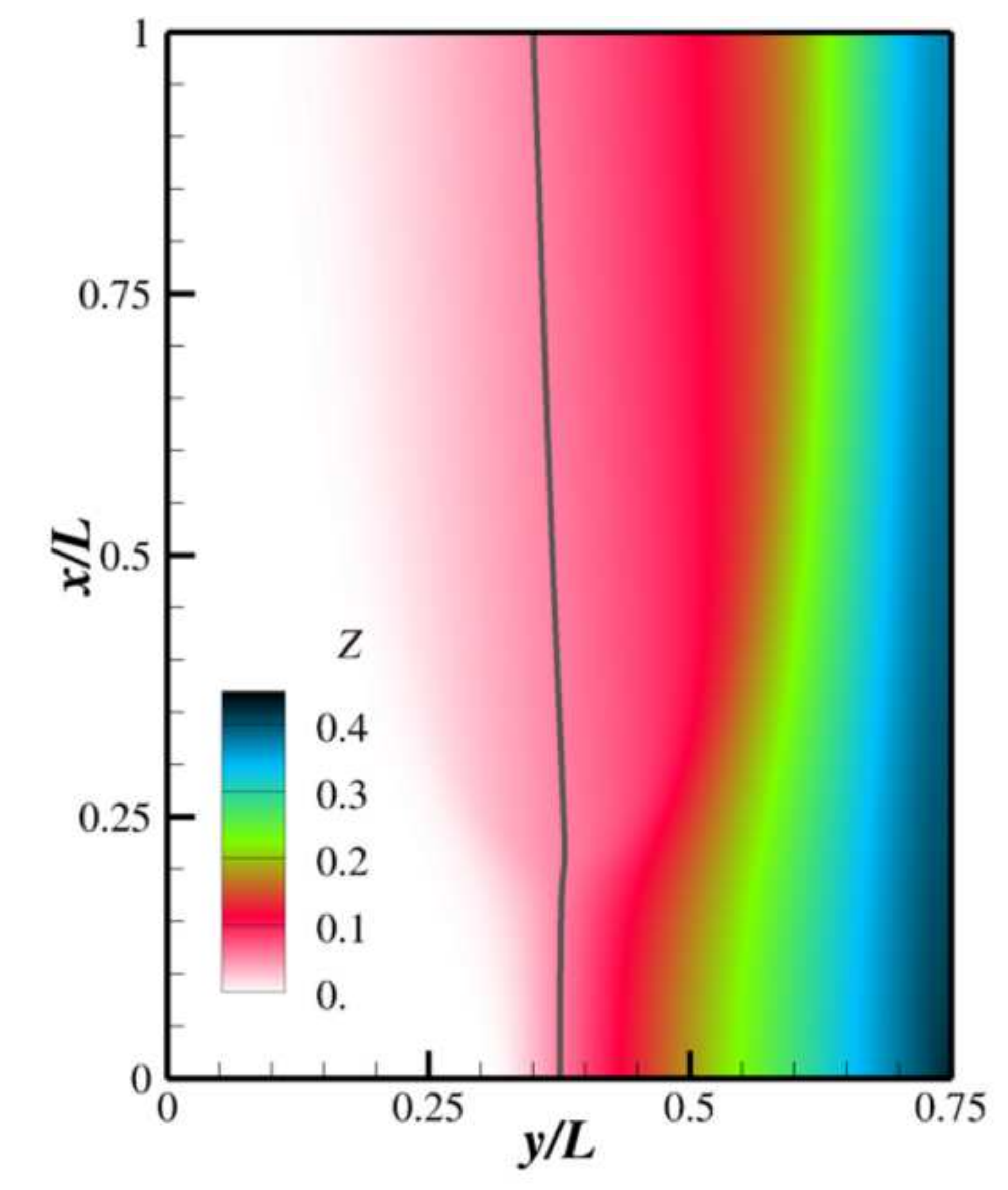

(

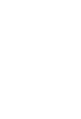

.

(1)



fam_big_z_m2_lamb_0p100.png

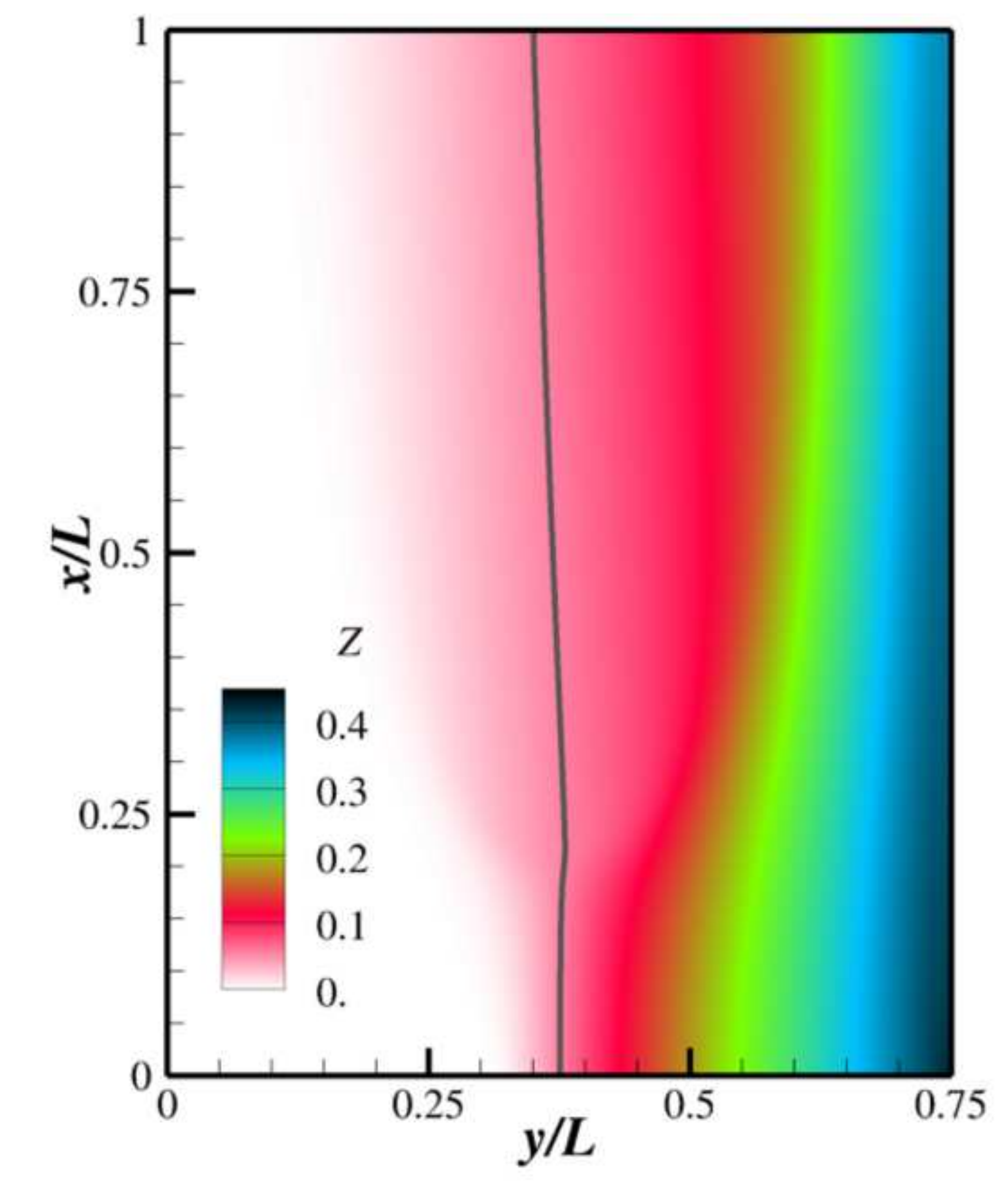

(

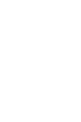

. 

fam_big_z_m2_lamb_0p200.png

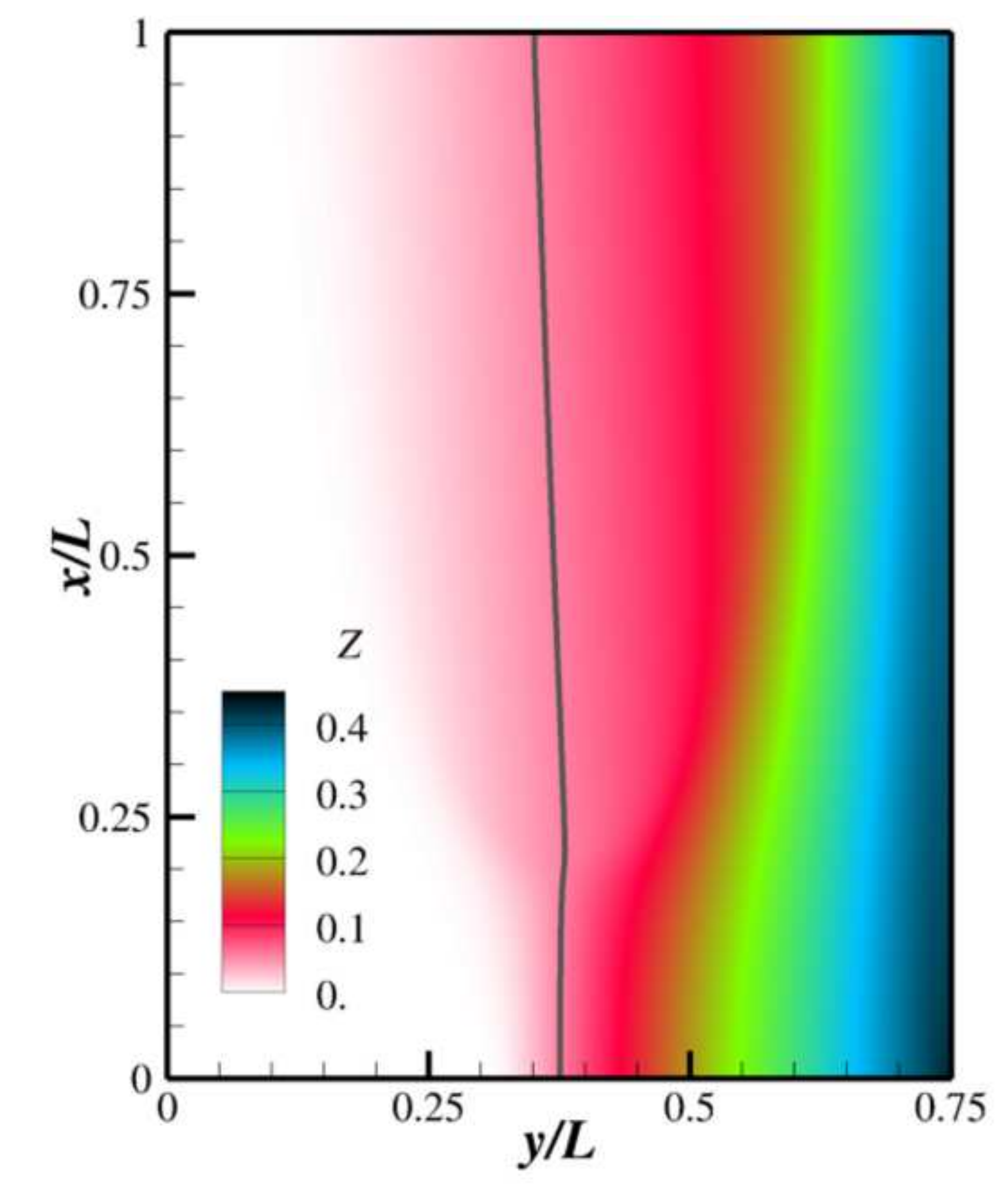

(

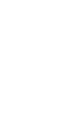

. 

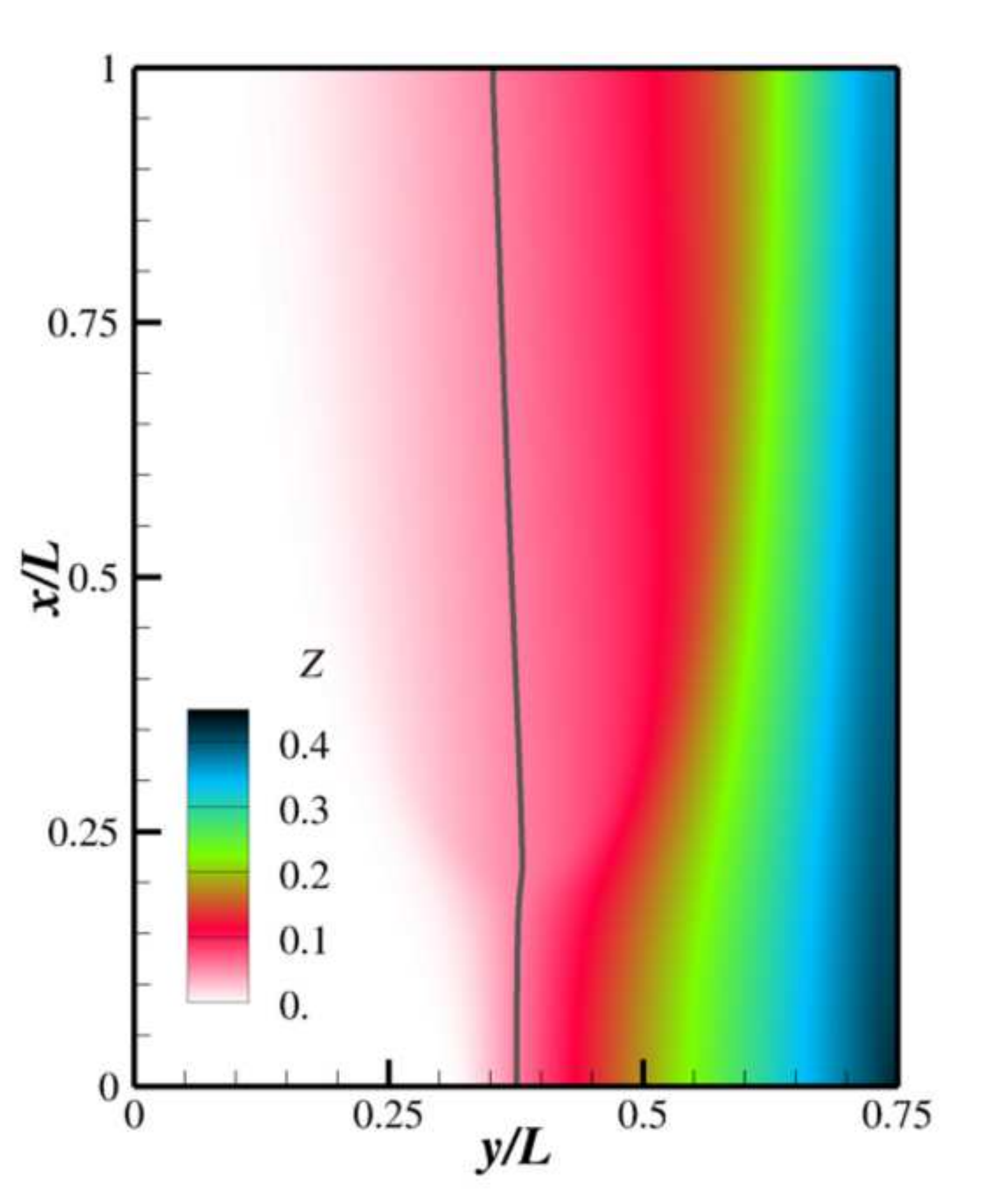
fam_big_z_q2_lamb_0p020.png

(n)

$\sqrt{2}$

(⿸丆口




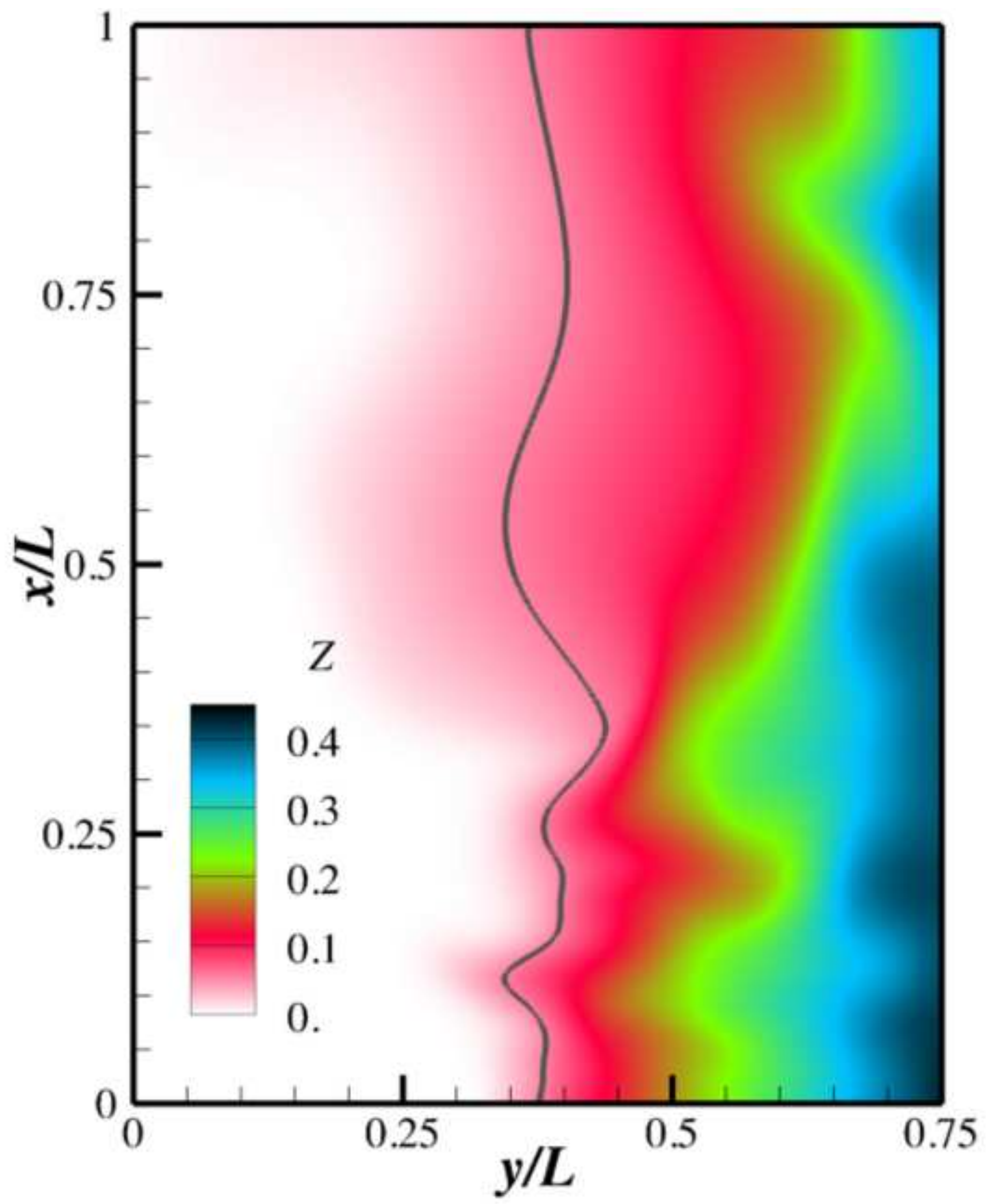




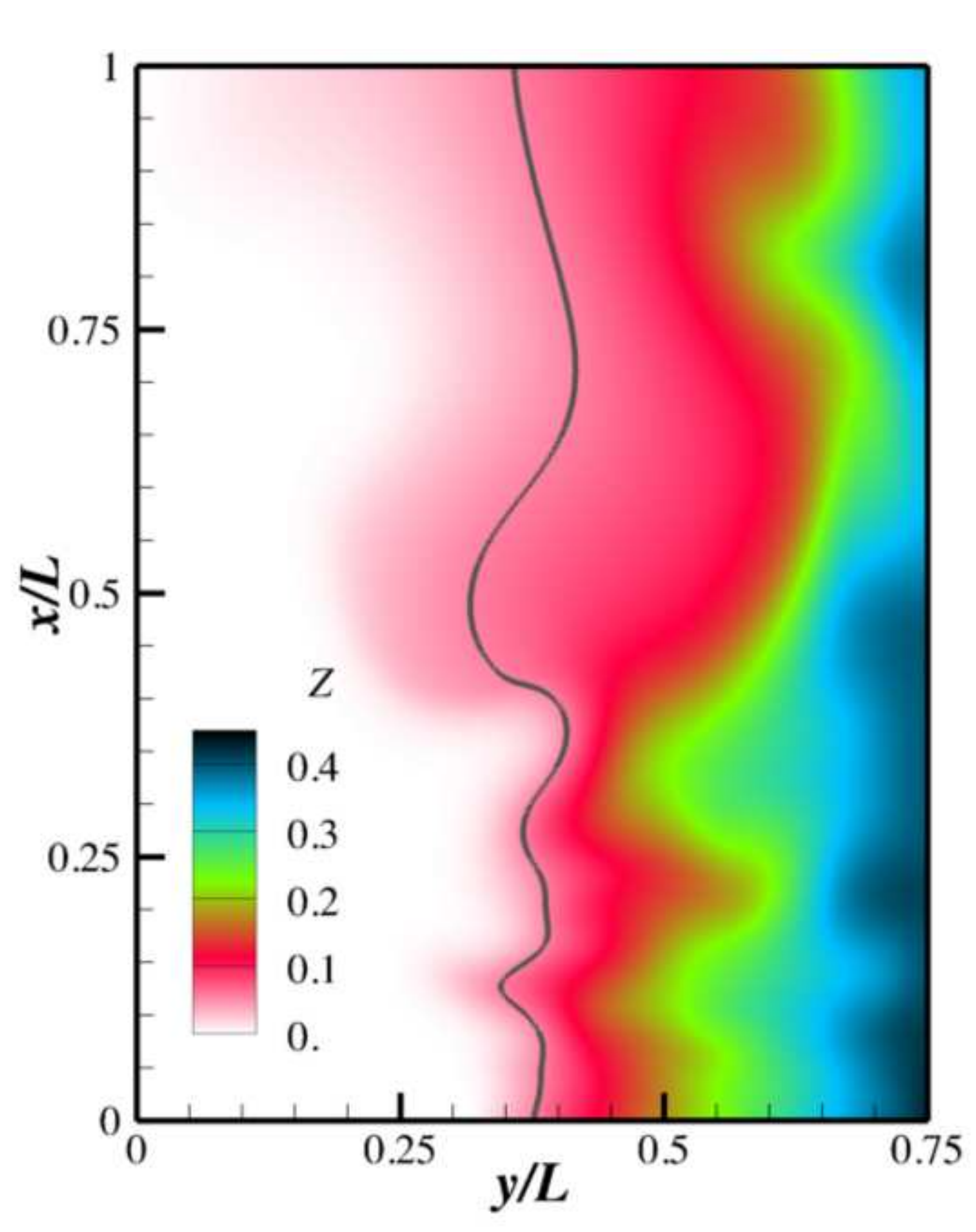




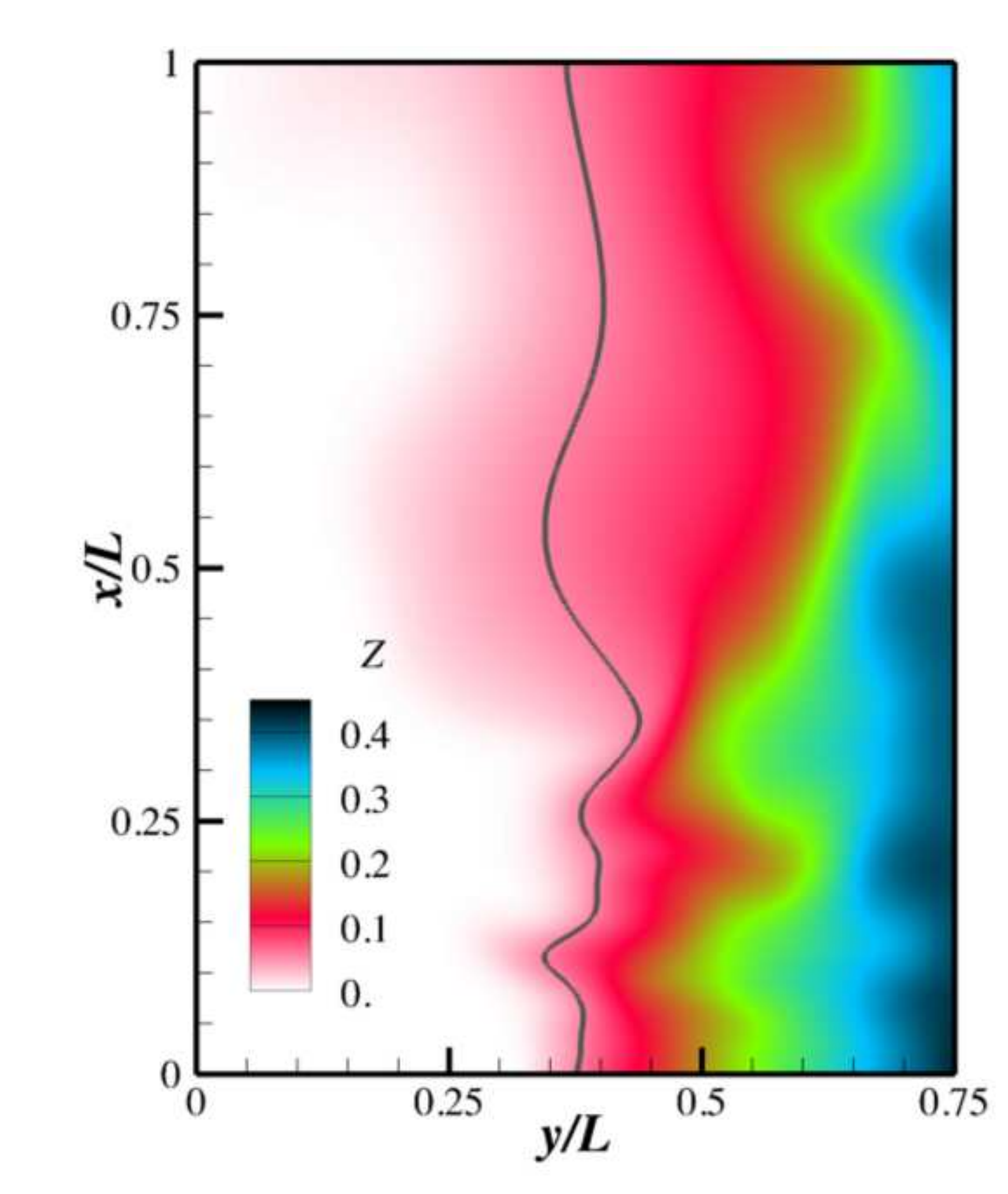

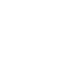

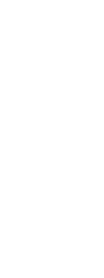




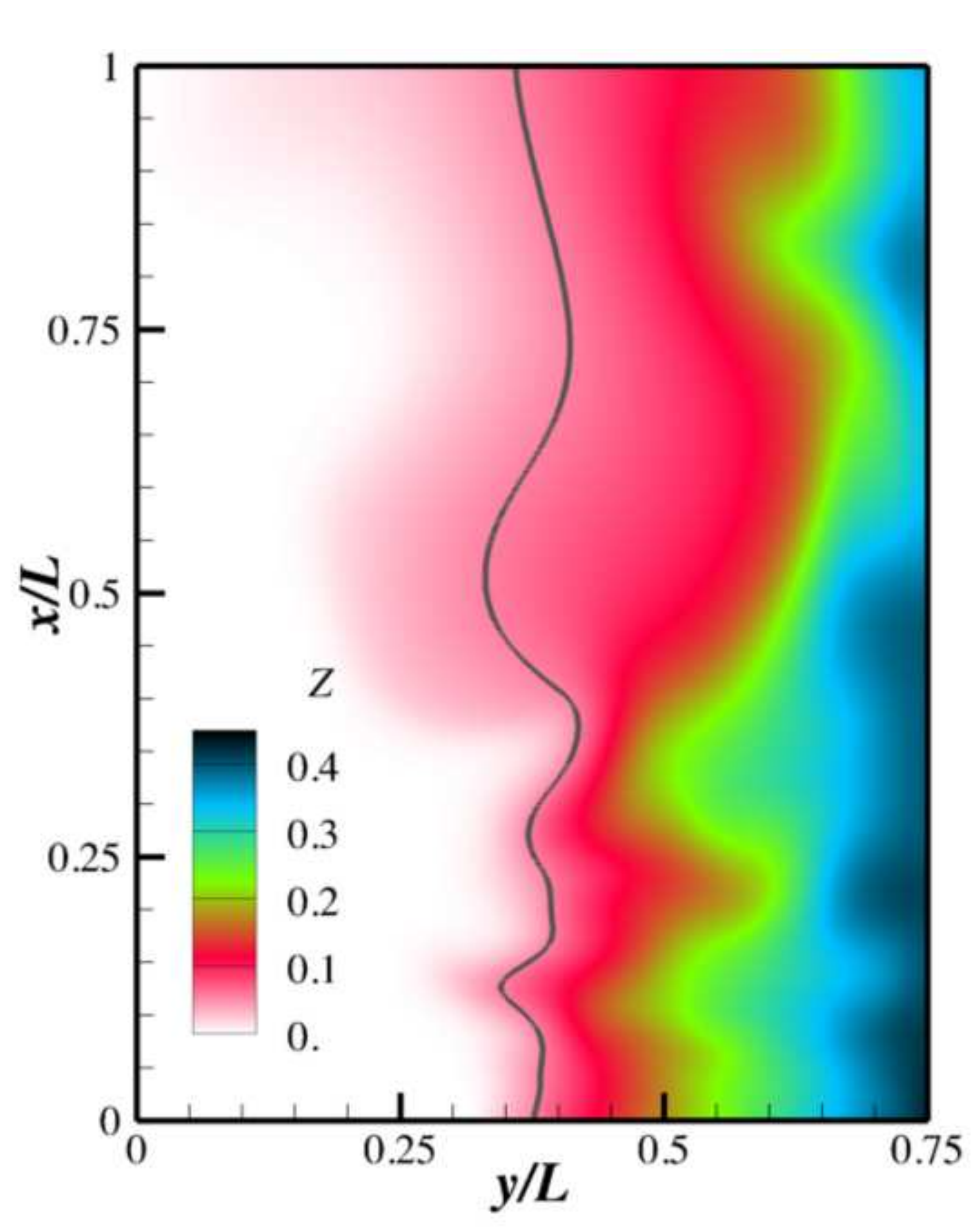

fam_big_z_trans_5p00.png

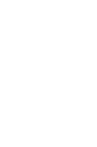

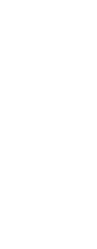


fam_cmask_m1_lamb_0p002.png

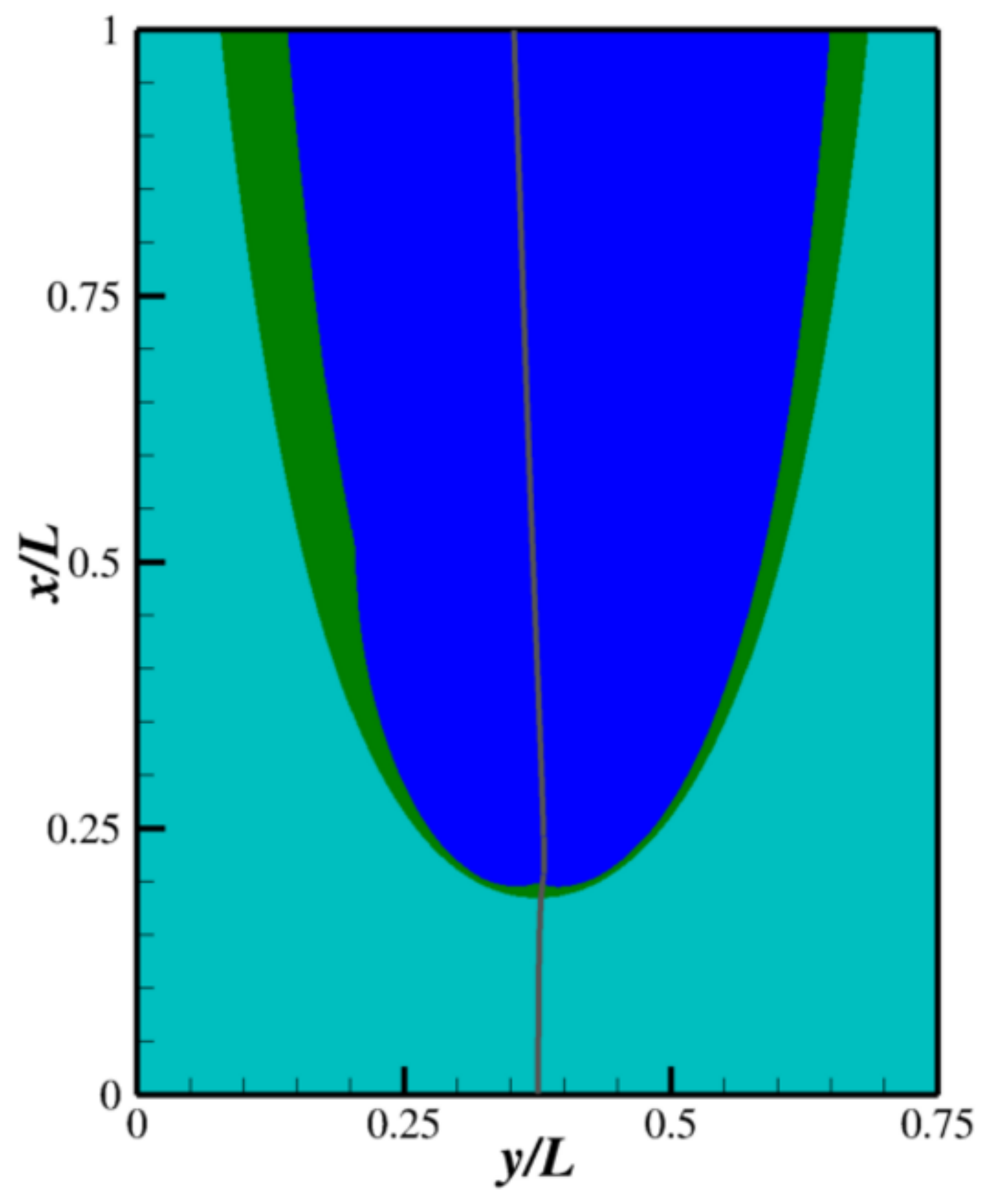

_crom_tamb_Op002png

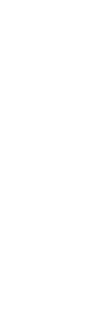


fam_cmask_m2_lamb_0p100.png

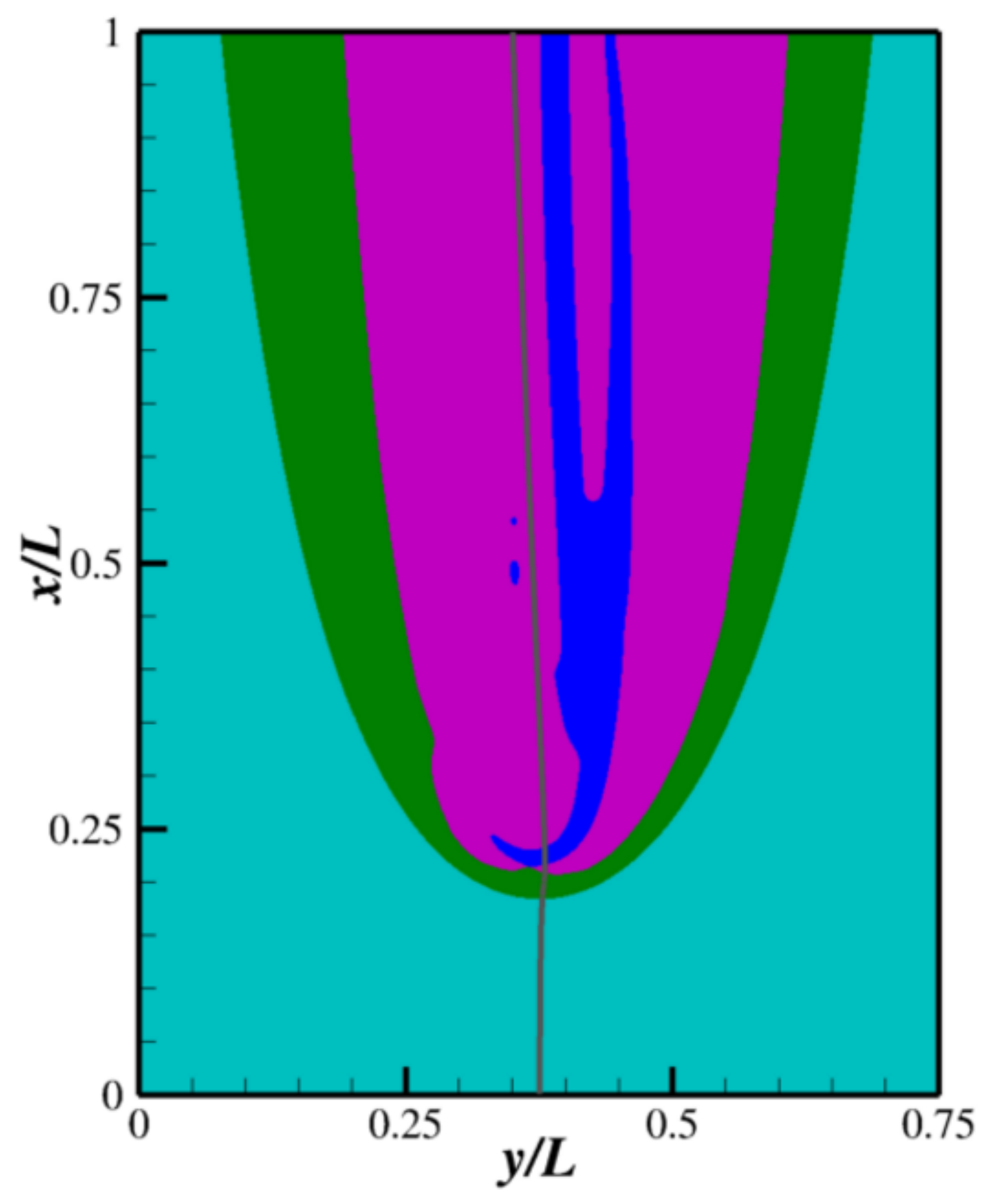

_am_cmask_m2_lamb_Op100.png

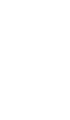


fam_m_radial1p2_co.pdf

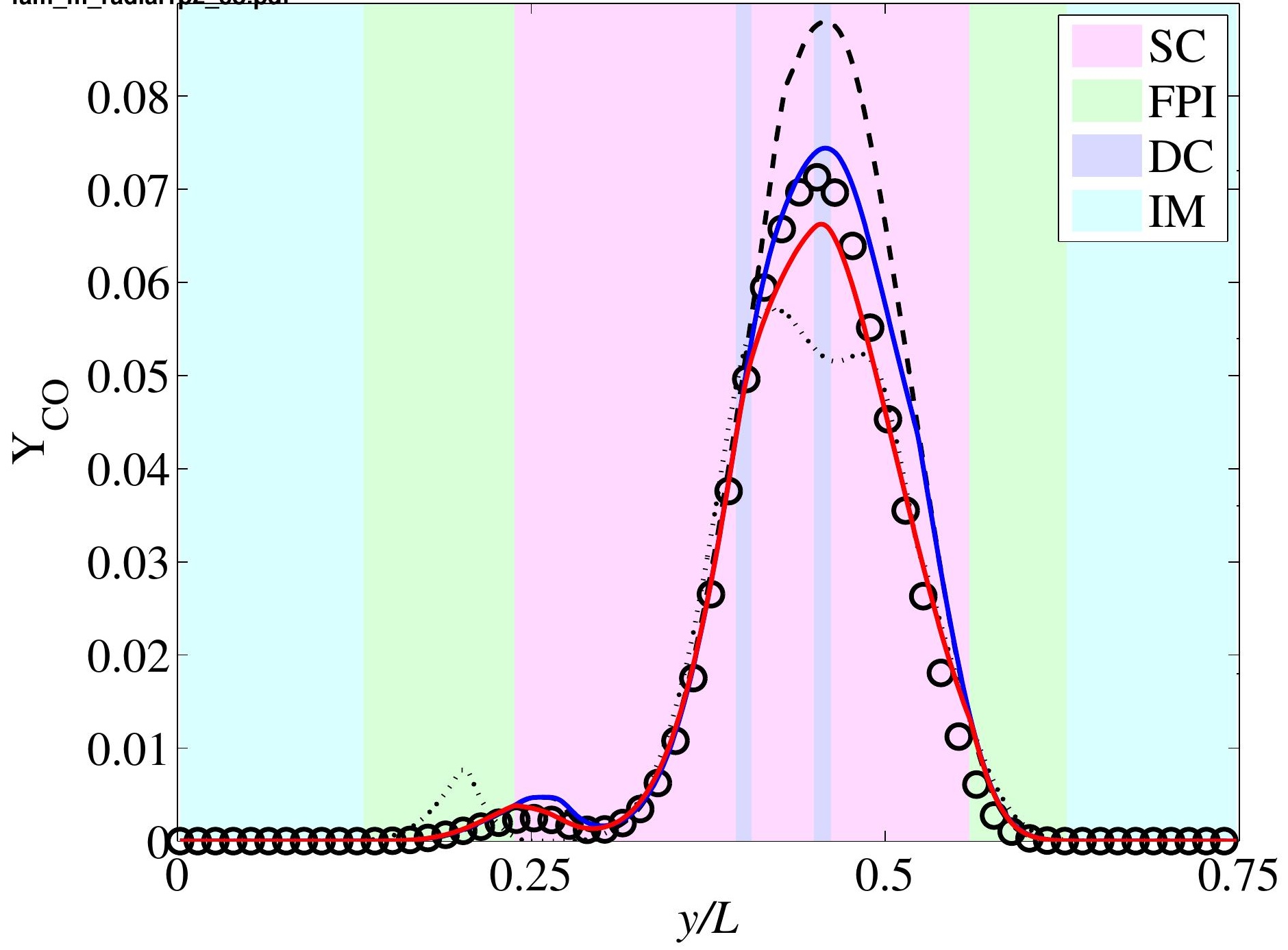


fam_m_radial1p2_=40.pdf

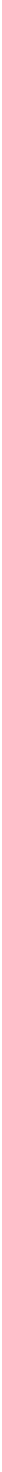




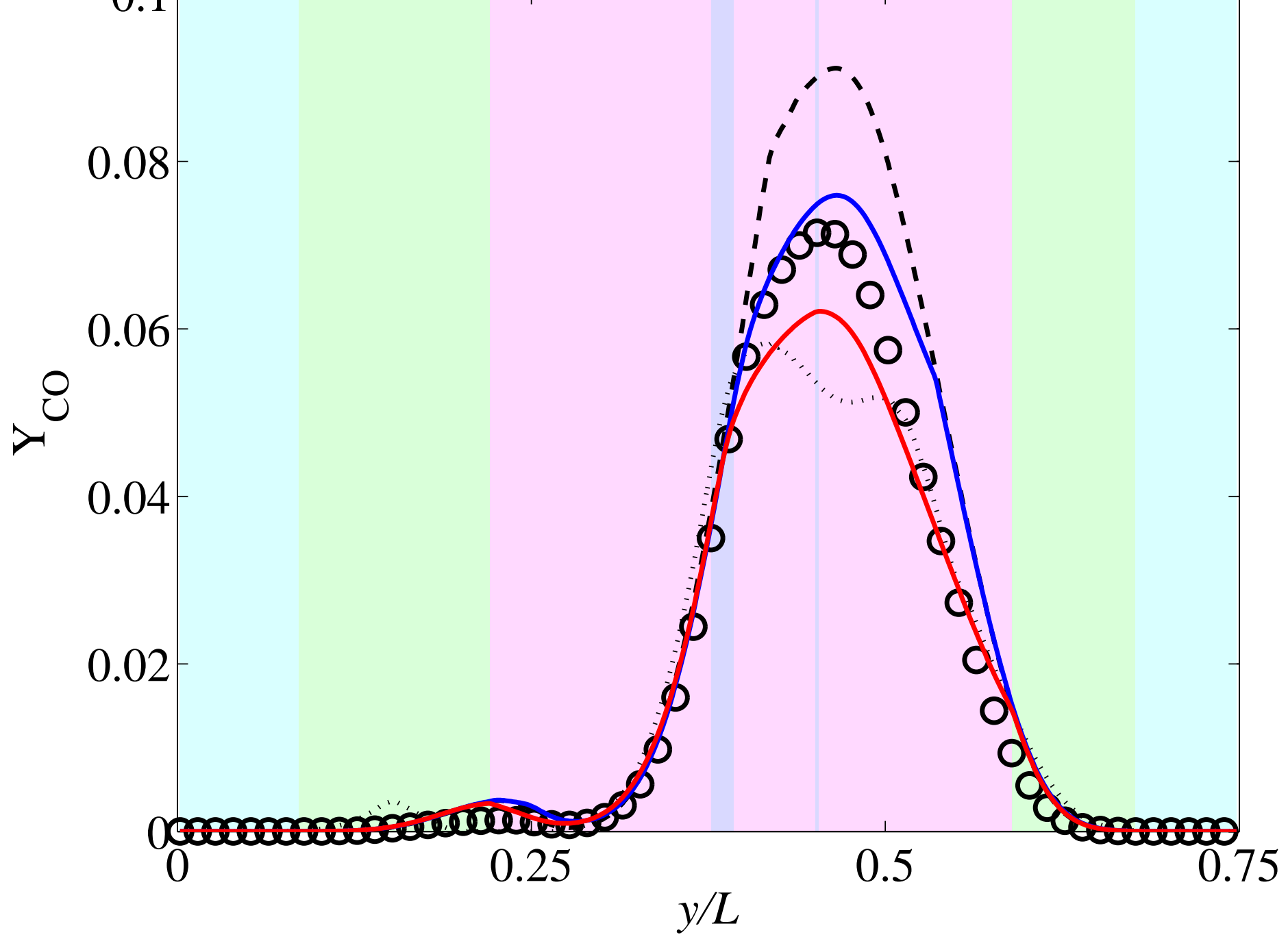

0.04

0.02

$y / L$ 
fam_m_radial1p8=q4o.pdf

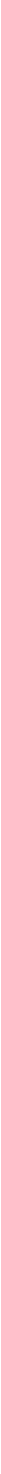




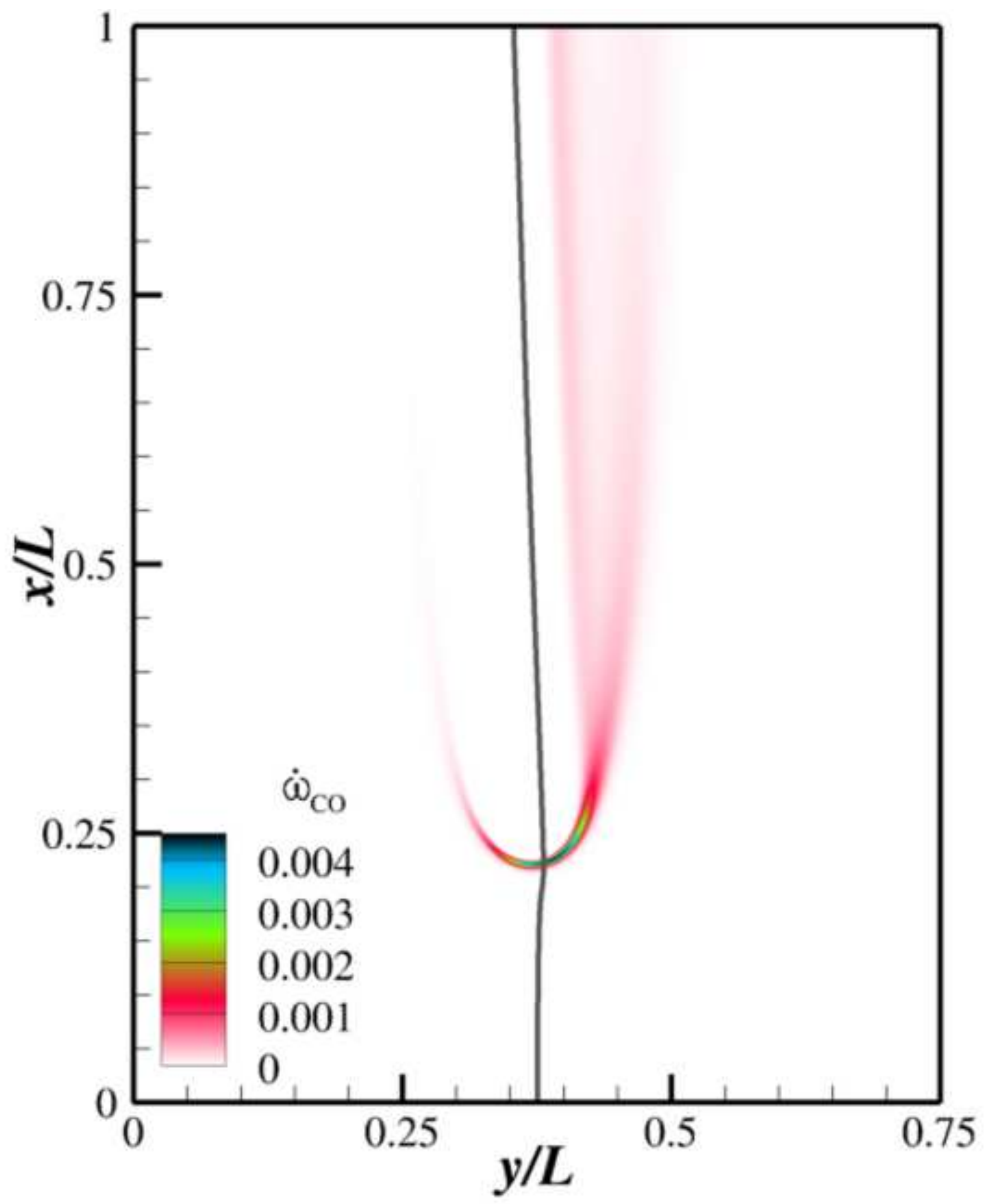




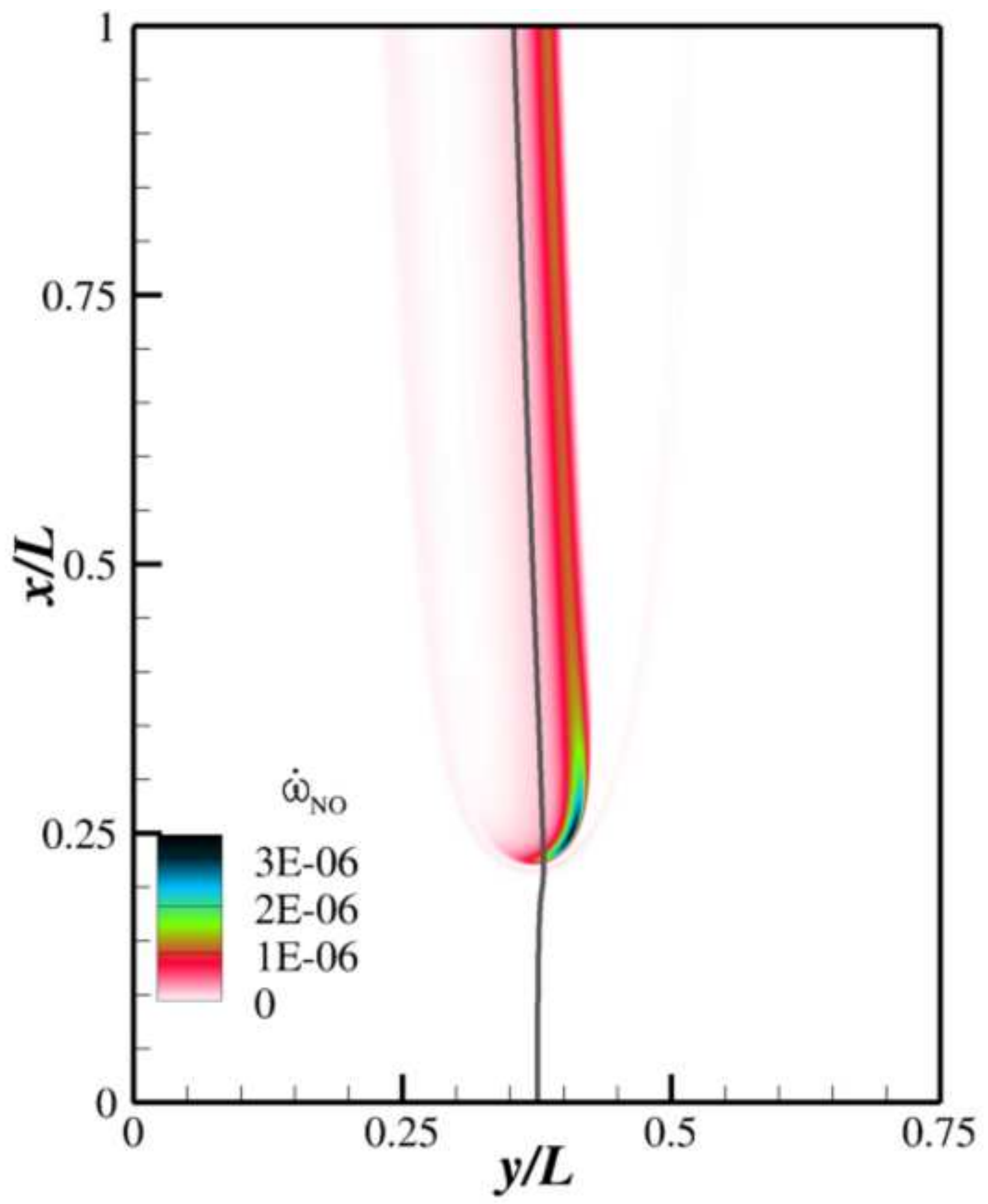




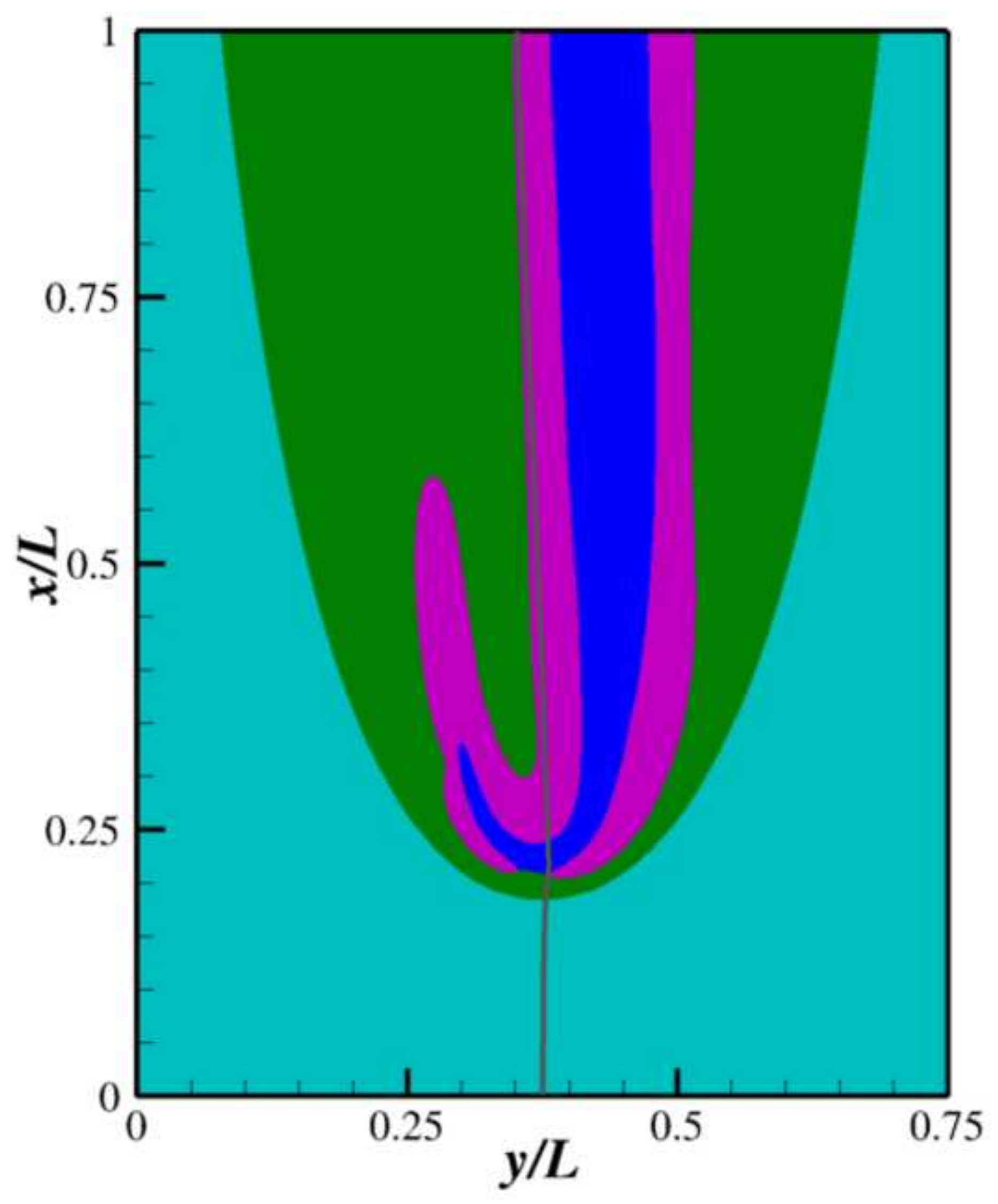




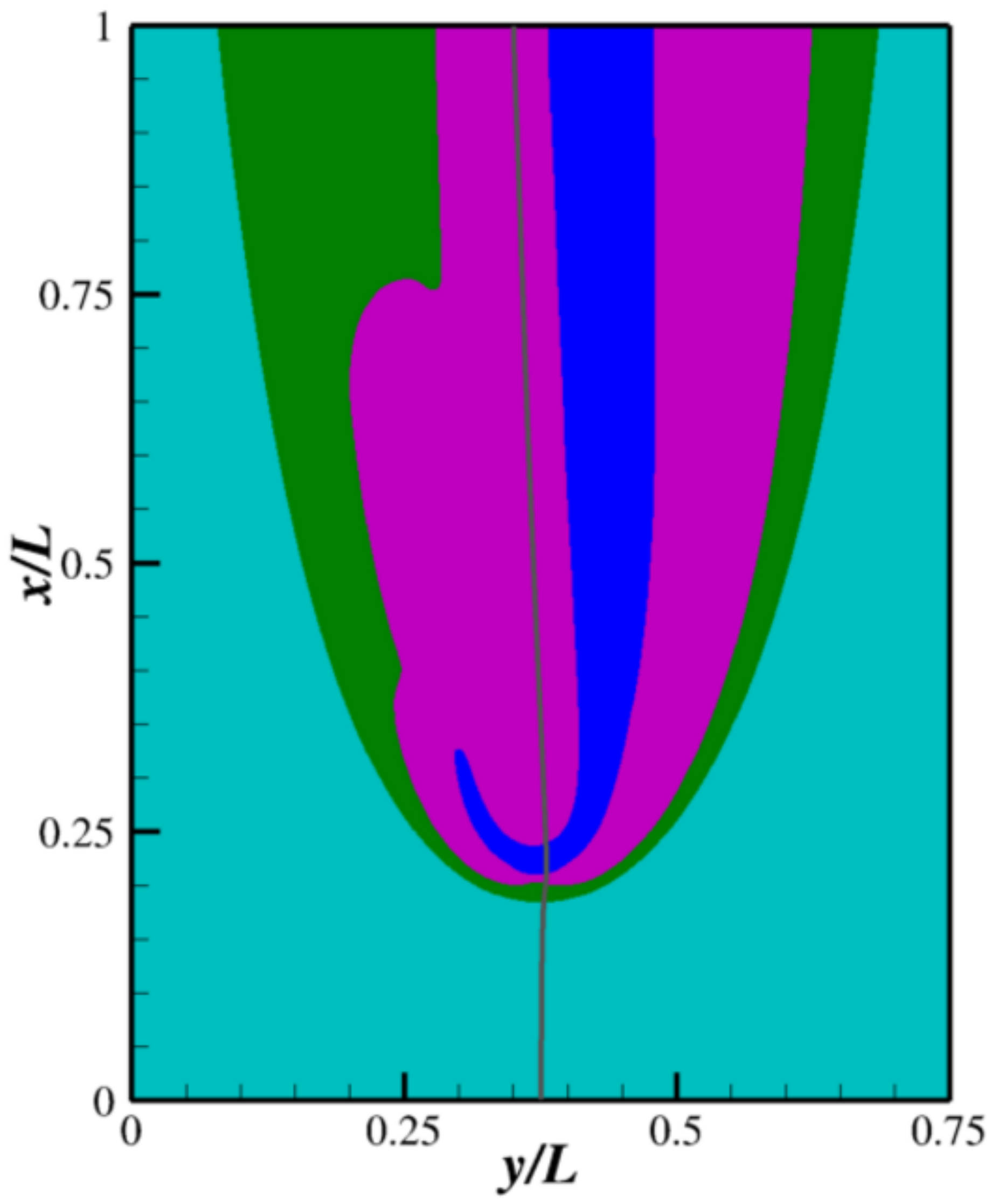




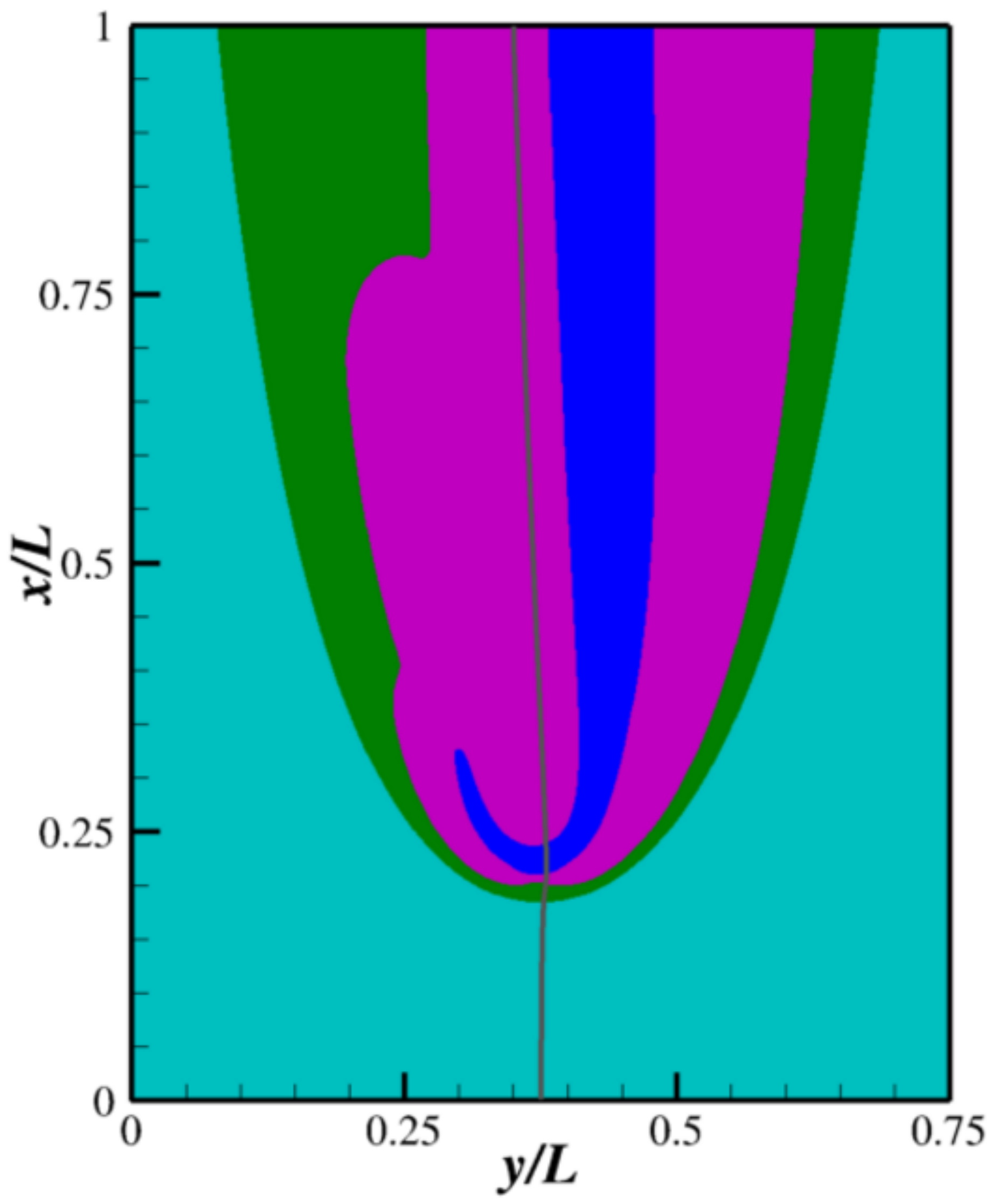




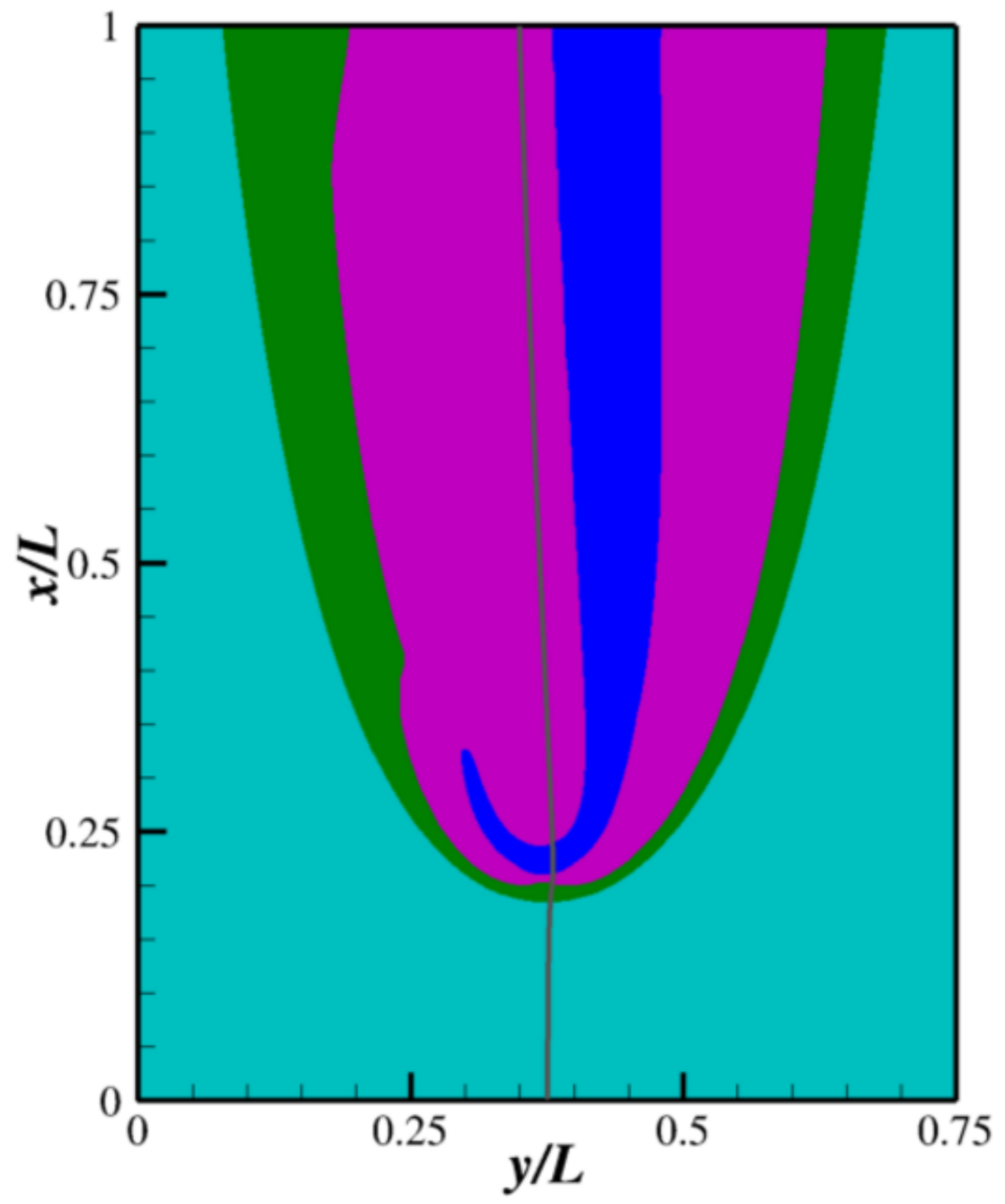




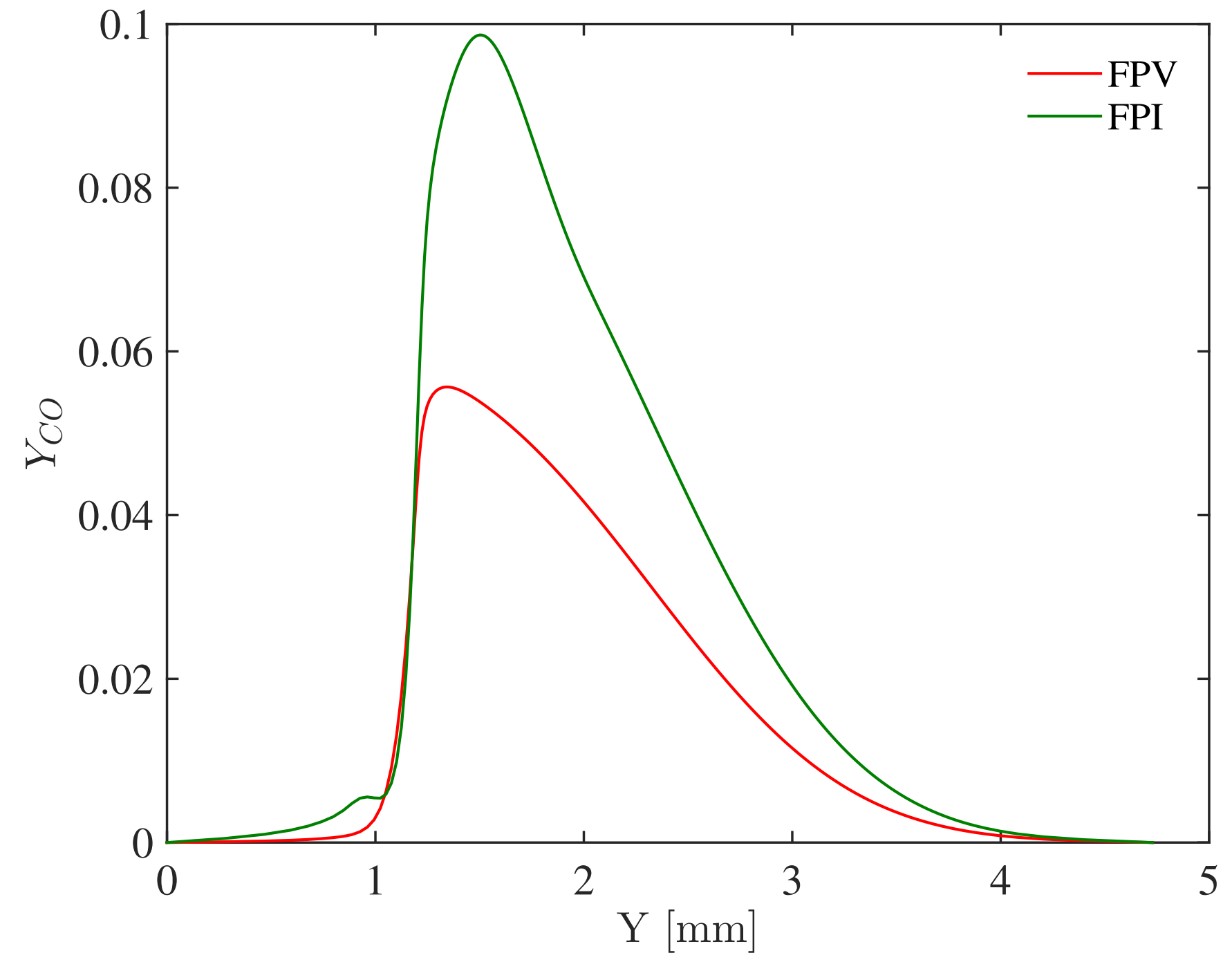

